\title{
2019 ESC Guidelines for the diagnosis and management of acute pulmonary embolism developed in collaboration with the European Respiratory Society (ERS)
}

The Task Force for the diagnosis and management of acute pulmonary embolism of the European Society of Cardiology (ESC)

Authors/Task Force Members: Stavros V. Konstantinides (Chairperson) (Germany/Greece), Guy Meyer (Co-Chairperson) (France), Cecilia Becattini (Italy), Héctor Bueno (Spain), Geert-Jan Geersing (Netherlands), Veli-Pekka Harjola (Finland), Menno V. Huisman (Netherlands), Marc Humbert (France), Catriona Sian Jennings (United Kingdom), David Jiménez (Spain), Nils Kucher (Switzerland), Irene Marthe Lang (Austria), Mareike Lankeit (Germany), Roberto Lorusso (Netherlands), Lucia Mazzolai (Switzerland), Nicolas Meneveau (France), Fionnuala Ní Áinle (Ireland), Paolo Prandoni (Italy), Piotr Pruszczyk (Poland), Marc Righini (Switzerland), Adam Torbicki (Poland), Eric Van Belle (France), and José Luis Zamorano (Spain)

@ERSpublications

New @ESCardio Guidelines for the Diagnosis and Management of Acute \#PulmonaryEmbolism developed in collaboration with @EuroRespSoc now available: \#cardiotwitter @erspublications http://bit.ly/2HnrJaj

Cite this article as: Konstantinides SV, Meyer G, Becattini C, et al. 2019 ESC Guidelines for the diagnosis and management of acute pulmonary embolism developed in collaboration with the European Respiratory Society (ERS). Eur Respir J 2019; 54: 1901647 [https://doi.org/10.1183/13993003.01647-2019].

Correspondence: Stavros V. Konstantinides, Center for Thrombosis and Hemostasis, Johannes Gutenberg University Mainz, Building 403, Langenbeckstr. 1, 55131 Mainz, Germany. E-mail: stavros.konstantinidesa unimedizin-mainz.de; and Department of Cardiology, Democritus University of Thrace, 68100 Alexandroupolis, Greece. E-mail: skonstamed.duth.gr.

Correspondence: Guy Meyer, Respiratory Medicine Department, Hôpital Européen Georges Pompidou, 20 Rue Leblanc, 75015 Paris, France. E-mail: guy.meyerdaphp.fr; and Université Paris Descartes, 15 rue de l'école de médecine 75006 Paris, France.

Supplementary data with additional supplementary tables complementing the full text, as well as section 11 on non-thrombotic PE, are available from erj.ersjournals.com

The disclosure forms of all experts involved in the development of these Guidelines are available on the ESC website (www.escardio.org/Guidelines/Clinical-Practice-Guidelines/Acute-Pulmonary-Embolism-Diagnosis-and-Management-of).

This publication in the European Respiratory Journal comprises a reprint of the official full text version of the article originally published in English in the European Heart Journal by Oxford University Press under licence from the European Society of Cardiology: Konstantinides SV, Meyer G, Becattini C, et al. 2019 ESC Guidelines for the diagnosis and management of acute pulmonary embolism developed in collaboration with the European Respiratory Society (ERS), Eur Heart $J$ doi/10.1093/eurheartj/ehz405. Reproduced by permission of Oxford University Press on behalf of the European Society of Cardiology. (C) European Society of Cardiology 2019 - All Rights Reserved. Please visit: https://www.escardio.org/Guidelines/Clinical-Practice-Guidelines/Acute-Pulmonary-Embolism-Diagnosis-and-Management-of; https://academic.oup.com/eurheartj 
Document Reviewers: Nazzareno Galié (CPG Review Coordinator) (Italy), J. Simon R. Gibbs (CPG Review Coordinator) (United Kingdom), Victor Aboyans (France), Walter Ageno (Italy), Stefan Agewall (Norway), Ana G. Almeida (Portugal), Felicita Andreotti (Italy), Emanuele Barbato (Italy), Johann Bauersachs (Germany), Andreas Baumbach (United Kingdom), Farzin Beygui (France), Jørn Carlsen (Denmark), Marco De Carlo (Italy), Marion Delcroix (Belgium), Victoria Delgado (Netherlands), Pilar Escribano Subias (Spain), Donna Fitzsimons (United Kingdom), Sean Gaine (Ireland), Samuel Z. Goldhaber (United States of America), Deepa Gopalan (United Kingdom), Gilbert Habib (France), Sigrun Halvorsen (Norway), David Jenkins (United Kingdom), Hugo A. Katus (Germany), Barbro Kjellström (Sweden), Mitja Lainscak (Slovenia), Patrizio Lancellotti (Belgium), Geraldine Lee (United Kingdom), Grégoire Le Gal (Canada), Emmanuel Messas (France), Joao Morais (Portugal), Steffen E. Petersen (United Kingdom), Anna Sonia Petronio (Italy), Massimo Francesco Piepoli (Italy), Susanna Price (United Kingdom), Marco Roffi (Switzerland), Aldo Salvi (Italy), Olivier Sanchez (France), Evgeny Shlyakhto (Russian Federation), lain A. Simpson (United Kingdom), Stefan Stortecky (Switzerland), Matthias Thielmann (Germany), Anton Vonk Noordegraaf (Netherlands).

\section{Preamble}

Guidelines summarize and evaluate available evidence with the aim of assisting health professionals in proposing the best management strategies for an individual patient with a given condition. Guidelines and their recommendations should facilitate decision making of health professionals in their daily practice. However, the final decisions concerning an individual patient must be made by the responsible health professional(s) in consultation with the patient and caregiver as appropriate.

A great number of guidelines have been issued in recent years by the European Society of Cardiology (ESC), as well as by other societies and organisations. Because of their impact on clinical practice, quality criteria for the development of guidelines have been established in order to make all decisions transparent to the user. The recommendations for formulating and issuing ESC Guidelines can be found on the ESC website (www.escardio.org/Guidelines-\&-Education/Clinical-Practice-Guidelines/Guidelines-development/ Writing-ESC-Guidelines). The ESC Guidelines represent the official position of the ESC on a given topic and are regularly updated.

Author/Task Force Member Affiliations, ESC Committee for Practice Guidelines (CPG) members and National Cardiac Societies document reviewers are listed in the Appendix.

M. Humbert, M. Delcroix, S. Gaine, O. Sanchez and A. Vonk Noordegraaf are Task Force Members representing the ERS.

ESC entities having participated in the development of this document are as follows. Associations: Acute Cardiovascular Care Association (ACCA), Association of Cardiovascular Nursing \& Allied Professions (ACNAP), European Association of Cardiovascular Imaging (EACVI), European Association of Percutaneous Cardiovascular Interventions (EAPCI), Heart Failure Association (HFA). Councils: Council on Cardiovascular Primary Care. Working Groups: Aorta and Peripheral Vascular Diseases, Cardiovascular Surgery, Pulmonary Circulation and Right Ventricular Function, Thrombosis

This publication is for personal and educational use only. No commercial use is authorized. No part of the ESC Guidelines may be translated or reproduced in any form without written permission from the ESC. Permission can be obtained upon submission of a written request to Oxford University Press, the publisher of the European Heart Journal and the party authorized to handle such permissions on behalf of the ESC (journals.permissions@oxfordjournals.org).

Disclaimer: The ESC Guidelines represent the views of the ESC and were produced after careful consideration of the scientific and medical knowledge, and the evidence available at the time of their publication. The ESC is not responsible in the event of any contradiction, discrepancy, and/or ambiguity between the ESC Guidelines and any other official recommendations or guidelines issued by the relevant public health authorities, in particular in relation to good use of healthcare or therapeutic strategies. Health professionals are encouraged to take the ESC Guidelines fully into account when exercising their clinical judgment, as well as in the determination and the implementation of preventive, diagnostic, or therapeutic medical strategies; however, the ESC Guidelines do not override, in any way whatsoever, the individual responsibility of health professionals to make appropriate and accurate decisions in consideration of each patient's health condition and in consultation with that patient and, where appropriate and/or necessary, the patient's caregiver. Nor do the ESC Guidelines exempt health professionals from taking into full and careful consideration the relevant official updated recommendations or guidelines issued by the competent public health authorities, in order to manage each patient's case in light of the scientifically accepted data pursuant to their respective ethical and professional obligations. It is also the health professional's responsibility to verify the applicable rules and regulations relating to drugs and medical devices at the time of prescription. 


\section{BOX 1 Abbreviations and acronyms}

AcT

AFE

ALT

AMPLIFY

ASPIRE

AV

b.i.d.

BNP

BP

BPA

b.p.m.

$\mathrm{Cl}$

$\mathrm{CO}$

CPET

$C P G$

$\mathrm{CrCl}$

CRNM

CT

CTED

CTEPH

CTPA

CUS

CYP3A4

DAMOVES

DASH

DVT

ECMO

ELISA

EMA

ERS

ESC

FAST

FDA

GUSTO

HAS-BLED

HERDOO2

H-FABP

HIV

HR

INR

IU

i.v.

IVC

LA

LMWH

LV

MRA

NCT

NOACls)

NT-proBNP

NYHA

OBRI

o.d.

OR

PAH

PAP

PE

PEA

PEITHO

PERC

PERT
Right ventricular outflow Doppler acceleration time

Amniotic fluid embolism

Alanine aminotransferase

Apixaban for the Initial Management of Pulmonary Embolism and Deep-Vein

Thrombosis as First-line Therapy

Aspirin to Prevent Recurrent Venous Thromboembolism trial

Arteriovenous

Bis in die (twice a day)

B-type natriuretic peptide

Blood pressure

Balloon pulmonary angioplasty

Beats per minute

Confidence interval

Cardiac output

Cardiopulmonary exercise testing

Committee for Practice Guidelines

Creatinine clearance

Clinically relevant non-major (bleeding)

Computed tomogram/tomographic/tomography

Chronic thromboembolic disease

Chronic thromboembolic pulmonary hypertension

Computed tomography pulmonary angiography/angiogram

Compression ultrasonography

Cytochrome 3A4

D-dimer, Age, Mutation, Obesity, Varicose veins, Eight [coagulation factor VIII], Sex

D-dimer, Age, Sex, Hormonal therapy

Deep vein thrombosis

Extracorporeal membrane oxygenation

Enzyme-linked immunosorbent assay

European Medicines Agency

European Respiratory Society

European Society of Cardiology

H-FABP, Syncope, Tachycardia (prognostic score)

US Food and Drug Administration

Global Utilization of Streptokinase and Tissue Plasminogen Activator for Occluded

Coronary Arteries

Hypertension, Abnormal renal/Liver function, Stroke, Bleeding history or predisposition,

Labile international normalized ratio, Elderly (>65 years), Drugs/alcohol concomitantly

Hyperpigmentation, Edema, or Redness in either leg; D-dimer level $\geqslant 250 \mu \mathrm{g} / \mathrm{L}$; Obesity with body mass index $\geqslant 30 \mathrm{~kg} / \mathrm{m}^{2}$; or Older age, $\geqslant 65$ years

Heart-type fatty acid-binding protein

Human immunodeficiency virus

Hazard ratio

International normalized ratio

International units

Intravenous

Inferior vena cava

Left atrium

Low-molecular weight heparin(s)

Left ventricle/ventricular

Magnetic resonance angiography

National clinical trial

Non-vitamin $\mathrm{K}$ antagonist oral anticoagulant(s)

$\mathrm{N}$-terminal pro B-type natriuretic peptide

New York Heart Association

Outpatient Bleeding Risk Index

Omni die (once a day)

Odds ratio

Pulmonary arterial hypertension

Pulmonary artery pressure

Pulmonary embolism

Pulmonary endarterectomy

Pulmonary Embolism Thrombolysis trial

Pulmonary Embolism Rule-out Criteria

Pulmonary Embolism Response Team 


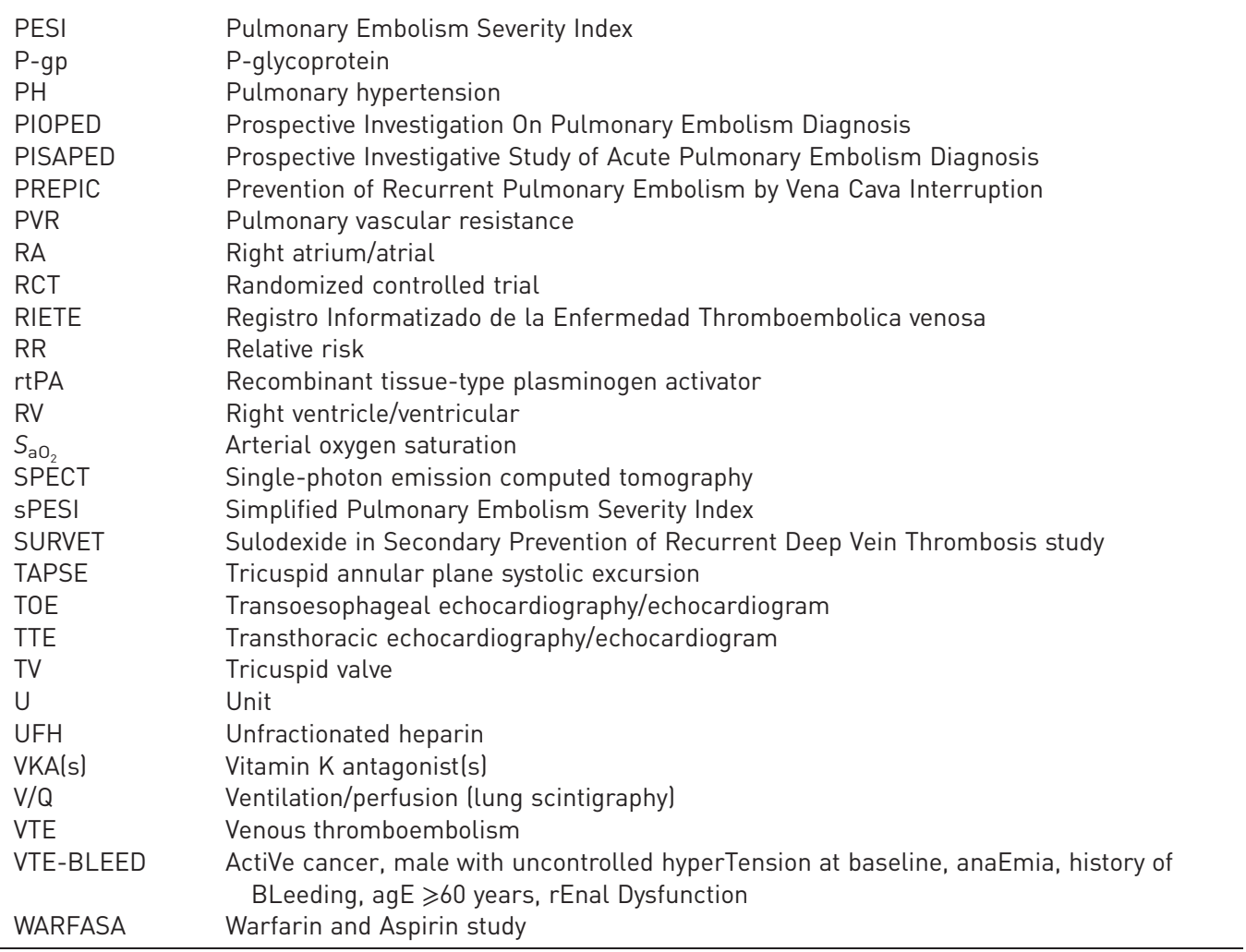

The ESC carries out a number of registries which are essential to assess, diagnostic/therapeutic processes, use of resources and adherence to Guidelines. These registries aim at providing a better understanding of medical practice in Europe and around the world, based on data collected during routine clinical practice.

The guidelines are developed together with derivative educational material addressing the cultural and professional needs for cardiologists and allied professionals. Collecting high-quality observational data, at appropriate time interval following the release of ESC Guidelines, will help evaluate the level of implementation of the Guidelines, checking in priority the key end points defined with the ESC Guidelines and Education Committees and Task Force members in charge.

The Members of this Task Force were selected by the ESC, including representation from its relevant ESC sub-specialty groups, in order to represent professionals involved with the medical care of patients with this pathology. Selected experts in the field undertook a comprehensive review of the published evidence for management of a given condition according to ESC Committee for Practice Guidelines (CPG) policy. A critical evaluation of diagnostic and therapeutic procedures was performed, including assessment of the risk-benefit ratio. The level of evidence and the strength of the recommendation of particular management options were weighed and graded according to predefined scales, as outlined in tables 1 and 2 .

The experts of the writing and reviewing panels provided declaration of interest forms for all relationships that might be perceived as real or potential sources of conflicts of interest. These forms were compiled into one file and can be found on the ESC website (www.escardio.org/guidelines). Any changes in declarations of interest that arise during the writing period were notified to the ESC and updated. The Task Force received its entire financial support from the ESC without any involvement from the healthcare industry.

The ESC CPG supervises and coordinates the preparation of new Guidelines. The Committee is also responsible for the endorsement process of these Guidelines. The ESC Guidelines undergo extensive review by the CPG and external experts. After appropriate revisions the Guidelines are approved by all the experts involved in the Task Force. The finalized document is approved by the CPG for publication in the European Heart Journal. The Guidelines were developed after careful consideration of the scientific and medical knowledge and the evidence available at the time of their dating.

The task of developing ESC Guidelines also includes the creation of educational tools and implementation programmes for the recommendations including condensed pocket guideline versions, summary slides, 


\begin{tabular}{|c|c|c|}
\hline Recommendation & Definition & Wording to use \\
\hline Class I & $\begin{array}{l}\text { Evidence and/or general agreement that a } \\
\text { given treatment or procedure is beneficial, } \\
\text { useful, effective. }\end{array}$ & Is recommended or is indicated \\
\hline Class II & \multicolumn{2}{|c|}{$\begin{array}{l}\text { Conflicting evidence and/or a divergence of opinion about the usefulness/efficacy of } \\
\text { the given treatment or procedure }\end{array}$} \\
\hline Class Ila & $\begin{array}{l}\text { Weight of evidence/opinion is in } \\
\text { favour of usefulness/efficacy. }\end{array}$ & Should be considered \\
\hline Class IIb & $\begin{array}{l}\text { Usefulness/efficacy is less well } \\
\text { established by evidence/opinion. }\end{array}$ & May be considered \\
\hline Class III & $\begin{array}{l}\text { Evidence or general agreement that the } \\
\text { given treatment or procedure is not } \\
\text { useful/effective, and in some cases may be } \\
\text { harmful. }\end{array}$ & Is not recommended \\
\hline
\end{tabular}

booklets with essential messages, summary cards for non-specialists and an electronic version for digital applications (smartphones, etc.). These versions are abridged and thus, for more detailed information, the user should always access to the full text version of the Guidelines, which is freely available via the ESC website and hosted on the EHJ website. The National Societies of the ESC are encouraged to endorse, translate and implement all ESC Guidelines. Implementation programmes are needed because it has been shown that the outcome of disease may be favourably influenced by the thorough application of clinical recommendations.

Health professionals are encouraged to take the ESC Guidelines fully into account when exercising their clinical judgment, as well as in the determination and the implementation of preventive, diagnostic or therapeutic medical strategies. However, the ESC Guidelines do not override in any way whatsoever the individual responsibility of health professionals to make appropriate and accurate decisions in consideration of each patient's health condition and in consultation with that patient or the patient's caregiver where appropriate and/or necessary. It is also the health professional's responsibility to verify the rules and regulations applicable in each country to drugs and devices at the time of prescription.

\section{Introduction}

2.1. Why do we need new Guidelines on the diagnosis and management of pulmonary embolism? This document follows the previous ESC Guidelines focusing on the clinical management of pulmonary embolism (PE), published in 2000, 2008, and 2014. Many recommendations have been retained or their validity has been reinforced; however, new data have extended or modified our knowledge in respect of the optimal diagnosis, assessment, and treatment of patients with PE. These new aspects have been integrated into previous knowledge to suggest optimal and-whenever possible-objectively validated management strategies for patients with suspected or confirmed PE. To limit the length of the article text, additional information, tables, figures, and references are available as supplementary data.

These Guidelines focus on the diagnosis and management of acute PE in adult patients. For further details specifically related to the diagnosis and management of deep vein thrombosis (DVT), the reader is referred to the joint consensus document of the ESC Working Groups of Aorta and Peripheral Vascular Diseases, and Pulmonary Circulation and Right Ventricular Function [1].

\section{TABLE 2 Levels of evidence}

Level of

evidence $A$

Level of

evidence B

Level of

evidence C
Data derived from multiple randomized clinical traits or meta-analyses

Data derived from a single randomized clinical trial or large non-randomized studies

Consensus of opinion of the experts and/or small studies, retrospective studies, registries 
2.2. What is new in the 2019 Guidelines?

\subsubsection{New/revised concepts in 2019}

\section{Diagnosis}

D-dimer cut-off values adjusted for age or clinical probability can be used as an alternative to the fixed cut-off value.

Updated information is provided on the radiation dosage when using CTPA and a lung scan to diagnose PE (table 6).

\section{Risk assessment}

A clear definition of haemodynamic instability and high-risk PE is provided (table 4).

Assessment of PE severity and early PE-related risk is recommended, in addition to comorbidity/ aggravating conditions and overall death risk.

A clear word of caution that RV dysfunction may be present, and affect early outcomes, in patients at "low risk" based on clinical risk scores.

\section{Treatment in the acute phase}

Thoroughly revised section on haemodynamic and respiratory support for high-risk PE (Section 6.1). A dedicated management algorithm is proposed for high-risk PE (supplementary figure 1).

NOACs are recommended as the first choice for anticoagulation treatment in a patient eligible for NOACs; VKAs are an alternative to NOACs.

The risk-adjusted management algorithm (figure 6) was revised to take into consideration clinical PE severity, aggravating conditions/comorbidity, and the presence of RV dysfunction.

\section{Chronic treatment after the first 3 months}

Risk factors for VTE recurrence have been classified according to high, intermediate, or low recurrence risk (table 11).

Potential indications for extended anticoagulation are discussed, including the presence of a minor transient or reversible risk factor for the index $\mathrm{PE}$, any persisting risk factor, or no identifiable risk factor. Terminology such as "provoked" versus "unprovoked" PE/VTE is no longer supported by the Guidelines, as it is potentially misleading and not helpful for decision-making regarding the duration of anticoagulation. VTE recurrence scores are presented and discussed in parallel with bleeding scores for patients on anticoagulation treatment (supplementary tables 13 and 14 respectively).

A reduced dose of apixaban or rivaroxaban for extended anticoagulation should be considered after the first 6 months of treatment.

\section{PE in cancer}

Edoxaban or rivaroxaban should be considered as an alternative to LMWH, with a word of caution for patients with gastrointestinal cancer due to the increased bleeding risk with NOACs.

\section{PE in pregnancy}

A dedicated diagnostic algorithm is proposed for suspected PE in pregnancy (figure 7).

Updated information is provided on radiation absorption related to procedures used for diagnosing PE in pregnancy (table 12).

\section{Long-term sequelae}

An integrated model of patient care after PE is proposed to ensure optimal transition from hospital to community care.

Recommendations on patient care have been extended to the entire spectrum of post-PE symptoms and functional limitation, not only CTEPH.

A new comprehensive algorithm is proposed for patient follow-up after acute PE (figure 8).

CTEPH: chronic thromboembolic pulmonary hypertension; CTPA: computed tomography pulmonary angiography; LMWH: low-molecular weight heparin; NOAC(s): non-vitamin $\mathrm{K}$ antagonist oral anticoagulant(s); PE: pulmonary embolism; RV: right ventricular; VKA(s): vitamin K antagonist(s); VTE: venous thromboembolism.

\subsubsection{Changes in recommendations between 2014 and 2019}

\section{Recommendations}

Rescue thrombolytic therapy is recommended for patients who deteriorate haemodynamically.

Surgical embolectomy or catheter-directed treatment should be considered as alternatives to rescue thrombolytic therapy for patients who deteriorate haemodynamically.

D-dimer measurement and clinical prediction rules should be considered to rule out PE during pregnancy or the post-partum period.

Further evaluation may be considered for asymptomatic PE survivors at increased risk for CTEPH.

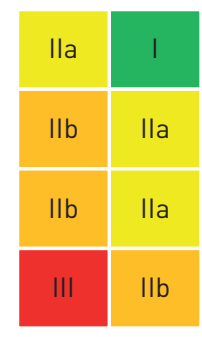

CTEPH: chronic thromboembolic pulmonary hypertension; PE: pulmonary embolism. Coloured columns indicate classes of recommendation (see table 1 for colour coding). 


\subsubsection{Main new recommendations 2019}

\section{Diagnosis}

A D-dimer test, using an age-adjusted cut-off or adapted to clinical probability, should be considered as an alternative to the fixed cut-off level.

If a positive proximal CUS is used to confirm PE, risk assessment should be considered to guide management.

V/Q SPECT may be considered for PE diagnosis.

\section{Risk assessment}

Assessment of the RV by imaging or laboratory biomarkers should be considered, even in the presence of a low PESI or a SPESI of 0.

Validated scores combining clinical, imaging, and laboratory prognostic factors may be considered to further stratify PE severity.

\section{Treatment in the acute phase}

When oral anticoagulation is initiated in a patient with PE who is eligible for a NOAC lapixaban, dabigatran, edoxaban, or rivaroxaban), a NOAC is the recommended form of anticoagulant treatment.

Set-up of multidisciplinary teams for management of high-risk and selected cases of intermediate-risk PE should be considered, depending on the resources and expertise available in each hospital.

ECMO may be considered, in combination with surgical embolectomy or catheter-directed treatment, in refractory circulatory collapse or cardiac arrest.

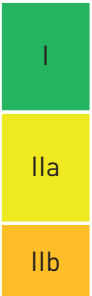

\section{Chronic treatment and prevention of recurrence}

Indefinite treatment with a VKA is recommended for patients with antiphospholipid antibody syndrome.

Extended anticoagulation should be considered for patients with no identifiable risk factor for the index PE event.

Extended anticoagulation should be considered for patients with a persistent risk factor other than antiphospholipid antibody syndrome.

Extended anticoagulation should be considered for patients with a minor transient/reversible risk factor for the index PE event.

A reduced dose of apixaban or rivaroxaban should be considered after the first 6 months.

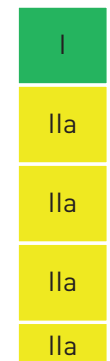

\section{$\mathrm{PE}$ in cancer}

Edoxaban or rivaroxaban should be considered as an alternative to LMWH, with the exception of patients with gastrointestinal cancer.

\section{$P E$ in pregnancy}

Amniotic fluid embolism should be considered in a pregnant or post-partum woman, with unexplained haemodynamic instability or respiratory deterioration, and disseminated intravascular coagulation.

Thrombolysis or surgical embolectomy should be considered for pregnant women with high-risk PE. NOACs are not recommended during pregnancy or lactation.

\section{Post-PE care and long-term sequelae}

Routine clinical evaluation is recommended 3-6 months after acute PE.

An integrated model of care is recommended after acute PE to ensure optimal transition from hospital to ambulatory care.

It is recommended that symptomatic patients with mismatched perfusion defects on a V/Q scan $>3$ months after acute PE are referred to a pulmonary hypertension/CTEPH expert centre, taking into account the results of echocardiography, natriuretic peptide, and/or cardiopulmonary exercise testing.

CPET: cardiopulmonary exercise testing; $\mathrm{CTEPH}$ : chronic thromboembolic pulmonary hypertension; CUS compression ultrasonography; ECMO: extracorporeal membrane oxygenation; LMWH: low-molecular weight heparin; NOAC(s): non-vitamin K antagonist oral anticoagulant(s); PE: pulmonary embolism; PESI: Pulmonary Embolism Severity Index; RV: right ventricular; SPECT: single-photon emission computed tomography; SPESI: simplified Pulmonary Embolism Severity Index; VKA(s): vitamin K antagonist(s); V/Q: ventilation/perfusion (lung scintigraphy). Coloured columns indicate classes of recommendation (see table 1 for colour coding). 


\section{General considerations}

\subsection{Epidemiology}

Venous thromboembolism (VTE), clinically presenting as DVT or PE, is globally the third most frequent acute cardiovascular syndrome behind myocardial infarction and stroke [2]. In epidemiological studies, annual incidence rates for PE range from 39 to 115 per 100000 population; for DVT, incidence rates range from 53 to 162 per 100000 population [3,4]. Cross-sectional data show that the incidence of VTE is almost eight times higher in individuals aged $\geqslant 80$ years than in the fifth decade of life [3]. In parallel, longitudinal studies have revealed a rising tendency in annual PE incidence rates [4-7] over time. Together with the substantial hospital-associated, preventable, and indirect annual expenditures for VTE (an estimated total of up to EUR 8.5 billion in the European Union) [8], these data demonstrate the importance of PE and DVT in ageing populations in Europe and other areas of the world. They further suggest that VTE will increasingly pose a burden on health systems worldwide in the years to come.

PE may cause $\leqslant 300000$ deaths per year in the US, ranking high among the causes of cardiovascular mortality [3]. In six European countries with a total population of 454.4 million, more than 370000 deaths were related to VTE in 2004, as estimated on the basis of an epidemiological model [9]. Of these patients, $34 \%$ died suddenly or within a few hours of the acute event, before therapy could be initiated or take effect. Of the other patients, death resulted from acute PE that was diagnosed after death in $59 \%$ and only $7 \%$ of patients who died early were correctly diagnosed with PE before death [9].

Time trend analyses in European, Asian, and North American populations suggest that case fatality rates of acute PE may be decreasing $[4-7,10,11]$. Increased use of more effective therapies and interventions, and possibly better adherence to guidelines $[12,13]$, has most likely exerted a significant positive effect on the prognosis of $\mathrm{PE}$ in recent years. However, there is also a tendency towards overdiagnosis of (subsegmental or even non-existent) PE in the modern era [14], and this might in turn lead to a false drop in case fatality rates by inflating the denominator, i.e. the total number of $\mathrm{PE}$ cases.

Figure 1 summarizes the existing data on global trends in PE, highlighting increasing incidence rates in parallel with decreasing case fatality rates over an $\sim 15$ year period.

In children, studies have reported an annual incidence of VTE of between 53 and 57 per 100000 among hospitalized patients $[19,20]$, and between 1.4 and 4.9 per 100000 in the community overall $[21,22]$.

\subsection{Predisposing factors}

There is an extensive collection of predisposing environmental and genetic factors for VTE; a list of predisposing (risk) factors is shown in table 3. VTE is considered to be a consequence of the interaction between patient-related-usually permanent-risk factors and setting-related-usually temporary-risk factors. Since categorization of temporary and permanent risk factors for VTE is important for assessing the risk of recurrence, and consequently for decision-making on chronic anticoagulation, it is discussed in more detail in section 8 of these Guidelines.

Major trauma, surgery, lower-limb fractures and joint replacements, and spinal cord injury are strong provoking factors for VTE $[23,24]$. Cancer is a well-recognized predisposing factor for VTE. The risk of VTE varies with different types of cancer [25, 26]; pancreatic cancer, haematological malignancies, lung cancer, gastric cancer, and brain cancer carry the highest risk [27, 28]. Moreover, cancer is a strong risk factor for all-cause mortality following an episode of VTE [29].

Oestrogen-containing oral contraceptive agents are associated with an elevated VTE risk, and contraceptive use is the most frequent VTE risk factor in women of reproductive age [30-32]. More specifically, combined oral contraceptives (containing both an oestrogen and a progestogen) are associated with an approximately two- to six-fold increase in VTE risk over baseline $[32,33]$. In general, the absolute VTE risk remains low in the majority of the $>100$ million combined oral contraceptive users worldwide [34]; however, VTE risk factors, including severe inherited thrombophilia (discussed in section 8) [35], increase this risk. Third-generation combined oral contraceptives, containing progestogens such as desogestrel or gestodene, are associated with a higher VTE risk than the second-generation combined oral contraceptives, which contain progestogens such as levonorgestrel or norgestrel [36, 37]. On the other hand, hormone-releasing intrauterine devices and some progesterone-only pills (used at contraceptive doses) are not associated with a significant increase in VTE risk $[33,38]$; consequently, and following counselling and full risk assessment, these options are often proposed to women with a personal or strong family history of VTE.

In post-menopausal women who receive hormone replacement therapy, the risk of VTE varies widely depending on the formulation used [39].

Infection is a common trigger for VTE [23, 40, 41]. Blood transfusion and erythropoiesis-stimulating agents are also associated with an increased risk of VTE [23, 42]. 

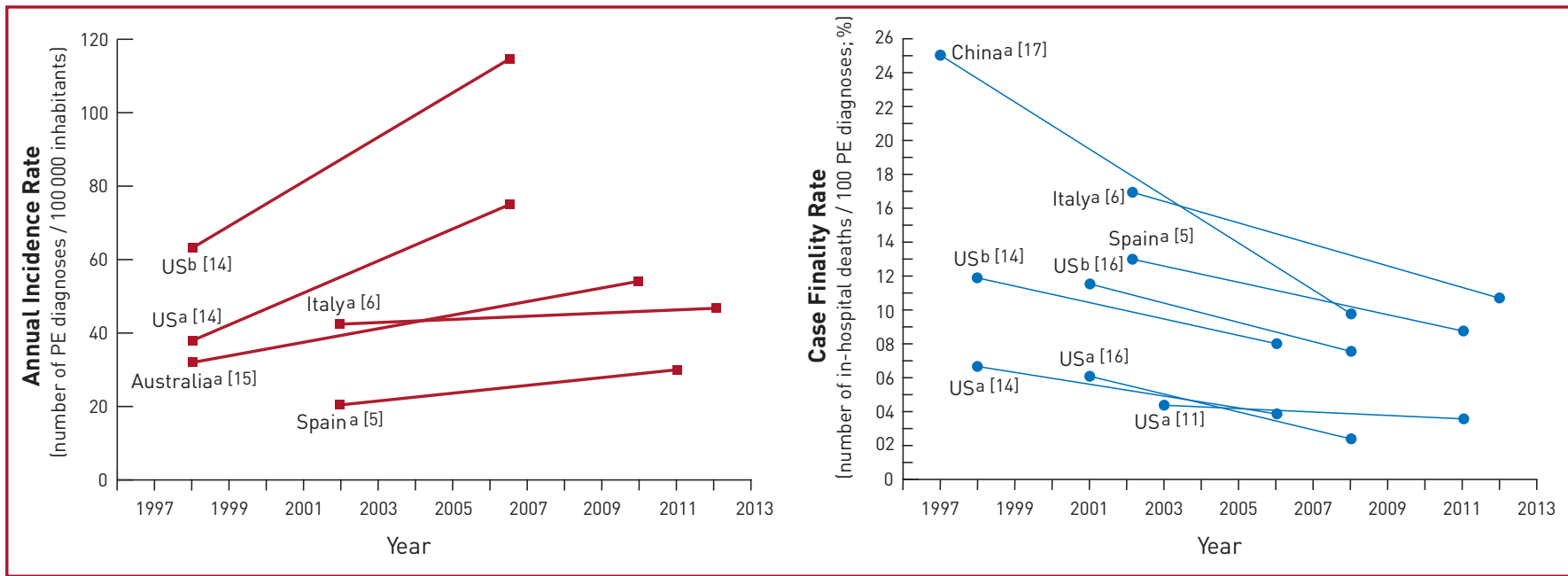

FIGURE 1 Trends in annual incidence rates (left panel) and case fatality rates (right panel) of pulmonary embolism around the world, based on data retrieved from various references [5, 6, 11, 14-17]. Reproduced with permission from [18]. PE: pulmonary embolism; US: United States. ${ }^{a}$ PE listed as principal diagnosis. ${ }^{\mathrm{b}}$ Any listed code for PE was considered.

In children, PE is usually associated with DVT and is rarely unprovoked. Serious chronic medical conditions and central venous lines are considered likely triggers of PE [43].

VTE may be viewed as part of the cardiovascular disease continuum, and common risk factors-such as cigarette smoking, obesity, hypercholesterolaemia, hypertension, and diabetes mellitus [44-47] —are shared with arterial disease, notably atherosclerosis [48-51]. However, this may be an indirect association mediated, at least in part, by the complications of coronary artery disease and, in the case of smoking, cancer [52, 53]. Myocardial infarction and heart failure increase the risk of PE [54, 55]. Conversely, patients with VTE have an increased risk of subsequent myocardial infarction and stroke, or peripheral arterial embolization [56].

\subsection{Pathophysiology and determinants of outcome}

Acute PE interferes with both circulation and gas exchange. Right ventricular (RV) failure due to acute pressure overload is considered the primary cause of death in severe PE. Pulmonary artery pressure (PAP) increases if $>30-50 \%$ of the total cross-sectional area of the pulmonary arterial bed is occluded by thromboemboli [57]. PE-induced vasoconstriction, mediated by the release of thromboxane A2 and serotonin, contributes to the initial increase in pulmonary vascular resistance (PVR) after PE [58]. Anatomical obstruction and hypoxic vasoconstriction in the affected lung area lead to an increase in PVR, and a proportional decrease in arterial compliance [59].

The abrupt increase in PVR results in RV dilation, which alters the contractile properties of the RV myocardium via the Frank-Starling mechanism. The increase in RV pressure and volume leads to an increase in wall tension and myocyte stretch. The contraction time of the RV is prolonged, while neurohumoral activation leads to inotropic and chronotropic stimulation. Together with systemic vasoconstriction, these compensatory mechanisms increase PAP, improving flow through the obstructed pulmonary vascular bed and thus temporarily stabilizing systemic blood pressure (BP). However, the extent of immediate adaptation is limited, as a non-preconditioned, thin-walled RV is unable to generate a mean $\mathrm{PAP}>40 \mathrm{mmHg}$.

Prolongation of RV contraction time into early diastole in the left ventricle (LV) leads to leftward bowing of the interventricular septum [60]. The desynchronization of the ventricles may be exacerbated by the development of right bundle branch block. As a result, LV filling is impeded in early diastole, and this may lead to a reduction in the cardiac output $(\mathrm{CO})$, and contribute to systemic hypotension and haemodynamic instability [61].

As described above, excessive neurohumoral activation in PE can be the result of both abnormal RV wall tension and circulatory shock. The finding of massive infiltrates of inflammatory cells in the RV myocardia of patients who died within $48 \mathrm{~h}$ of acute PE may be explained by high levels of epinephrine released as a result of the PE-induced "myocarditis" [62]. This inflammatory response might explain the secondary haemodynamic destabilization that sometimes occurs $24-48 \mathrm{~h}$ after acute PE, although early recurrence of $\mathrm{PE}$ may be an alternative explanation in some of these cases. 
TABLE 3 Predisposing factors for venous thromboembolism (data modified from Rogers et al. [23] and Anderson and Spencer [24])

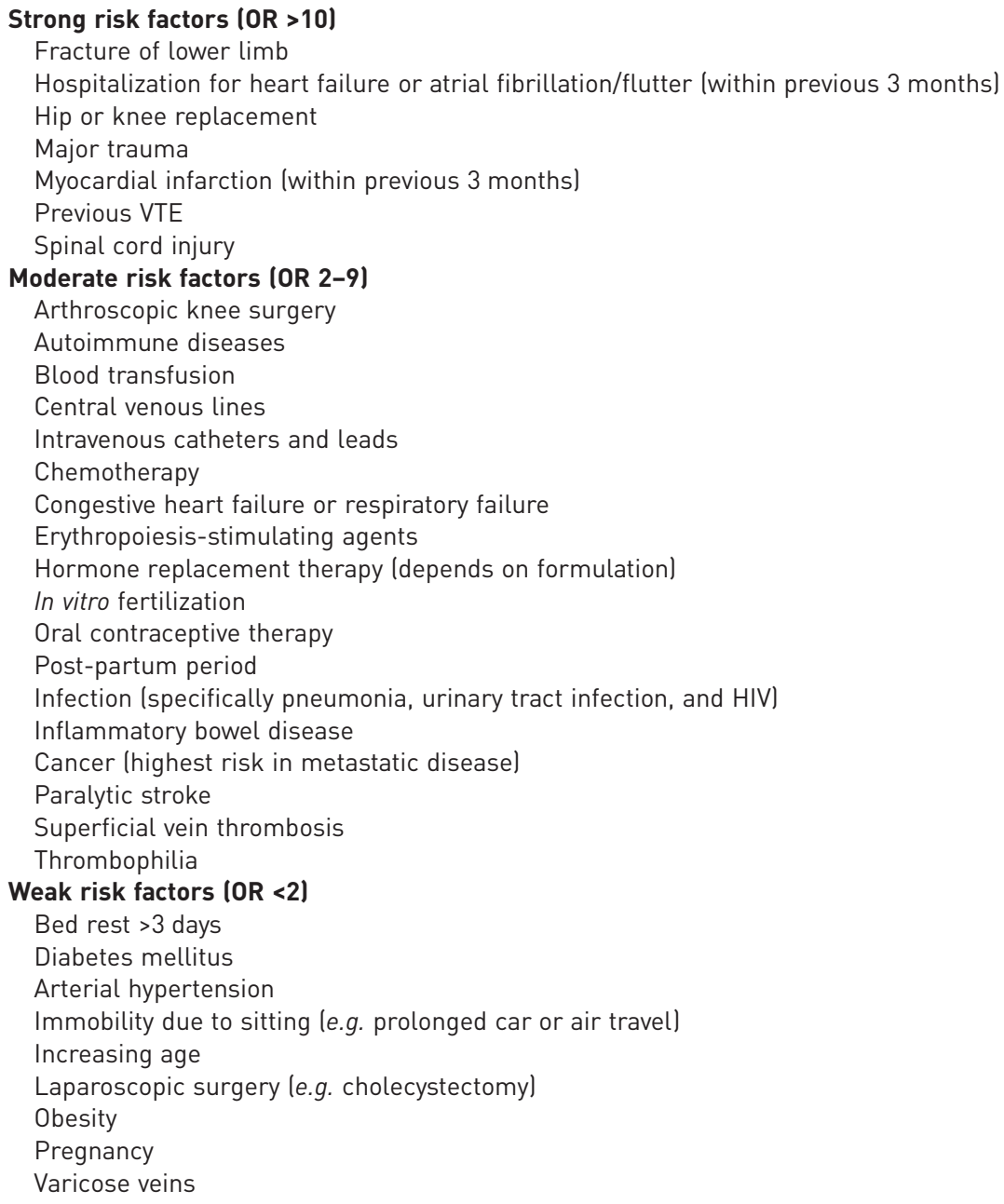

HIV: human immunodeficiency virus; OR: odds ratio; VTE: venous thromboembolism.

Finally, the association between elevated circulating levels of biomarkers of myocardial injury and an adverse early outcome indicates that RV ischaemia is of pathophysiological significance in the acute phase of PE [63, 64]. Although RV infarction is uncommon after PE, it is likely that the imbalance between oxygen supply and demand can result in damage to cardiomyocytes, and further reduce contractile forces. Systemic hypotension is a critical element in this process, leading to impairment of the coronary driving pressure to the overloaded RV.

The detrimental effects of acute PE on the RV myocardium and the circulation are summarized in Figure 2.

Respiratory failure in PE is predominantly a consequence of haemodynamic disturbances [66]. Low CO results in desaturation of the mixed venous blood. Zones of reduced flow in obstructed pulmonary arteries, combined with zones of overflow in the capillary bed served by non-obstructed pulmonary vessels, result in ventilation/perfusion mismatch, which contributes to hypoxaemia. In about one-third of patients, right-to-left shunting through a patent foramen ovale can be detected by echocardiography; this is caused by an inverted pressure gradient between the right atrium (RA) and left atrium, and may lead to severe hypoxaemia, and an increased risk of paradoxical embolization and stroke [67]. Finally, even if they do not affect haemodynamics, small distal emboli may create areas of alveolar haemorrhage resulting in haemoptysis, pleuritis, and pleural effusion, which is usually mild. This clinical presentation is known as "pulmonary infarction". Its effect on gas exchange is normally mild, except in patients with pre-existing cardiorespiratory disease.

In view of the above pathophysiological considerations, acute RV failure, defined as a rapidly progressive syndrome with systemic congestion resulting from impaired RV filling and/or reduced RV flow output [68], 


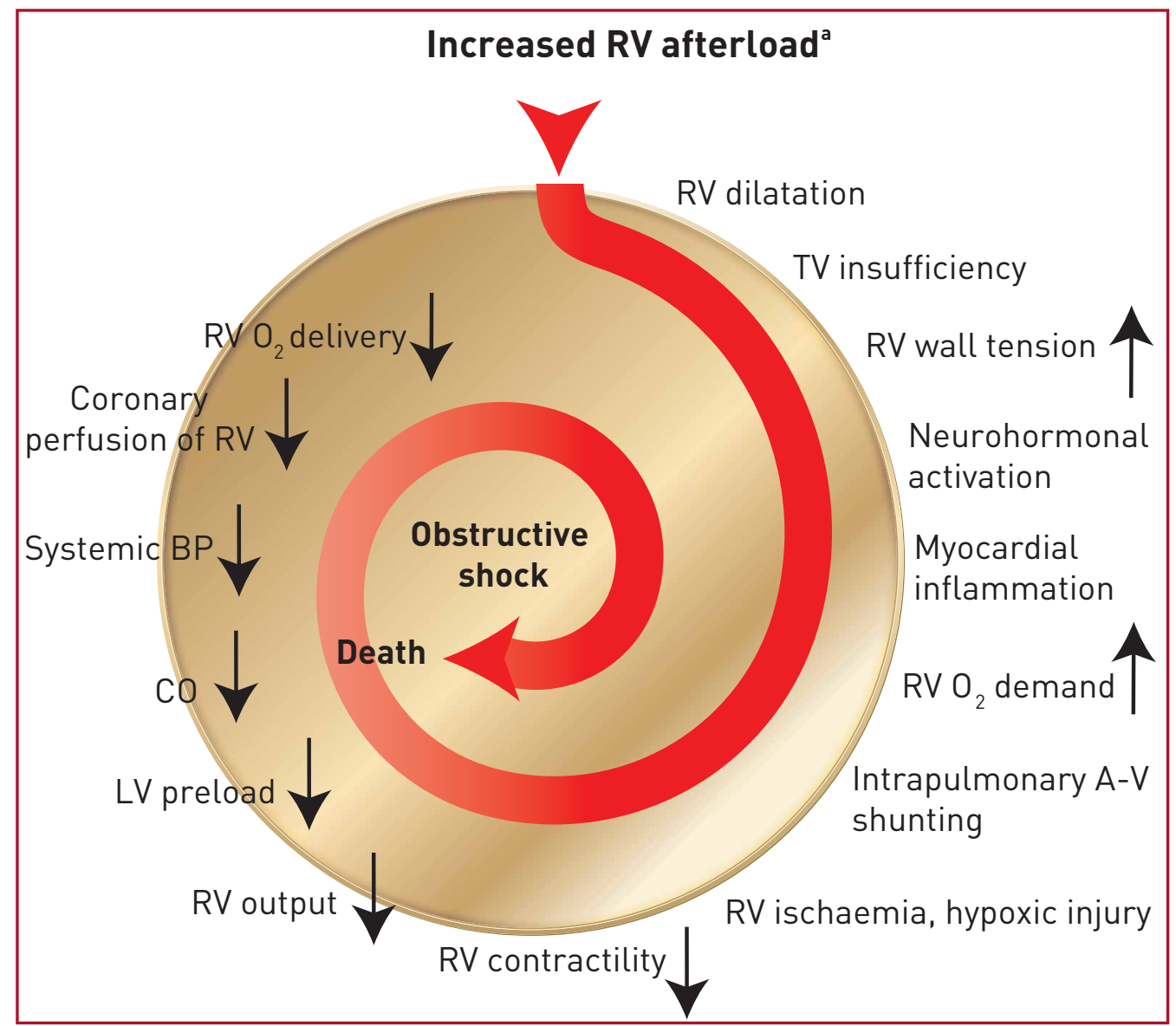

FIGURE 2 Key factors contributing to haemodynamic collapse and death in acute pulmonary embolism (modified from Konstantinides et al. [65] with permission). A-V:arterio-venous; BP: blood pressure; CO:

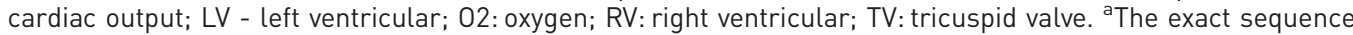
of events following the increase in RV afterload is not fully understood.

is a critical determinant of clinical severity and outcome in acute PE. Accordingly, clinical symptoms, and signs of overt RV failure and haemodynamic instability, indicate a high risk of early (in-hospital or 30 day) mortality. High-risk PE is defined by haemodynamic instability and encompasses the forms of clinical presentation shown in table 4.

As an immediately life-threatening situation, high-risk PE requires an emergency diagnostic (upon suspicion) and therapeutic (upon confirmation or if the level of suspicion is sufficiently high) strategy, as outlined in section 7. However, the absence of haemodynamic instability does not exclude beginning (and possibly progressing) RV dysfunction, and thus an elevated PE-related early risk. In this large population, further assessment (outlined in sections 5 and 7) is necessary to determine the level of risk and adjust management decisions accordingly.

\section{Diagnosis}

The increased awareness of venous thromboembolic disease and the ever-increasing availability of non-invasive imaging tests, mainly computed tomography (CT) pulmonary angiography (CTPA), have generated a tendency for clinicians to suspect and initiate a diagnostic workup for PE more frequently than in the past. This changing attitude is illustrated by the rates of PE confirmation among patients undergoing diagnostic workup: these were as low as $5 \%$ in recent North American diagnostic studies, in sharp contrast to the approximately $50 \%$ prevalence reported back in the early 1980s [71]. Therefore, it is critical that, when evaluating non-invasive diagnostic strategies for PE in the modern era, it is ensured that they are capable of safely excluding PE in contemporary patient populations with a rather low pre-test probability of the disease [72]. Conversely, a positive test should have an adequate specificity to set the indication for anticoagulant treatment.

\subsection{Clinical presentation}

The clinical signs and symptoms of acute PE are non-specific. In most cases, PE is suspected in a patient with dyspnoea, chest pain, pre-syncope or syncope, or haemoptysis [73-75]. Haemodynamic instability is 
a rare but important form of clinical presentation, as it indicates central or extensive PE with severely reduced haemodynamic reserve. Syncope may occur, and is associated with a higher prevalence of haemodynamic instability and RV dysfunction [76]. Conversely, and according to the results of a recent study, acute PE may be a frequent finding in patients presenting with syncope (17\%), even in the presence of an alternative explanation [77].

In some cases, PE may be asymptomatic or discovered incidentally during diagnostic workup for another disease.

Dyspnoea may be acute and severe in central PE; in small peripheral PE, it is often mild and may be transient. In patients with pre-existing heart failure or pulmonary disease, worsening dyspnoea may be the only symptom indicative of PE. Chest pain is a frequent symptom of PE and is usually caused by pleural irritation due to distal emboli causing pulmonary infarction [78]. In central PE, chest pain may have a typical angina character, possibly reflecting RV ischaemia, and requiring differential diagnosis from an acute coronary syndrome or aortic dissection.

In addition to symptoms, knowledge of the predisposing factors for VTE is important in determining the clinical probability of the disease, which increases with the number of predisposing factors present; however, in $40 \%$ of patients with $\mathrm{PE}$, no predisposing factors are found [79]. Hypoxaemia is frequent, but $\leqslant 40 \%$ of patients have normal arterial oxygen saturation $\left(S_{\mathrm{aO}_{2}}\right)$ and $20 \%$ have a normal alveolar-arterial oxygen gradient $[80,81]$. Hypocapnia is also often present. A chest X-ray is frequently abnormal and, although its findings are usually non-specific in PE, it may be useful for excluding other causes of dyspnoea or chest pain [82]. Electrocardiographic changes indicative of RV strain-such as inversion of $\mathrm{T}$ waves in leads V1-V4, a QR pattern in V1, a S1Q3T3 pattern, and incomplete or complete right bundle branch block-are usually found in more severe cases of PE [83]; in milder cases, the only abnormality may be sinus tachycardia, present in $40 \%$ of patients. Finally, atrial arrhythmias, most frequently atrial fibrillation, may be associated with acute PE.

\subsection{Assessment of clinical (pre-test) probability}

The combination of symptoms and clinical findings with the presence of predisposing factors for VTE allows the classification of patients with suspected PE into distinct categories of clinical or pre-test probability, which correspond to an increasing actual prevalence of confirmed PE. This pre-test assessment can be done either by implicit (empirical) clinical judgement or by using prediction rules. As the post-test (i.e. after an imaging test) probability of PE depends not only on the characteristics of the diagnostic test itself but also on the pre-test probability, this is a key step in all diagnostic algorithms for PE.

The value of empirical clinical judgement has been confirmed in several large series [84, 85]. Clinical judgement usually includes commonplace tests such as chest X-rays and electrocardiograms for differential diagnosis. However, as clinical judgement lacks standardization, several explicit clinical prediction rules have been developed. Of these, the most frequently used prediction rules are the revised Geneva rule (table 5) and the Wells rule (supplementary table 1) [86]. Both prediction rules have been simplified in an attempt to increase their adoption into clinical practice [87, 88]; the simplified versions have been externally validated $[89,90]$.

Regardless of the score used, the proportion of patients with confirmed PE can be expected to be $\sim 10 \%$ in the low-probability category, $30 \%$ in the moderate-probability category, and $65 \%$ in the high-probability category [92]. When the two-level classification is used, the proportion of patients with confirmed PE is $\sim 12 \%$ in the PE-unlikely category and $30 \%$ in the PE-likely category [92]. A direct prospective comparison of these rules confirmed a similar diagnostic performance [89].

TABLE 4 Definition of haemodynamic instability, which delineates acute high-risk pulmonary embolism lone of the following clinical manifestations at presentation)

\section{$\begin{array}{ll}\text { (1) Cardiac arrest } & \text { (2) Obstructive shock [68-70] }\end{array}$}

Need for cardiopulmonary resuscitation
Systolic BP $<90 \mathrm{mmHg}$ or vasopressors required to achieve a $B P \geqslant 90 \mathrm{mmHg}$ despite adequate filling status

And

End-organ hypoperfusion laltered mental status; cold, clammy skin; oliguria/anuria; increased serum lactate)

\section{(3) Persistent hypotension}

Systolic BP $<90 \mathrm{mmHg}$ or systolic BP drop $\geqslant 40 \mathrm{mmHg}$, lasting longer than $15 \mathrm{~min}$ and not caused by new-onset arrhythmia, hypovolaemia, or sepsis

BP: blood pressure. 
TABLE 5 The revised Geneva clinical prediction rule for pulmonary embolism

Items

Clinical decision rule points

Original version [91] Simplified version [87]

\section{Previous PE or DVT \\ Heart rate \\ $75-94$ b.p.m. \\ $\geqslant 95$ b.p.m.}

Surgery or fracture within the past month

Haemoptysis

Active cancer

Unilateral lower-limb pain

Pain on lower-limb deep venous palpation and

unilateral oedema

Age $>65$ years

Clinical probability

Three-level score

Low

Intermediate

High

Two-level score

PE-unlikely

PE-likely
3

3

3

5

2

2

2

3

4

$0-3$

4-10

$\geqslant 11$

$0-5$

$\geqslant 6$

1

1

(1)

b.p.m.: beats per minute; DVT: deep vein thrombosis; PE: pulmonary embolism.

\subsection{Avoiding overuse of diagnostic tests for pulmonary embolism}

Searching for PE in every patient with dyspnoea or chest pain may lead to high costs and complications of unnecessary tests. The Pulmonary Embolism Rule-out Criteria (PERC) were developed for emergency department patients with the purpose of selecting, on clinical grounds, patients whose likelihood of having $\mathrm{PE}$ is so low that diagnostic workup should not even be initiated [93]. They comprise eight clinical variables significantly associated with an absence of $\mathrm{PE}$ : age $<50$ years; pulse $<100$ beats per minute; $S_{\mathrm{aO}_{2}}$ >94\%; no unilateral leg swelling; no haemoptysis; no recent trauma or surgery; no history of VTE; and no oral hormone use. The results of a prospective validation study [94], and those of a randomized non-inferiority management study [95], suggested safe exclusion of PE in patients with low clinical probability who, in addition, met all criteria of the PERC rule. However, the low overall prevalence of PE in these studies $[94,95]$ does not support the generalizability of the results.

\subsection{D-dimer testing}

D-dimer levels are elevated in plasma in the presence of acute thrombosis because of simultaneous activation of coagulation and fibrinolysis. The negative predictive value of D-dimer testing is high, and a normal D-dimer level renders acute PE or DVT unlikely. On the other hand, the positive predictive value of elevated D-dimer levels is low and D-dimer testing is not useful for confirmation of PE. D-dimer is also more frequently elevated in patients with cancer [96, 97], in hospitalized patients $[89,98]$, in severe infection or inflammatory disease, and during pregnancy $[99,100]$. Accordingly, the number of patients in whom D-dimer must be measured to exclude one PE (number needed to test) rises from 3 in the general population of an emergency department to $\geqslant 10$ in the specific situations listed above.

As a number of $\mathrm{D}$-dimer assays are available, clinicians should become aware of the diagnostic performance of the test used in their own hospital. The quantitative enzyme-linked immunosorbent assay (ELISA) or ELISA-derived assays have a diagnostic sensitivity of $\geqslant 95 \%$, and can be used to exclude PE in patients with either low or intermediate pre-test probability. In the emergency department, a negative ELISA D-dimer can, in combination with clinical probability, exclude the disease without further testing in $\sim 30 \%$ of patients with suspected PE [101-103]. Outcome studies have shown that the 3 month thrombo-embolic risk was $<1 \%$ in patients with low or intermediate clinical probability who were left untreated on the basis of a negative test result [104].

\subsubsection{Age-adjusted D-dimer cut-offs}

The specificity of D-dimer in suspected PE decreases steadily with age to $\sim 10 \%$ in patients $>80$ years of age [105]. The use of age-adjusted cut-offs may improve the performance of D-dimer testing in the elderly. A multinational prospective management study evaluated a previously validated age-adjusted cut-off (agex10 $\mu \mathrm{g} / \mathrm{L}$, 
for patients aged $>50$ years) in a cohort of 3346 patients [106]. Patients with a normal age-adjusted D-dimer value did not undergo CTPA; they were left untreated and followed for a 3 month period. Among the 766 patients who were $\geqslant 75$ years of age, 673 had a non-high clinical probability. Use of the age-adjusted (instead of the "standard" $500 \mu \mathrm{g} / \mathrm{L}$ ) D-dimer cut-off increased the number of patients in whom PE could be excluded from 6.4 to $30 \%$, without additional false-negative findings [106].

\subsubsection{D-dimer cut-offs adapted to clinical probability}

A prospective management trial used the "YEARS" clinical decision rule, which consists of three clinical items of the Wells score (supplementary table 1) - namely signs of DVT, haemoptysis, and PE more likely than an alternative diagnosis-plus D-dimer concentrations [107]. PE was considered to be excluded in patients without clinical items and D-dimer levels $<1000 \mathrm{ng} / \mathrm{mL}$, or in patients with one or more clinical items and D-dimer levels $<500 \mathrm{ng} / \mathrm{mL}$. All other patients underwent CTPA. Of the 2946 patients (85\%) in whom PE was ruled out at baseline and who were left untreated, 18 [0.61\%, 95\% confidence interval (CI) 0.36-0.96\%] were diagnosed with symptomatic VTE during the 3 month follow-up. CTPA was avoided in $48 \%$ of the included patients using this algorithm, compared to $34 \%$ if the Wells rule and a fixed D-dimer threshold of $500 \mathrm{ng} / \mathrm{mL}$ would have been applied [107].

\subsubsection{Point-of-care D-dimer assays}

In certain situations, notably in community or primary care medicine, "on-the-spot" D-dimer testing may have advantages over referring a patient to a central laboratory for D-dimer testing. This may particularly apply to remote areas where access to healthcare is limited [108, 109]. However, point-of-care assays have a lower sensitivity and negative predictive value compared with laboratory-based $\mathrm{D}$-dimer tests. In a systematic review and meta-analysis, sensitivity of point-of-care D-dimer assays was 88\% (95\% CI 83-92\%) whereas conventional laboratory-based D-dimer testing had a sensitivity of at least 95\% [110]. As a result, point-of-care D-dimer assays should only be used in patients with a low pre-test probability. In these situations, PE could be ruled out in $46 \%$ of patients with suspected PE without proceeding to imaging tests (with a failure rate of $1.5 \%$ ), as suggested by a prospective study in Dutch primary care [111].

\subsection{Computed tomographic pulmonary angiography}

Multidetector CTPA is the method of choice for imaging the pulmonary vasculature in patients with suspected PE. It allows adequate visualization of the pulmonary arteries down to the subsegmental level [112-114]. The Prospective Investigation On Pulmonary Embolism Diagnosis (PIOPED) II study observed a sensitivity of $83 \%$ and a specificity of $96 \%$ for (mainly four-detector) CTPA in PE diagnosis [115]. PIOPED II also highlighted the influence of pre-test clinical probability on the predictive value of multidetector CTPA. In patients with a low or intermediate clinical probability of PE, a negative CTPA had a high negative predictive value for PE (96 and 89\%, respectively), but its negative predictive value was only $60 \%$ if the pre-test probability was high. Conversely, the positive predictive value of a positive CTPA was high (92-96\%) in patients with an intermediate or high clinical probability, but much lower (58\%) in patients with a low pre-test likelihood of PE [115]. Therefore, clinicians should consider further testing in case of discordance between clinical judgement and the CTPA result.

Several studies have provided evidence in favour of CTPA as a stand-alone imaging test for excluding PE. Taken together, the available data suggest that a negative CTPA result is an adequate criterion for the exclusion of PE in patients with low or intermediate clinical probability of PE. On the other hand, it remains controversial whether patients with a negative CTPA and a high clinical probability should be further investigated.

Chronic thromboembolic pulmonary hypertension $(\mathrm{CTEPH})$ is a potentially fatal late sequela of PE, but pre-existing CTEPH should not be missed in patients investigated for suspected acute PE. Signs of pre-existing CTEPH on CTPA are listed in supplementary table 2; the diagnosis and management of $\mathrm{CTEPH}$ is discussed in section 10 .

The major strengths, weaknesses/limitations, and radiation issues related to the use of CTPA in the diagnosis of $\mathrm{PE}$ are summarized in table 6.

\subsection{Lung scintigraphy}

The planar ventilation/perfusion [V/Q (lung scintigraphy)] scan is an established diagnostic test for suspected PE. Perfusion scans are combined with ventilation studies, for which multiple tracers such as xenon-133 gas, krypton-81 gas, technetium-99m-labelled aerosols, or technetium-99m-labelled carbon microparticles (Technegas) can be used. The purpose of the ventilation scan is to increase specificity: in acute PE, ventilation is expected to be normal in hypoperfused segments (mismatched). Being a lower-radiation and contrast medium-sparing procedure, the V/Q scan may preferentially be applied in outpatients with a low clinical probability and a normal chest X-ray, in young (particularly female) patients, in pregnant women, in patients with history of contrast medium-induced anaphylaxis, and patients with severe renal failure [116]. 
TABLE 6 Imaging tests for diagnosis of pulmonary embolism

\begin{tabular}{|c|c|c|c|}
\hline & Strengths & Weaknesses/limitations & Radiation issues $^{a}$ \\
\hline CTPA & $\begin{array}{l}\text { - Readily available around the clock } \\
\text { in most centres } \\
\text { - Excellent accuracy } \\
\text { - Strong validation in prospective } \\
\text { management outcome studies } \\
\text { - Low rate of inconclusive results } \\
(3-5 \%) \\
\text { - May provide alternative diagnosis if } \\
\text { PE excluded } \\
\text { - Short acquisition time }\end{array}$ & $\begin{array}{l}\text { - Radiation exposure } \\
\text { - Exposure to iodine contrast: } \\
\text { - limited use in iodine allergy and } \\
\text { - } \text { ryperthyroidism in pregnant and breastfeeding } \\
\text { women } \\
\text { - contraindicated in severe renal } \\
\text { failure } \\
\text { - Tendency to overuse because of easy } \\
\text { accessibility } \\
\text { - Clinical relevance of CTPA diagnosis of } \\
\text { subsegmental PE unknown }\end{array}$ & $\begin{array}{l}\text { - Radiation effective dose } \\
3-10 \mathrm{mSv^{b }} \\
\text { - Significant radiation exposure to } \\
\text { young female breast tissue }\end{array}$ \\
\hline Planar V/Q scan & $\begin{array}{l}\text { - Almost no contraindications } \\
\text { - Relatively inexpensive } \\
\text { - Strong validation in prospective } \\
\text { management outcome studies }\end{array}$ & $\begin{array}{l}\text { - Not readily available in all centres } \\
\text { - Interobserver variability in interpretation } \\
\text { - Results reported as likelihood ratios } \\
\text { - Inconclusive in } 50 \% \text { of cases } \\
\text { - Cannot provide alternative diagnosis if } \\
\text { PE excluded }\end{array}$ & $\begin{array}{l}\text { - Lower radiation than CTPA, } \\
\text { effective dose } \sim 2 \mathrm{mSv}^{\mathrm{b}}\end{array}$ \\
\hline V/Q SPECT & $\begin{array}{l}\text { - Almost no contraindications } \\
\text { - Lowest rate of non-diagnostic tests } \\
\text { (<3\%) } \\
\text { - High accuracy according to } \\
\text { available data } \\
\text { - Binary interpretation ("PE" versus } \\
\text { "no PE") }\end{array}$ & $\begin{array}{l}\text { - Variability of techniques } \\
\text { - Variability of diagnostic criteria } \\
\text { - Cannot provide alternative diagnosis if } \\
\text { PE excluded } \\
\text { - No validation in prospective } \\
\text { management outcome studies }\end{array}$ & $\begin{array}{l}\text { - Lower radiation than CTPA, } \\
\text { effective dose } \sim 2 \mathrm{mSv}^{\mathrm{b}}\end{array}$ \\
\hline $\begin{array}{l}\text { Pulmonary } \\
\text { angiography }\end{array}$ & - Historical gold standard & $\begin{array}{l}\text { - Invasive procedure } \\
\text { - Not readily available in all centres }\end{array}$ & $\begin{array}{l}\text { - Highest radiation, effective dose } \\
10-20 \mathrm{mSv}^{\mathrm{b}}\end{array}$ \\
\hline \multicolumn{4}{|c|}{$\begin{array}{l}\text { CTPA: computed tomographic pulmonary angiography; } \mathrm{mGy} \text { : milligray; mSv: millisieverts; PE: pulmonary embolism; SPECT: single-photon } \\
\text { emission computed tomography; } \mathrm{V} / \mathrm{Q} \text { : ventilation/perfusion (lung scintigraphy). a In this section, effective radiation dose is expressed in mSV } \\
\text { [dose in } \mathrm{mSv}=\text { absorbed dose in } \mathrm{mGy \times radiation} \text { weighting factor (1.0 for } \mathrm{X} \text {-rays) } \times \text { tissue weighting factor]. This reflects the effective doses of all } \\
\text { organs that have been exposed, that is, the overall radiation dose to the body from the imaging test. Compare with table } 12 \text {, in which the } \\
\text { absorbed radiation dose is expressed in } \mathrm{mGy} \text { to reflect the radiation exposure to single organs or to the fetus. }{ }^{b} \text { For comparison, the } \\
\text { whole-body effective dose of a chest } \mathrm{X} \text {-ray examination is } 0.1 \mathrm{mSv}[141] \text {. }\end{array}$} \\
\hline
\end{tabular}

Planar lung scan results are frequently classified according to the criteria established in the PIOPED study [117]. These criteria were the subject of debate and have been revised [118, 119]. To facilitate communication with clinicians, a three-tier classification is preferable: normal scan (excluding PE), high-probability scan (considered diagnostic of PE in most patients), and non-diagnostic scan [120-122]. Prospective clinical outcome studies suggested that it is safe to withhold anticoagulant therapy in patients with a normal perfusion scan. This was confirmed by a randomized trial comparing the V/Q scan with CTPA [122]. An analysis from the PIOPED II study suggested that a high-probability V/Q scan could confirm $\mathrm{PE}$, although other sources suggest that the positive predictive value of a high-probability lung scan is not sufficient to confirm PE in patients with a low clinical probability $[123,124]$.

Performing only a perfusion scan might be acceptable in patients with a normal chest X-ray; any perfusion defect in this situation would be considered a mismatch. The high frequency of nondiagnostic scans is a limitation because they indicate the necessity for further diagnostic testing. Various strategies to overcome this problem have been proposed, notably the incorporation of clinical probability. Although the use of perfusion scanning and chest X-ray with the Prospective Investigative Study of Acute Pulmonary Embolism Diagnosis (PISAPED) criteria may be associated with a low rate of inconclusive results, the sensitivity appears too low to exclude PE and thus this approach may be less safe than CTPA [123, 125].

Several studies suggest that data acquisition in single-photon emission CT (SPECT) imaging, with or without low-dose CT, may decrease the proportion of non-diagnostic scans to as low as $0-5 \%$ [121, 126-128]. However, most studies reporting on the accuracy of SPECT are limited by their retrospective design $[129,130]$ or the inclusion of SPECT itself in the reference standard [127], and only one study used a validated diagnostic algorithm [131]. The diagnostic criteria for SPECT also varied; most studies defined PE as one 
or two subsegmental perfusion defects without ventilation defects, but these criteria are infrequently used in clinical practice. In addition, the optimal scanning technique (perfusion SPECT, V/Q SPECT, perfusion SPECT with non-enhanced CT, or V/Q SPECT with non-enhanced CT) remains to be defined. Finally, few outcome studies are available, and with incomplete follow-up [132]. Large-scale prospective studies are needed to validate SPECT techniques.

The major strengths, weaknesses/limitations, and radiation issues related to the use of V/Q scan and V/Q SPECT in the diagnosis of PE are summarized in table 6.

\subsection{Pulmonary angiography}

For several decades, pulmonary angiography was the "gold standard" for the diagnosis or exclusion of acute PE, but it is now rarely performed as less-invasive CTPA offers similar diagnostic accuracy [133]. The diagnosis of acute PE is based on direct evidence of a thrombus in two projections, either as a filling defect or as amputation of a pulmonary arterial branch [134]. Thrombi as small as 1-2 mm within the subsegmental arteries can be visualized by digital subtraction angiography, but there is substantial interobserver variability at this level $[135,136]$.

Pulmonary angiography is not free of risk. In a study of 1111 patients, procedure-related mortality was $0.5 \%$, major non-fatal complications occurred in $1 \%$, and minor complications in 5\% [137]. The majority of deaths occurred in patients with haemodynamic compromise or respiratory failure. The amount of contrast agent should be reduced and non-selective injections avoided in patients with haemodynamic compromise [138].

The major strengths, weaknesses/limitations, and radiation issues related to the use of pulmonary angiography in the diagnosis of PE are summarized in table 6.

\subsection{Magnetic resonance angiography}

Magnetic resonance angiography (MRA) has been evaluated for several years regarding suspected PE. However, the results of large-scale studies $[139,140]$ show that this technique, although promising, is not yet ready for clinical practice due to its low sensitivity, the high proportion of inconclusive MRA scans, and its low availability in most emergency settings. The hypothesis that a negative MRA, combined with the absence of proximal DVT on compression ultrasonography (CUS), may safely rule out clinically significant $\mathrm{PE}$ is currently being investigated in an ongoing multicentre outcome study [Clinicaltrials.gov National Clinical Trial (NCT) number 02059551].

\subsection{Echocardiography}

Acute PE may lead to RV pressure overload and dysfunction, which can be detected by echocardiography. Given the peculiar geometry of the RV, there is no individual echocardiographic parameter that provides fast and reliable information on RV size or function. This is why echocardiographic criteria for the diagnosis of PE have differed between studies. Because of the reported negative predictive value of 40-50\%, a negative result cannot exclude PE $[124,142,143]$. On the other hand, signs of RV overload or dysfunction may also be found in the absence of acute PE, and may be due to concomitant cardiac or respiratory disease [144].

Echocardiographic findings of RV overload and/or dysfunction are graphically presented in Figure 3. RV dilation is found in $\geqslant 25 \%$ of patients with PE on transthoracic echocardiography (TTE) and is useful for risk stratification of the disease [145]. More specific echocardiographic findings were reported to retain a high positive predictive value for PE even in the presence of pre-existing cardiorespiratory disease. Thus, the combination of a pulmonary ejection acceleration time (measured in the RV outflow tract) $<60 \mathrm{~ms}$ with a peak systolic tricuspid valve gradient $<60 \mathrm{mmHg}$ ("60/60" sign), or with depressed contractility of the RV free wall compared to the "echocardiographic" RV apex (McConnell sign), is suggestive of PE [146]. However, these findings are present in only $\sim 12$ and $20 \%$ of unselected PE patients, respectively [145]. Detection of echocardiographic signs of RV pressure overload helps to distinguish acute PE from RV free wall hypokinesia or akinesia due to RV infarction, which may mimic the McConnell sign [147]. It should be noted that in $\sim 10 \%$ of PE patients, echocardiography can show potentially misleading incidental findings such as significant LV systolic dysfunction or valvular heart disease [145]. Decreased tricuspid annular plane systolic excursion (TAPSE) may also be present in PE patients [148, 149]. Echocardiographic parameters of RV function derived from Doppler tissue imaging and wall strain assessment may also be affected by the presence of acute PE (figure 3). However, they probably have low sensitivity as stand-alone findings, as they were reported to be normal in haemodynamically stable patients despite the presence of PE $[150,151]$.

Echocardiographic examination is not mandatory as part of the routine diagnostic workup in haemodynamically stable patients with suspected PE [124], although it may be useful in the differential 


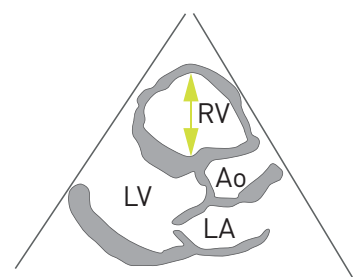

a) Enlarged right ventricle, parasternal long axis view

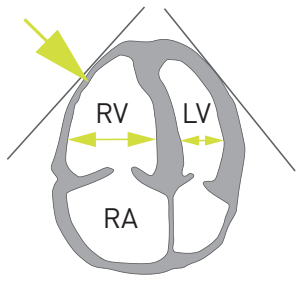

b) Dilated RV with basal RV/LV ratio $>1.0$, and McConnell sign (arrow), four chamber view

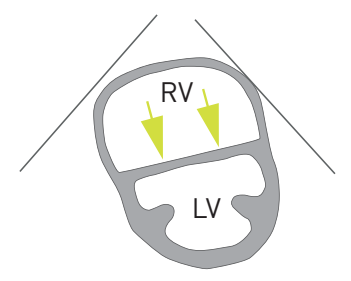

c) Flattened intraventricle septum (arrows) parasternal short axis view

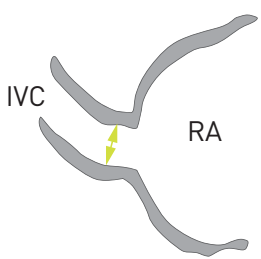

d) Distended inferior vena cava with diminished inspiratory collapsibility, subcostal view

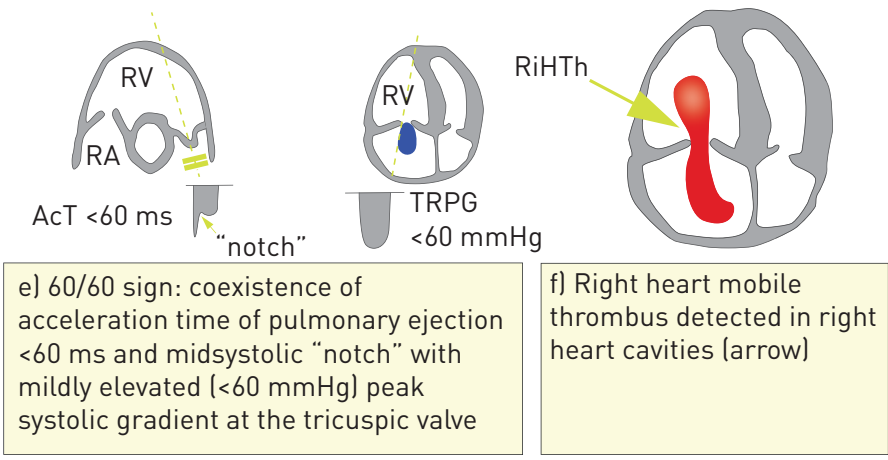

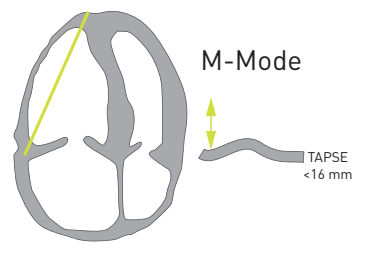

g) Decreased tricuspid annular plane systolic excursion (TAPSE) measured with M-Mode $(<16 \mathrm{~mm})$

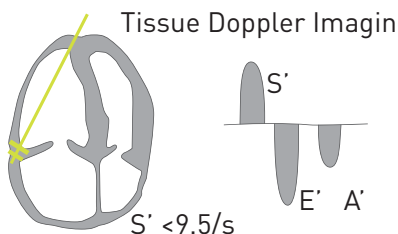

h) Decreased peak systolic (S') velocity of tricuspid annulus $(<9.5 \mathrm{~cm} / \mathrm{s})$

FIGURE 3 Graphic representation of transthoracic echocardiographic parameters in the assessment of right ventricular pressure overload. A': peak late diastolic (during atrial contraction) velocity of tricuspid annulus by tissue Doppler imaging; AcT: right ventricular outflow Doppler acceleration time; Ao: aorta; $E^{\prime}$ : peak early diastolic velocity of tricuspid annulus by tissue Doppler imaging; IVC: inferior vena cava; LA: left atrium; LV: left ventricle; RA: right atrium; RiHTh: right heart thrombus (or thrombi); RV: right ventricle/ventricular; $\mathrm{S}^{\prime}$ : peak systolic velocity of tricuspid annulus by tissue Doppler imaging; TAPSE: tricuspid annular plane systolic excursion; TRPG: tricuspid valve peak systolic gradient.

diagnosis of acute dyspnoea. This is in contrast to suspected high-risk PE, in which the absence of echocardiographic signs of RV overload or dysfunction practically excludes PE as the cause of haemodynamic instability. In the latter case, echocardiography may be of further help in the differential diagnosis of the cause of shock, by detecting pericardial tamponade, acute valvular dysfunction, severe global or regional LV dysfunction, aortic dissection, or hypovolaemia [152]. Conversely, in a haemodynamically compromised patient with suspected $\mathrm{PE}$, unequivocal signs of $\mathrm{RV}$ pressure overload, especially with more specific echocardiographic findings (60/60 sign, McConnell sign, or right-heart thrombi), justify emergency reperfusion treatment for PE if immediate CT angiography is not feasible in a patient with high clinical probability and no other obvious causes for RV pressure overload [152].

Mobile right-heart thrombi are detected by TTE or transoesophageal echocardiography (TOE), or by CT angiography, in $<4 \%$ of unselected patients with PE [153-155]. Their prevalence may reach $18 \%$ among $\mathrm{PE}$ patients in the intensive care setting [156]. Mobile right-heart thrombi essentially confirm the diagnosis of $\mathrm{PE}$ and are associated with high early mortality, especially in patients with RV dysfunction [155, 157-159].

In some patients with suspected acute PE, echocardiography may detect increased RV wall thickness or tricuspid insufficiency jet velocity beyond values compatible with acute RV pressure overload ( $>3.8 \mathrm{~m} / \mathrm{s}$ or a tricuspid valve peak systolic gradient $>60 \mathrm{mmHg}$ ) [160]. In these cases, chronic thromboembolic (or other) pulmonary hypertension ( $\mathrm{PH}$ ) should be included in the differential diagnosis.

\subsection{Compression ultrasonography}

In the majority of cases, PE originates from DVT in a lower limb, and only rarely from upper-limb DVT (mostly following venous catheterization). In a study using venography, DVT was found in $70 \%$ of patients with proven PE [161]. Nowadays, lower-limb CUS has largely replaced venography for diagnosing DVT. CUS has a sensitivity $>90 \%$ and a specificity of $\sim 95 \%$ for proximal symptomatic DVT [162, 163]. CUS shows a DVT in 30-50\% of patients with PE [162-164], and finding a proximal DVT in patients suspected of having PE is considered sufficient to warrant anticoagulant treatment without further testing [165]. However, patients in whom PE is indirectly confirmed by the presence of a proximal DVT should undergo risk assessment for PE severity and the risk of early death. 
In the setting of suspected PE, CUS can be limited to a simple four-point examination (bilateral groin and popliteal fossa). The only validated diagnostic criterion for DVT is incomplete compressibility of the vein, which indicates the presence of a clot, whereas flow measurements are unreliable. A positive proximal CUS result has a high positive predictive value for PE. The high diagnostic specificity (96\%) along with a low sensitivity (41\%) of CUS in this setting was shown by a recent meta-analysis $[165,166]$. CUS is a useful procedure in the diagnostic strategy of patients with $\mathrm{CT}$ contraindications. The probability of a positive proximal CUS in suspected PE is higher in patients with signs and symptoms related to the leg veins than in asymptomatic patients $[162,163]$.

In patients admitted to the emergency department with haemodynamic instability and suspicion of PE, a combination of venous ultrasound with cardiac ultrasound may further increase specificity. Conversely, an echocardiogram without signs of RV dysfunction and a normal venous ultrasound excluded PE with a high (96\%) negative predictive value in one study [167].

For further details on the diagnosis and management of DVT, the reader is referred to the joint consensus document of the ESC Working Groups of Aorta and Peripheral Vascular Diseases, and Pulmonary Circulation and Right Ventricular Function [1].

\subsection{Recommendations for diagnosis}

\section{Recommendations}

Suspected PE with haemodynamic instability

In suspected high-risk PE, as indicated by the presence of haemodynamic instability, bedside echocardiography or emergency CTPA (depending on availability and clinical circumstances) is recommended for diagnosis [169].

It is recommended that i.v. anticoagulation with UFH, including a weight-adjusted bolus injection, be initiated without delay in patients with suspected high-risk PE.

\section{Suspected PE without haemodynamic instability}

The use of validated criteria for diagnosing PE is recommended [12].

Initiation of anticoagulation is recommended without delay in patients with high or intermediate clinical probability of PE while diagnostic workup is in progress.

\section{Clinical evaluation}

It is recommended that the diagnostic strategy be based on clinical probability, assessed either by clinical judgement or by a validated prediction rule $[89,91,92,103,134,170-172]$.

\section{D-dimer}

Plasma D-dimer measurement, preferably using a highly sensitive assay, is recommended in outpatients/emergency department patients with low or intermediate clinical probability, or those that are PE-unlikely, to reduce the need for unnecessary imaging and irradition $[101-103,122,164,171,173,174]$.

As an alternative to the fixed D-dimer cut-off, a negative D-dimer test using an age-adjusted cut-off (age $\times 10 \mu \mathrm{g} / \mathrm{L}$, in patients aged $>50$ years) should be considered for excluding PE in patients with low or intermediate clinical probability, or those that are PE-unlikely [106].

As an alternative to the fixed or age-adjusted D-dimer cut-off, D-dimer levels adapted to clinical probabilityc should be considered to exclude PE [107].

D-dimer measurement is not recommended in patients with high clinical probability, as a normal result does not safely exclude PE, even when using a highly sensitive assay [175, 176].

\section{CTPA}

It is recommended to reject the diagnosis of PE (without further testing) if CTPA is normal in a patient with low or intermediate clinical probability, or who is PE-unlikely $[101,122,164$, 171].

It is recommended to accept the diagnosis of PE (without further testing) if CTPA shows a segmental or more proximal filling defect in a patient with intermediate or high clinical probability [115].

It should be considered to reject the diagnosis of PE (without further testing) if CTPA is normal in a patient with high clinical probability or who is PE-likely [171].

Further imaging tests to confirm PE may be considered in cases of isolated subsegmental filling defects [115].

CT venography is not recommended as an adjunct to CTPA $[115,164]$.

\section{Classa Levelb}
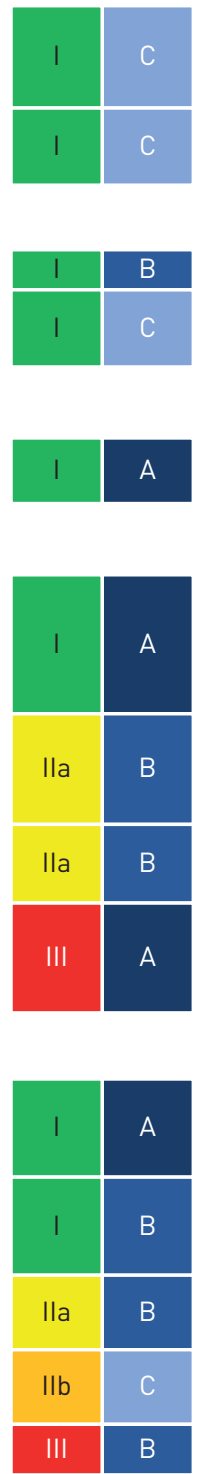


\section{V/Q scintigraphy}

It is recommended to reject the diagnosis of PE (without further testing) if the perfusion lung scan is normal $[75,122,134,174]$.

It should be considered to accept that the diagnosis of $P E$ (without further testing) if the $V / Q$ scan yields high probability for PE [134].

A non-diagnostic $V / Q$ scan should be considered as exclusion of $P E$ when combined with a negative proximal CUS in patients with low clinical probability, or who are PE-unlikely [75, $122,174]$.

\section{V/Q SPECT}

V/Q SPECT may be considered for PE diagnosis [121, 126-128].

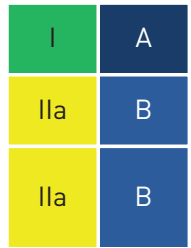

\section{Lower-limb CUS}

It is recommended to accept the diagnosis of VTE (and PE) if a CUS shows a proximal DVT in a patient with clinical suspicion of PE $[164,165]$.

If CUS shows only a distal DVT, further testing should be considered to confirm PE [177].

If a positive proximal CUS is used to confirm PE, assessment of PE severity should be considered to permit risk-adjusted management $[178,179]$.

\section{MRA}

MRA is not recommended for ruling out PE $[139,140]$

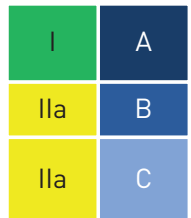

CT: computed tomographic; CTPA: computed tomography pulmonary angiography/angiogram; CUS: compression ultrasonography; DVT: deep vein thrombosis; i.v.: intravenous; MRA: magnetic resonance angiography; PE: pulmonary embolism; SPECT: single-photon emission computed tomography; UFH: unfractionated heparin; V/Q: ventilation/perfusion (lung scintigraphy); VTE: venous thromboembolism. ${ }^{a}$ Class of recommendation. ${ }^{b}$ Level of evidence. ${ }^{C} D$-dimer cut-off levels adapted to clinical probability according to the YEARS model (signs of DVT, haemoptysis, and whether an alternative diagnosis is less likely than PE) may be used. According to this model, PE is excluded in patients without clinical items and D-dimer levels $<1000 \mu \mathrm{g} / \mathrm{L}$, or in patients with one or more clinical items and D-dimer levels $<500 \mu \mathrm{g} / \mathrm{L}$ [107]. ' Low level of recommendation in view of the limitations summarized in table 5.

\subsection{Computed tomography venography}

When using CTPA, it is possible to image the deep veins of the legs during the same acquisition [115]. However, this approach has not been widely validated and the added value of venous imaging is limited [164]. Moreover, using CT venography is associated with increased radiation doses [168].

\section{Assessment of pulmonary embolism severity and the risk of early death}

Risk stratification of patients with acute PE is mandatory for determining the appropriate therapeutic management approach. As described in section 3.3, initial risk stratification is based on clinical symptoms and signs of haemodynamic instability (table 4), which indicate a high risk of early death. In the large remaining group of patients with PE who present without haemodynamic instability, further (advanced) risk stratification requires the assessment of two sets of prognostic criteria: (i) clinical, imaging, and laboratory indicators of PE severity, mostly related to the presence of RV dysfunction; and (ii) presence of comorbidity and any other aggravating conditions that may adversely affect early prognosis.

\subsection{Clinical parameters of pulmonary embolism severity}

Acute RV failure, defined as a rapidly progressive syndrome with systemic congestion resulting from impaired RV filling and/or reduced RV flow output [68], is a critical determinant of outcome in acute PE. Tachycardia, low systolic BP, respiratory insufficiency (tachypnoea and/or low $S_{\mathrm{aO}_{2}}$ ), and syncope, alone or in combination, have been associated with an unfavourable short-term prognosis in acute PE.

\subsection{Imaging of right ventricular size and function}

\subsubsection{Echocardiography}

Echocardiographic parameters used to stratify the early risk of patients with PE are graphically presented in figure 3, and their prognostic values are summarized in supplementary table 3. Of these, an RV/LV diameter ratio $\geqslant 1.0$ and a TAPSE $<16 \mathrm{~mm}$ are the findings for which an association with unfavourable prognosis has most frequently been reported [148].

Overall, evidence for RV dysfunction on echocardiography is found in $\geqslant 25 \%$ of unselected patients with acute PE [145]. Systematic reviews and meta-analyses have suggested that RV dysfunction on echocardiography is associated with an elevated risk of short-term mortality in patients who appear 
haemodynamically stable at presentation $[180,181]$, but its overall positive predictive value for PE-related death was low $(<10 \%)$ in a meta-analysis [180]. This weakness is partly related to the fact that echocardiographic parameters have proved difficult to standardize $[148,180]$. Nevertheless, echocardiographic assessment of the morphology and function of the RV is widely recognized as a valuable tool for the prognostic assessment of normotensive patients with acute PE in clinical practice.

In addition to RV dysfunction, echocardiography can identify right-to-left shunt through a patent foramen ovale and the presence of right heart thrombi, both of which are associated with increased mortality in patients with acute PE $[67,158]$. A patent foramen ovale also increases the risk of ischaemic stroke due to paradoxical embolism in patients with acute PE and RV dysfunction [182, 183].

\subsubsection{Computed tomographic pulmonary angiography}

CTPA parameters used to stratify the early risk of patients with PE are summarized in supplementary table 3. Four-chamber views of the heart by CT angiography can detect RV enlargement (RV end-diastolic diameter and RV/LV ratio measured in the transverse or four-chamber view) as an indicator of RV dysfunction. The prognostic value of an enlarged RV is supported by the results of a prospective multicentre cohort study in 457 patients [184]. In that study, RV enlargement (defined as an RV/LV ratio $\geqslant 0.9$ ) was an independent predictor of an adverse in-hospital outcome, both in the overall population with PE [hazard ratio (HR) 3.5, 95\% CI 1.6-7.7] and in haemodynamically stable patients (HR 3.8, 95\% CI 1.3-10.9) [184]. A meta-analysis of 49 studies investigating $>13000$ patients with PE confirmed that an increased RV/LV ratio of $\geqslant 1.0$ on CT was associated with a 2.5 -fold increased risk for all-cause mortality [odds ratio (OR) $2.5,95 \%$ CI 1.8-3.5], and with a five-fold risk for PE-related mortality (OR 5.0, 95\% CI 2.7-9.2) [185].

Mild RV dilation (RV/LV slightly above 0.9 ) on CT is a frequent finding ( $>50 \%$ of haemodynamically stable PE patients [186]), but it probably has minor prognostic significance. However, increasing RV/LV diameter ratios are associated with rising prognostic specificity $[187,188]$, even in patients considered to be at "low" risk on the basis of clinical criteria [186]. Thus, RV/LV ratios $\geqslant 1.0$ (instead of 0.9) on CT angiography may be more appropriate to indicate poor prognosis.

Apart from RV size and the RV/LV ratio, CT may provide further prognostic information based on volumetric analysis of the heart chambers [189-191] and assessment of contrast reflux to the inferior vena cava (IVC) $[185,192,193]$.

\subsection{Laboratory biomarkers}

5.3.1. Markers of myocardial injury

Elevated plasma troponin concentrations on admission may be associated with a worse prognosis in the acute phase of PE. Cardiac troponin I or $\mathrm{T}$ elevation are defined as concentrations above the normal limits, and thresholds depend on the assay used; an overview of the cut-off values has been provided by a meta-analysis [194]. Of patients with acute PE, between 30 (using conventional assays) [194, 195] and 60\% (using high-sensitivity assays) [196, 197] have elevated cardiac troponin I or T concentrations. A meta-analysis showed that elevated troponin concentrations were associated with an increased risk of mortality, both in unselected patients (OR 5.2, 95\% CI 3.3-8.4) and in those who were haemodynamically stable at presentation (OR 5.9, 95\% CI 2.7-13.0) [195].

On their own, increased circulating levels of cardiac troponins have relatively low specificity and positive predictive value for early mortality in normotensive patients with acute PE. However, when interpreted in combination with clinical and imaging findings, they may improve the identification of an elevated PE-related risk and the further prognostic stratification of such patients (supplementary table 4). At the other end of the severity spectrum, high-sensitivity troponin assays possess a high negative predictive value in the setting of acute PE [197]. For example, in a prospective multicentre cohort of 526 normotensive patients, high-sensitivity troponin $\mathrm{T}$ concentrations $<14 \mathrm{pg} / \mathrm{mL}$ had a negative predictive value of $98 \%$ for excluding an adverse in-hospital clinical outcome [63]. Age-adjusted high-sensitivity troponin $\mathrm{T}$ cut-off values $(\geqslant 14 \mathrm{pg} / \mathrm{mL}$ for patients aged $<75$ years and $\geqslant 45 \mathrm{pg} / \mathrm{mL}$ for those $\geqslant 75$ years) may further improve the negative predictive value of this biomarker [196].

Heart-type fatty acid-binding protein (H-FABP), an early and sensitive marker of myocardial injury, provides prognostic information in acute PE, both in unselected [198, 199] and normotensive patients $[200,201]$. In a meta-analysis investigating 1680 patients with PE, H-FABP concentrations $\geqslant 6 \mathrm{ng} / \mathrm{mL}$ were associated with an adverse short-term outcome (OR 17.7, 95\% CI 6.0-51.9) and all-cause mortality (OR 32.9, 95\% CI 8.8-123.2) [202].

\subsubsection{Markers of right ventricular dysfunction}

$\mathrm{RV}$ pressure overload due to acute PE is associated with increased myocardial stretch, which leads to the release of B-type natriuretic peptide (BNP) and N-terminal (NT)-proBNP. Thus, the plasma levels of 
natriuretic peptides reflect the severity of RV dysfunction and haemodynamic compromise in acute $\mathrm{PE}$ [203]. A meta-analysis found that $51 \%$ of 1132 unselected patients with acute PE had elevated BNP or NT-proBNP concentrations on admission; these patients had a $10 \%$ risk of early death (95\% CI 8.0-13\%) and a 23\% (95\% CI 20-26\%) risk of an adverse clinical outcome [204].

Similar to cardiac troponins (see above), elevated BNP or NT-proBNP concentrations possess low specificity and positive predictive value (for early mortality) in normotensive patients with PE [205], but low levels of BNP or NT-proBNP are capable of excluding an unfavourable early clinical outcome, with high sensitivity and a negative predictive value [180]. In this regard, an NT-proBNP cut-off value $<500 \mathrm{pg} / \mathrm{mL}$ was used to select patients for home treatment in a multicentre management study [206]. If emphasis is placed on increasing the prognostic specificity for an adverse early outcome, higher cut-off values $\geqslant 600 \mathrm{pg} / \mathrm{mL}$ might be more appropriate [207].

\subsubsection{Other laboratory biomarkers}

Lactate is a marker of imbalance between tissue oxygen supply and demand, and consequently of severe $\mathrm{PE}$ with overt or imminent haemodynamic compromise. Elevated arterial plasma levels $\geqslant 2 \mathrm{mmol} / \mathrm{L}$ predict PE-related complications, both in unselected [208] and in initially normotensive [209, 210] PE patients.

Elevated serum creatinine levels and a decreased (calculated) glomerular filtration rate are related to 30 day all-cause mortality in acute PE [211]. Elevated neutrophil gelatinase-associated lipocalin and cystatin C, both indicating acute kidney injury, are also of prognostic value [212].

A recent meta-analysis investigating 18616 patients with acute PE found that hyponatraemia predicted in-hospital mortality (OR 5.6, 95\% CI 3.4-9.1) [213].

Vasopressin is released upon endogenous stress, hypotension, and low CO. Its surrogate marker, copeptin, has been reported to be useful for risk stratification of patients with acute PE [214, 215]. In a single-centre derivation study investigating 268 normotensive PE patients, copeptin levels $\geqslant 24 \mathrm{pmol} / \mathrm{L}$ were associated with a 5.4-fold (95\% CI 1.7-17.6) increased risk of an adverse outcome [216]. These results were confirmed in 843 normotensive PE patients prospectively included in three European cohorts [217].

\subsection{Combined parameters and scores for assessment of pulmonary embolism severity}

In patients who present without haemodynamic instability, individual baseline findings may not suffice to determine and further classify PE severity and PE-related early risk when used as stand-alone parameters. As a result, various combinations of the clinical, imaging, and laboratory parameters described above have been used to build prognostic scores, which permit a (semi)quantitative assessment of early PE-related risk of death. Of these, the Bova [218-221] and the H-FABP (or high-sensitivity troponin T), Syncope, Tachycardia (FAST) scores $[219,222,223]$ have been validated in cohort studies (supplementary table 4). However, their implications for patient management remain unclear. To date, only a combination of RV dysfunction on an echocardiogram (or CTPA) with a positive cardiac troponin test has directly been tested as a guide for early therapeutic decisions (anticoagulation plus reperfusion treatment versus anticoagulation alone) in a large randomized controlled trial (RCT) of PE patients presenting without haemodynamic instability [224].

\subsection{Integration of aggravating conditions and comorbidity into risk assessment of acute pulmonary embolism}

In addition to the clinical, imaging, and laboratory findings, which are directly linked to PE severity and PE-related early death, baseline parameters related to aggravating conditions and comorbidity are necessary to assess a patient's overall mortality risk and early outcome. Of the clinical scores integrating PE severity and comorbidity, the Pulmonary Embolism Severity Index (PESI) (table 7) is the one that has been most extensively validated to date [225-228]. The principal strength of the PESI lies in the reliable identification of patients at low risk for 30 day mortality (PESI classes I and II). One randomized trial employed a low PESI as the principal inclusion criterion for home treatment of acute PE [178].

In view of the complexity of the original PESI, which includes 11 differently weighed variables, a simplified version (sPESI; table 7) has been developed and validated [229-231]. As with the original version of the PESI, the strength of the sPESI lies in the reliable identification of patients at low risk for 30 day mortality. The prognostic performance of the sPESI has been confirmed in observational cohort studies [227, 228], although this index has not yet been prospectively used to guide therapeutic management of low-risk PE patients.

The diagnosis of concomitant DVT has been identified as an adverse prognostic factor, being independently associated with death within the first 3 months after acute PE [232]. In a meta-analysis investigating 8859 patients with $\mathrm{PE}$, the presence of concomitant DVT was confirmed as a predictor of 30 day all-cause mortality (OR 1.9, 95\% CI 1.5-2.4), although it did not predict PE-related adverse outcomes at 90 days [233]. Thus, concomitant DVT can be regarded as an indicator of significant comorbidity in acute PE. 
TABLE 7 Original and simplified Pulmonary Embolism Severity Index

Parameter Original version [226]

Simplified version [229]

\section{Age}

Male sex

Cancer

Chronic heart failure

Chronic pulmonary disease

Pulse rate $\geqslant 110$ b.p.m.

Systolic BP $<100 \mathrm{mmHg}$

Respiratory rate $>30$ breaths per min

Temperature $<36^{\circ} \mathrm{C}$

Altered mental status

Arterial oxyhaemoglobin saturation $<90 \%$
Age in years

+10 points

+30 points

+10 points

+10 points

+20 points

+30 points

+20 points

+20 points

+20 points
+60 points
1 point (if age $>80$ years)

1 point

1 point

1 point

1 point

$-$

$-$

1 point

Risk strata ${ }^{a}$

\section{Class I: $\leqslant 65$ points}

very low 30 day mortality risk (0-1.6\%)

Class II: $66-85$ points

low mortality risk (1.7-3.5\%)

Class III: 86-105 points

moderate mortality risk (3.2-7.1\%)

Class IV: 106-125 points

high mortality risk (4.0-11.4\%)

Class V: >125 points

very high mortality risk (10.0-24.5\%)
0 points

30 day mortality risk $1.0 \%$ (95\% Cl 0.0-2.1\%)

$\geqslant 1$ point(s)

30 day mortality risk $10.9 \%$

(95\% Cl 8.5-13.2\%)

BP: blood pressure; b.p.m.: beats per minute; $\mathrm{Cl}$ : confidence interval. ${ }^{\mathrm{a} B a s e d}$ on the sum of points.

\subsection{Prognostic assessment strategy}

The classification of PE severity and the risk of early (in-hospital or 30 day) death is summarized in table 8 . Risk assessment of acute PE begins upon suspicion of the disease and initiation of the diagnostic workup. At this early stage, it is critical to identify patients with (suspected) high-risk PE. This clinical setting necessitates an emergency diagnostic algorithm (figure 4) and immediate referral for reperfusion treatment, as explained in section 7, and displayed in figure 6 and supplementary figure 1. Testing for laboratory biomarkers such as cardiac troponins or natriuretic peptides is not necessary for immediate therapeutic decisions in patients with high-risk PE.

In the absence of haemodynamic instability at presentation, further risk stratification of PE is recommended, as it has implications for early discharge versus hospitalization or monitoring of the patient (explained in section 7). Table 8 provides an overview of the clinical, imaging, and laboratory parameters used to distinguish intermediate- and low-risk PE. The PESI is-in its original or simplified form-the most extensively validated and most broadly used clinical score to date, as it integrates baseline indicators of the severity of the acute PE episode with aggravating conditions and the comorbidity of the patient. Overall, a PESI of class I-II or an SPESI of 0 is a reliable predictor of low-risk PE.

In addition to clinical parameters, patients in the intermediate-risk group who display evidence of both RV dysfunction (on echocardiography or CTPA) and elevated cardiac biomarker levels in the circulation (particularly a positive cardiac troponin test) are classified into the intermediate-high-risk category. As will be discussed in more detail in section 7 , close monitoring is recommended in these cases to permit the early detection of haemodynamic decompensation or collapse, and consequently the need for rescue reperfusion therapy [179]. Patients in whom the RV appears normal on echocardiography or CTPA, and/or who have normal cardiac biomarker levels, belong to the intermediate-low-risk category. As an alternative approach, use of further prognostic scores combining clinical, imaging, and laboratory parameters may be considered to semi-quantitatively assess the severity of the PE episode, and distinguish intermediate-high-risk and intermediate-low-risk PE. Supplementary table 4 lists the scores most frequently investigated for this purpose in observational (cohort) studies; however, none of them has been used in RCTs to date.

A recent meta-analysis included 21 cohort studies with a total of 3295 patients with "low-risk" PE based on a PESI of I-II or an sPESI of 0 [234]. Overall, 34\% (95\% CI 30-39\%) of them were reported to have signs of RV dysfunction on echocardiography or CTPA. Data on early mortality were provided in seven studies (1597 patients) and revealed an OR of 4.19 (95\% CI 1.39-12.58) for death from any cause in the presence of RV dysfunction; elevated cardiac troponin levels were associated with a comparable magnitude of risk elevation [234]. 
TABLE 8 Classification of pulmonary embolism severity and the risk of early (in-hospital or 30 dayl death

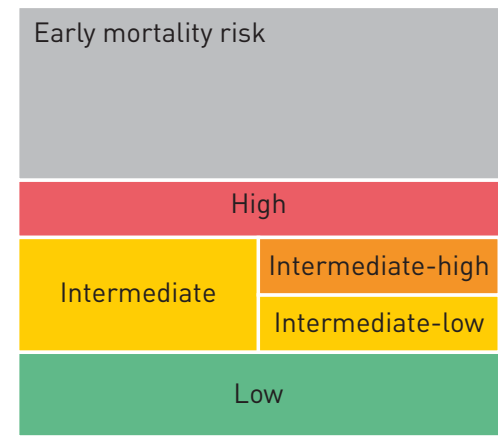

\begin{tabular}{|c|c|c|c|}
\hline \multicolumn{4}{|c|}{ Indicators of risk } \\
\hline $\begin{array}{l}\text { Haemodynamic } \\
\text { instabilitya }\end{array}$ & $\begin{array}{l}\text { Clinical parameters of PE } \\
\text { severity and/or comorbidity: } \\
\text { PESI class III-V or sPESI } \geqslant \mid\end{array}$ & $\begin{array}{l}\text { RV dysfunction } \\
\text { on TTE or CTPA }\end{array}$ & $\begin{array}{l}\text { Elevated cardiac } \\
\text { troponin levelsc }\end{array}$ \\
\hline+ & $1+)^{d}$ & + & $1+1$ \\
\hline- & $+e$ & + & + \\
\hline - & $+e$ & \multicolumn{2}{|c|}{ One lor nonel positive } \\
\hline- & - & - & $\begin{array}{l}\text { Assessment optional; } \\
\text { if assessed, negative }\end{array}$ \\
\hline
\end{tabular}

BP: blood pressure; CTPA: computed tomography pulmonary angiography; H-FABP:heart-type fatty acid-binding protein; NT-proBNP N-terminal pro B-type natriuretic peptide; PE: pulmonary embolism; PESI: Pulmonary Embolism Severity Index; RV: right ventricular; sPESI: simplified Pulmonary Embolism Severity Index; TTE: transthoracic echocardiogram. ${ }^{a}$ One of the following clinical presentations (table 4): cardiac arrest, obstructive shock (systolic BP $<90 \mathrm{mmHg}$ or vasopressors required to achieve a BP $\geqslant 90 \mathrm{mmHg}$ despite an adequate filling status, in combination with end-organ hypoperfusion), or persistent hypotension (systolic BP $<90 \mathrm{mmHg}$ or a systolic BP drop $\geqslant 40 \mathrm{mmHg}$ for $>15 \mathrm{~min}$, not caused by new-onset arrhythmia, hypovolaemia, or sepsis). ${ }^{\mathrm{b}}$ Prognostically relevant imaging (TTE or CTPA) findings in patients with acute $\mathrm{PE}$, and the corresponding cut-off levels, are graphically presented in figure 3 , and their prognostic value is summarized in supplementary table 3 . ' Elevation of further laboratory biomarkers, such as NT-proBNP $\geqslant 600 \mathrm{ng} / \mathrm{L}, \mathrm{H}-\mathrm{FABP} \geqslant 6 \mathrm{ng} / \mathrm{mL}$, or copeptin $\geqslant 24 \mathrm{pmol}$ $\mathrm{L}$, may provide additional prognostic information. These markers have been validated in cohort studies but they have not yet been used to guide treatment decisions in randomized controlled trials. ${ }^{\mathrm{d} H a e m o d y n a m i c ~ i n s t a b i l i t y, ~ c o m b i n e d ~ w i t h ~ P E ~ c o n f i r m a t i o n ~ o n ~ C T P A ~ a n d / o r ~ e v i d e n c e ~ o f ~ R V ~}$ dysfunction on TTE, is sufficient to classify a patient into the high-risk PE category. In these cases, neither calculation of the PESI nor measurement of troponins or other cardiac biomarkers is necessary. ${ }^{\mathrm{e}}$ Signs of RV dysfunction on TTE (or CTPA) or elevated cardiac biomarker levels may be present, despite a calculated PESI of I-II or an SPESI of 0 [234]. Until the implications of such discrepancies for the management of PE are fully understood, these patients should be classified into the intermediate-risk category.

Early all-cause mortality rates (1.8\% for RV dysfunction and 3.8\% for elevated troponin levels [234]) were in the lower range of those previously reported for patients with intermediate-risk PE [235]. Until the clinical implications of such discrepancies are clarified, patients with signs of RV dysfunction or elevated cardiac biomarkers, despite a low PESI or an sPESI of 0, should be classified into the intermediate-low-risk category.

\subsection{Recommendations for prognostic assessment}

\section{Recommendations}

Initial risk stratification of suspected or confirmed PE, based on the presence of haemodynamic instability, is recommended to identify patients at high risk of early mortality $[218,219,235]$.

In patients without haemodynamic instability, further stratification of patients with acute $\mathrm{PE}$ into intermediate- and low-risk categories is recommended [179, 218, 219, 235].

In patients without haemodynamic instability, use of clinical prediction rules integrating PE severity and comorbidity, preferably the PESI or SPESI, should be considered for risk assessment in the acute phase of PE $[178,226,229]$.

Assessment of the RV by imaging methodsc or laboratory biomarkersd should be considered, even in the presence of a low PESI or a negative SPESI [234].

In patients without haemodynamic instability, use of validated scores combining clinical, imaging, and laboratory PE-related prognostic factors may be considered to further stratify the severity of the acute PE episode [218-223].

\section{Class $^{a}$ Level $^{b}$}

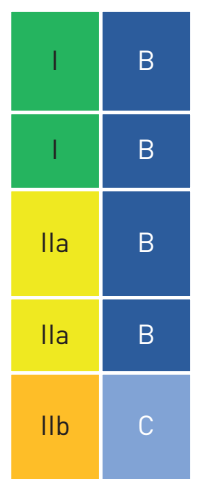

PE: pulmonary embolism; PESI: Pulmonary Embolism Severity Index; RV: right ventricle; sPESI: simplified Pulmonary Embolism Severity Index. ${ }^{a}$ Class of recommendation. ${ }^{b}$ Level of evidence. ${ }^{\mathrm{c}}$ Transthoracic echocardiography or computed tomography pulmonary angiography. ${ }^{\mathrm{d} C a r d i a c}$ troponins or natriuretic peptides.

\section{Treatment in the acute phase \\ 6.1. Haemodynamic and respiratory support \\ 6.1.1. Oxygen therapy and ventilation}

Hypoxaemia is one of the features of severe PE, and is mostly due to the mismatch between ventilation and perfusion. Administration of supplemental oxygen is indicated in patients with $\mathrm{PE}$ and $\mathrm{S}_{\mathrm{aO}_{2}}<90 \%$. Severe hypoxaemia/respiratory failure that is refractory to conventional oxygen supplementation could be 


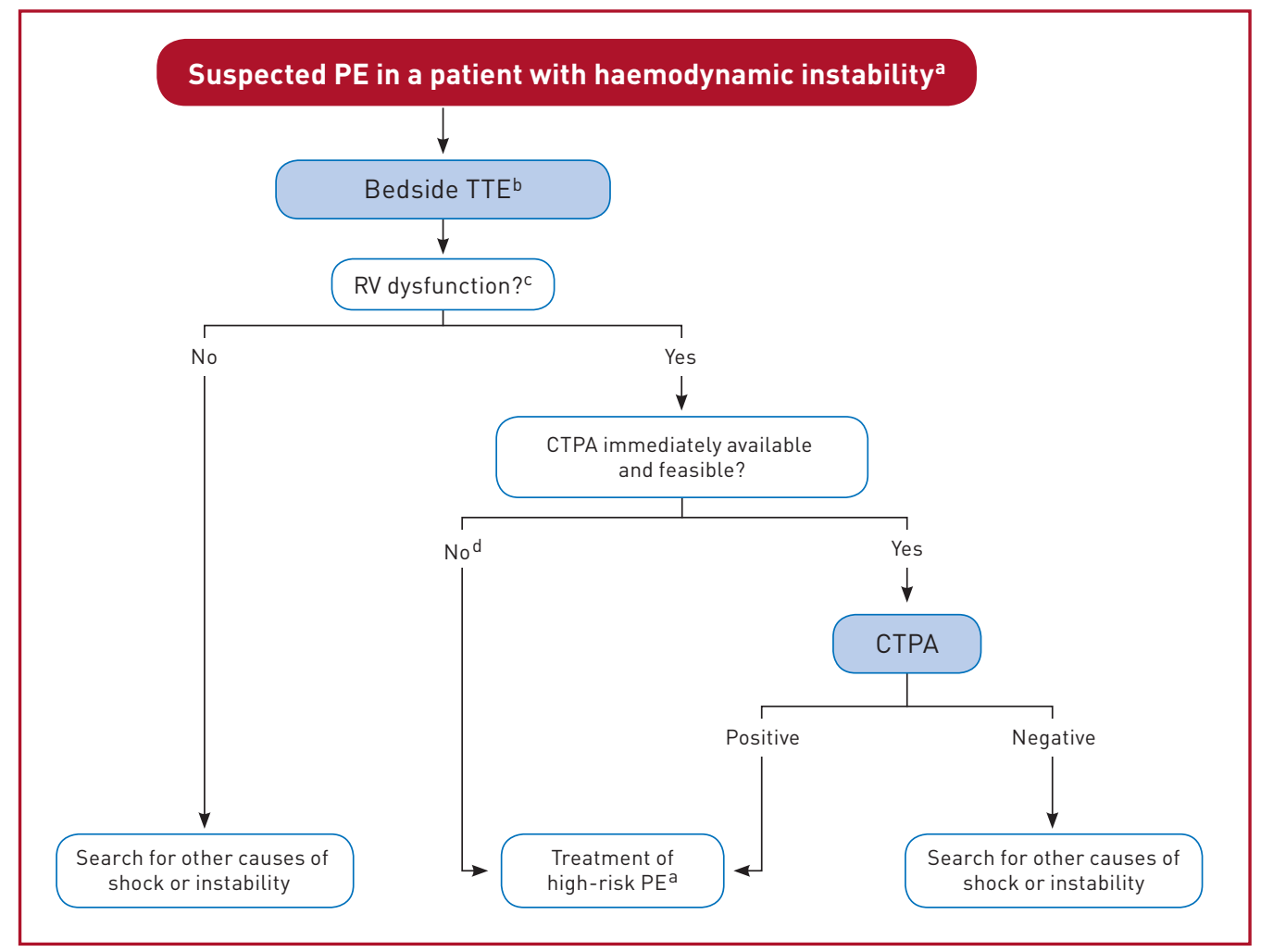

FIGURE 4 Diagnostic algorithm for patients with suspected high-risk pulmonary embolism presenting with haemodynamic instability. CTPA: computed tomography pulmonary angiography; CUS: compression ultrasonography; DVT: deep vein thrombosis; LV: left ventricle; PE: pulmonary embolism; RV: right ventricle;

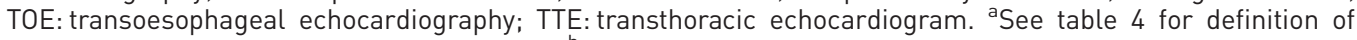
haemodynamic instability and high-risk PE. ${ }^{b}$ Ancillary bedside imaging tests may include TOE, which may detect emboli in the pulmonary artery and its main branches; and bilateral venous CUS, which may confirm DVT and thus VTE. "In the emergency situation of suspected high-risk PE, this refers mainly to a RV/LV diameter ratio $>1.0$; the echocardiographic findings of RV dysfunction, and the corresponding cut-off levels, are graphically presented in figure 3 , and their prognostic value summarized in supplementary table 3 . 'Includes the cases in which the patient's condition is so critical that it only allows bedside diagnostic tests. In such cases, echocardiographic findings of RV dysfunction confirm high-risk PE and emergency reperfusion therapy is recommended.

explained by right-to-left shunt through a patent foramen ovale or atrial septal defect [67]. Further oxygenation techniques should also be considered, including high-flow oxygen (i.e. a high-flow nasal cannula) $[236,237]$ and mechanical ventilation (non-invasive or invasive) in cases of extreme instability (i.e. cardiac arrest), taking into consideration that correction of hypoxaemia will not be possible without simultaneous pulmonary reperfusion.

Patients with RV failure are frequently hypotensive or are highly susceptible to the development of severe hypotension during induction of anaesthesia, intubation, and positive-pressure ventilation. Consequently, intubation should be performed only if the patient is unable to tolerate or cope with non-invasive ventilation. When feasible, non-invasive ventilation or oxygenation through a high-flow nasal cannula should be preferred; if mechanical ventilation is used, care should be taken to limit its adverse haemodynamic effects. In particular, positive intrathoracic pressure induced by mechanical ventilation may reduce venous return and worsen low $\mathrm{CO}$ due to $\mathrm{RV}$ failure in patients with high-risk PE; therefore, positive end-expiratory pressure should be applied with caution. Tidal volumes of approximately $6 \mathrm{~mL} / \mathrm{kg}$ lean body weight should be used in an attempt to keep the end-inspiratory plateau pressure $<30 \mathrm{~cm}$ $\mathrm{H}_{2} \mathrm{O}$. If intubation is needed, anaesthetic drugs more prone to cause hypotension should be avoided for induction.

\subsubsection{Pharmacological treatment of acute right ventricular failure}

Acute RV failure with resulting low systemic output is the leading cause of death in patients with high-risk $\mathrm{PE}$. The principles of acute right heart failure management have been reviewed in a statement from the Heart Failure Association and the Working Group on Pulmonary Circulation and Right Ventricular Function of the ESC [68]. An overview of the current treatment options for acute RV failure is provided in table 9. 
TABLE 9 Treatment of right ventricular failure in acute high-risk pulmonary embolism

Strategy Properties and use Caveats
Volume optimization
Cautious volume loading, saline, or Ringer's lactate,
$\quad \leqslant 500 \mathrm{~mL}$ over $15-30 \mathrm{~min}$

\section{Vasopressors and inotropes}

Norepinephrine, $0.2-1.0 \mu \mathrm{g} / \mathrm{kg} / \mathrm{min}^{\mathrm{a}}$ [240]

Dobutamine, 2-20 $\mu \mathrm{g} / \mathrm{kg} / \mathrm{min}[241]$

\section{Mechanical circulatory support}

Veno-arterial ECMO/extracorporeal life support $[251,252,258]$
Consider in patients with normal-low central venous pressure (due, for example, to concomitant hypovolaemial

Increases RV inotropy and systemic BP, promotes positive ventricular interactions, and restores coronary perfusion gradient

Increases RV inotropy, lowers filling pressures

Rapid short-term support combined with oxygenator
Volume loading can over-distend the RV, worsen ventricular interdependence, and reduce $\mathrm{CO}$ [239]

Excessive vasoconstriction may worsen tissue perfusion

May aggravate arterial hypotension if used alone, without a vasopressor; may trigger or aggravate arrhythmias

Complications with use over longer periods ( $>5-10$ days), including bleeding and infections; no clinical benefit unless combined with surgical embolectomy; requires an experienced team

CO: cardiac output; BP: blood pressure; ECMO: extracorporeal membrane oxygenation; RV: right ventricle/ventricular. ${ }^{a}$ Epinephrine is used in cardiac arrest.

If the central venous pressure is low, modest $(\leqslant 500 \mathrm{~mL})$ fluid challenge can be used as it may increase the cardiac index in patients with acute PE [238]. However, volume loading has the potential to over-distend the RV and ultimately cause a reduction in systemic CO [239]. Experimental studies suggest that aggressive volume expansion is of no benefit and may even worsen RV function [240]. Cautious volume loading may be appropriate if low arterial pressure is combined with an absence of elevated filling pressures. Assessment of central venous pressure by ultrasound imaging of the IVC (a small and/or collapsible IVC in the setting of acute high-risk PE indicates low volume status) or, alternatively, by central venous pressure monitoring may help guide volume loading. If signs of elevated central venous pressure are observed, further volume loading should be withheld.

Use of vasopressors is often necessary, in parallel with (or while waiting for) pharmacological, surgical, or interventional reperfusion treatment. Norepinephrine can improve systemic haemodynamics by bringing about an improvement in ventricular systolic interaction and coronary perfusion, without causing a change in PVR [240]. Its use should be limited to patients in cardiogenic shock. Based on the results of a small series, the use of dobutamine may be considered for patients with PE, a low cardiac index, and normal BP; however, raising the cardiac index may aggravate the ventilation/perfusion mismatch by further redistributing flow from (partly) obstructed to unobstructed vessels [241]. Although experimental data suggest that levosimendan may restore RV-pulmonary arterial coupling in acute PE by combining pulmonary vasodilation with an increase in RV contractility [242], no evidence of clinical benefit is available.

Vasodilators decrease PAP and PVR, but may worsen hypotension and systemic hypoperfusion due to their lack of specificity for the pulmonary vasculature after systemic [intravenous (i.v.)] administration. Although small clinical studies have suggested that inhalation of nitric oxide may improve the haemodynamic status and gas exchange of patients with PE [243-245], no evidence for its clinical efficacy or safety is available to date [246].

\subsubsection{Mechanical circulatory support and oxygenation}

The temporary use of mechanical cardiopulmonary support, mostly with veno-arterial extracorporeal membrane oxygenation (ECMO), may be helpful in patients with high-risk PE, and circulatory collapse or cardiac arrest. Survival of critically ill patients has been described in a number of case series [247-252], but no RCTs testing the efficacy and safety of these devices in the setting of high-risk PE have been conducted to date. Use of ECMO is associated with a high incidence of complications, even when used for short periods, and the results depend on the experience of the centre as well as patient selection. The increased risk of bleeding related to the need for vascular access should be considered, particularly in patients undergoing thrombolysis. At present, the use of ECMO as a stand-alone technique with anticoagulation is controversial $[247,252]$ and additional therapies, such as surgical embolectomy, have to be considered. 
A few cases suggesting good outcomes with use of the Impella ${ }^{\star}$ catheter in patients in shock caused by acute PE have been reported [253, 254].

\subsubsection{Advanced life support in cardiac arrest}

Acute PE is part of the differential diagnosis of cardiac arrest with non-shockable rhythm against a background of pulseless electrical activity. In cardiac arrest presumably caused by acute PE, current guidelines for advanced life support should be followed [255, 256]. The decision to treat for acute PE must be taken early, when a good outcome is still possible. Thrombolytic therapy should be considered; once a thrombolytic drug is administered, cardiopulmonary resuscitation should be continued for at least 60-90 min before terminating resuscitation attempts [257].

\subsection{Initial anticoagulation}

\subsubsection{Parenteral anticoagulation}

In patients with high or intermediate clinical probability of PE (see section 4), anticoagulation should be initiated while awaiting the results of diagnostic tests. This is usually done with subcutaneous, weight-adjusted low-molecular weight heparin (LMWH) or fondaparinux (supplementary table 5), or i.v. unfractionated heparin (UFH). Based on pharmacokinetic data (supplementary table 6) [259], an equally rapid anticoagulant effect can also be achieved with a non-vitamin $\mathrm{K}$ antagonist oral anticoagulant (NOAC), and phase III clinical trials have demonstrated the non-inferior efficacy of a single-oral drug anticoagulation strategy using higher doses of apixaban for 7 days or rivaroxaban for 3 weeks [259-261].

LMWH and fondaparinux are preferred over UFH for initial anticoagulation in PE, as they carry a lower risk of inducing major bleeding and heparin-induced thrombocytopenia [262-265]. Neither LMWH nor fondaparinux need routine monitoring of anti-Xa levels. Use of UFH is nowadays largely restricted to patients with overt haemodynamic instability or imminent haemodynamic decompensation in whom primary reperfusion treatment will be necessary. UFH is also recommended for patients with serious renal impairment [creatinine clearance $(\mathrm{CrCl}) \leqslant 30 \mathrm{~mL} / \mathrm{min}$ ] or severe obesity. If LMWH is prescribed in patients with $\mathrm{CrCl} 15-30 \mathrm{~mL} / \mathrm{min}$, an adapted dosing scheme should be used. The dosing of UFH is adjusted based on the activated partial thromboplastin time (supplementary table 7) [266].

\subsubsection{Non-vitamin K antagonist oral anticoagulants}

NOACs are small molecules that directly inhibit one activated coagulation factor, which is thrombin for dabigatran and factor Xa for apixaban, edoxaban, and rivaroxaban. The characteristics of NOACs used in the treatment of acute $\mathrm{PE}$ are summarized in supplementary table 6. Owing to their predictable bioavailability and pharmacokinetics, NOACs can be given at fixed doses without routine laboratory monitoring. Compared with vitamin $\mathrm{K}$ antagonists (VKAs), there are fewer interactions when NOACs are given concomitantly with other drugs [259]. In the phase III VTE trials, the dosages of dabigatran, rivaroxaban, and apixaban were not reduced in patients with mild-moderate renal dysfunction $(\mathrm{CrCl}$ 30-60 mL/min), whereas edoxaban was given at a $30 \mathrm{mg}$ dose in these patients. Patients with $\mathrm{CrCl}<25 \mathrm{~mL} / \mathrm{min}$ were excluded from the trials testing apixaban, whereas patients with $\mathrm{CrCl}<30 \mathrm{~mL} / \mathrm{min}$ were excluded from those investigating rivaroxaban, edoxaban, and dabigatran (supplementary table 8).

Phase III trials on the treatment of acute VTE (supplementary table 8), as well as those on extended treatment beyond the first 6 months (see section 8), demonstrated the non-inferiority of NOACs compared with the combination of LMWH with VKA for the prevention of symptomatic or lethal VTE recurrence, along with significantly reduced rates of major bleeding [267]. The different drug regimens tested in these trials are displayed in supplementary table 8 . In a meta-analysis, the incidence rate of the primary efficacy outcome was $2.0 \%$ for NOAC-treated patients and 2.2\% for VKA-treated patients [relative risk (RR) 0.88 , 95\% CI 0.74-1.05] [268]. Major bleeding occurred in $1.1 \%$ of NOAC-treated patients and $1.7 \%$ of VKA-treated patients for an RR of 0.60 (95\% CI 0.41-0.88). Compared with VKA-treated patients, critical site major bleeding occurred less frequently in NOAC-treated patients (RR 0.38, 95\% CI 0.23- 0.62); in particular, there was a significant reduction in intracranial bleeding (RR 0.37, 95\% CI 0.21-0.68) and in fatal bleeding (RR 0.36, 95\% CI 0.15-0.87) with NOACs compared with VKAs [268].

Suggestions for the anticoagulation management of PE in specific clinical situations, for which conclusive evidence is lacking, are presented in supplementary table 9.

Practical guidance for clinicians regarding the handling of NOACs and the management of emergency situations related to their use are regularly updated by the European Heart Rhythm Association [259].

\subsubsection{Vitamin K antagonists}

VKAs have been the gold standard in oral anticoagulation for more than 50 years. When VKAs are used, anticoagulation with UFH, LMWH, or fondaparinux should be continued in parallel with the oral anticoagulant for 
$\geqslant 5$ days and until the international normalized ratio (INR) value has been 2.0-3.0 for 2 consecutive days. Warfarin may be started at a dose of $10 \mathrm{mg}$ in younger (e.g. aged $<60$ years) otherwise healthy patients and at a dose $\leqslant 5 \mathrm{mg}$ in older patients [269]. The daily dose is adjusted according to the INR over the next 5-7 days, aiming for an INR level of 2.0-3.0. Pharmacogenetic testing may increase the precision of warfarin dosing [270, 271]. When used in addition to clinical parameters, pharmacogenetic testing improves anticoagulation control and may be associated with a reduced risk of bleeding, but does not reduce the risk of thromboembolic events or mortality [272].

The implementation of a structured anticoagulant service (most commonly, anticoagulant clinics) appears to be associated with increased time in the therapeutic range and improved clinical outcome, compared with control of anticoagulation by the general practitioner [273, 274]. Finally, in patients who are selected and appropriately trained, self-monitoring of VKA is associated with fewer thrombo-embolic events and increased time in the therapeutic range compared with usual care [275].

\subsection{Reperfusion treatment}

\subsubsection{Systemic thrombolysis}

Thrombolytic therapy leads to faster improvements in pulmonary obstruction, PAP, and PVR in patients with PE, compared with UFH alone; these improvements are accompanied by a reduction in RV dilation on echocardiography [276-279]. The greatest benefit is observed when treatment is initiated within $48 \mathrm{~h}$ of symptom onset, but thrombolysis can still be useful in patients who have had symptoms for 6-14 days [280]. Unsuccessful thrombolysis, as judged by persistent clinical instability and unchanged RV dysfunction on echocardiography after $36 \mathrm{~h}$, has been reported in $8 \%$ of high-risk PE patients [281].

A meta-analysis of thrombolysis trials that included (but were not confined to) patients with high-risk PE, defined mainly as the presence of cardiogenic shock, indicated a significant reduction in the combined outcome of mortality and recurrent PE (supplementary table 10). This was achieved with a 9.9\% rate of severe bleeding and a $1.7 \%$ rate of intracranial haemorrhage [282].

In normotensive patients with intermediate-risk PE, defined as the presence of RV dysfunction and elevated troponin levels, the impact of thrombolytic treatment was investigated in the Pulmonary Embolism Thrombolysis (PEITHO) trial [179]. Thrombolytic therapy was associated with a significant reduction in the risk of haemodynamic decompensation or collapse, but this was paralleled by an increased risk of severe extracranial and intracranial bleeding [179]. In the PEITHO trial, 30 day death rates were low in both treatment groups, although meta-analyses have suggested a reduction in PE-related and overall mortality of as much as $50-60 \%$ following thrombolytic treatment in the intermediate-risk category (supplementary table 10) [282, 283].

The approved regimens and doses of thrombolytic agents for PE, as well as the contraindications to this type of treatment, are shown in table 10. Accelerated i.v. administration of recombinant tissue-type plasminogen activator ( $\mathrm{rtPA} ; 100 \mathrm{mg}$ over $2 \mathrm{~h}$ ) is preferable to prolonged infusions of first-generation thrombolytic agents (streptokinase and urokinase). Preliminary reports on the efficacy and safety of reduced-dose rtPA $[284,285]$ need confirmation by solid evidence before any recommendations can be made in this regard. UFH may be administered during continuous infusion of alteplase, but should be discontinued during infusion of streptokinase or urokinase [65]. Reteplase [286], desmoteplase [287], or tenecteplase [179, $278,279]$ have also been investigated; at present, none of these agents are approved for use in acute PE.

It remains unclear whether early thrombolysis for (intermediate- or high-risk) acute PE has an impact on clinical symptoms, functional limitation, or CTEPH at long-term follow-up. A small randomized trial of 83 patients suggested that thrombolysis might improve functional capacity at 3 months compared with anticoagulation alone [278]. In the PEITHO trial [179], mild persisting symptoms, mainly dyspnoea, were present in $33 \%$ of the patients at long-term (at $41.6 \pm 15.7$ months) clinical follow-up [288]. However, the majority of patients ( $85 \%$ in the tenecteplase arm and $96 \%$ in the placebo arm) had a low or intermediate probability-based on the ESC Guidelines definition [289]—of persisting or new-onset PH at echocardiographic follow-up [288]. Consequently, the findings of this study do not support a role for thrombolysis with the aim of preventing long-term sequelae (section 10) after intermediate-risk PE, although they are limited by the fact that clinical follow-up was available for only $62 \%$ of the study population.

\subsubsection{Percutaneous catheter-directed treatment}

Mechanical reperfusion is based on the insertion of a catheter into the pulmonary arteries via the femoral route. Different types of catheters (summarized in supplementary table 11) are used for mechanical fragmentation, thrombus aspiration, or more commonly a pharmacomechanical approach combining mechanical or ultrasound fragmentation of the thrombus with in situ reduced-dose thrombolysis.

Most knowledge about catheter-based embolectomy is derived from registries and pooled results from case series $[290,291]$. The overall procedural success rates (defined as haemodynamic stabilization, correction of hypoxia, 
TABLE 10 Thrombolytic regimens, doses, and contraindications

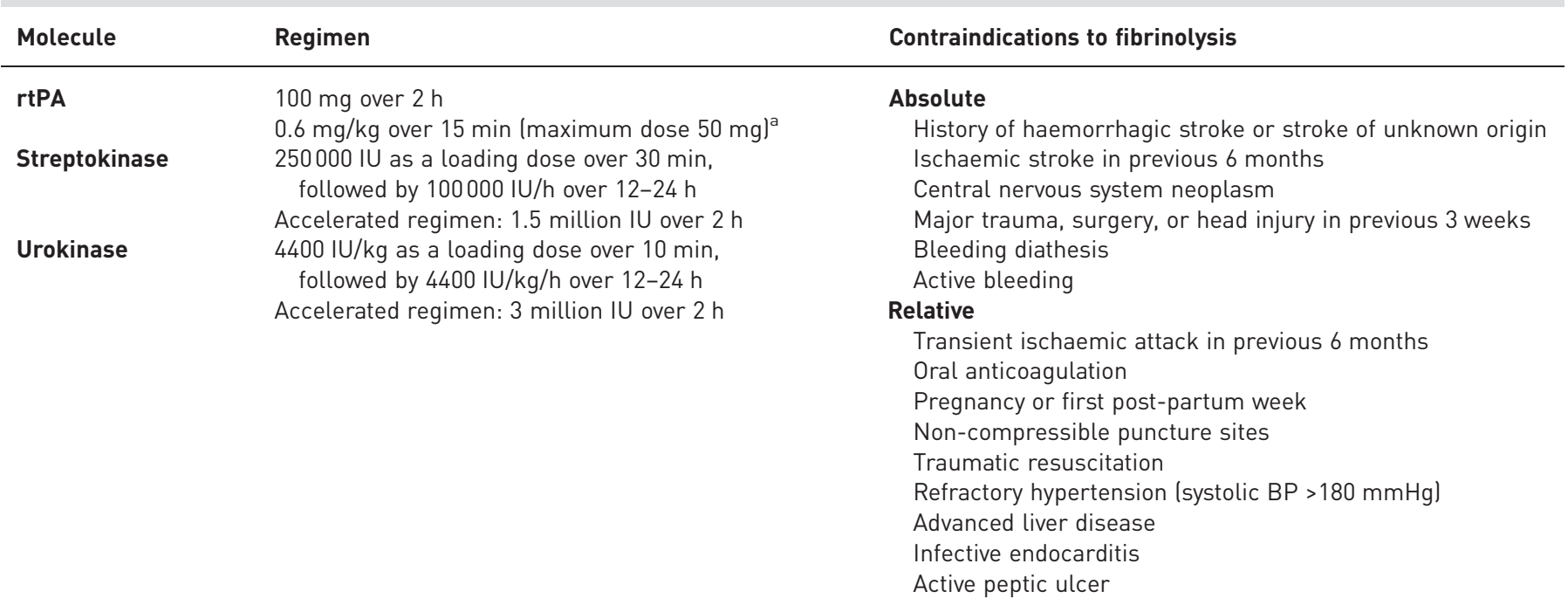

BP: blood pressure; IU: international units; rtPA, recombinant tissue-type plasminogen activator. ${ }^{\text {aThis }}$ is the accelerated regimen for rtPA in pulmonary embolism; it is not officially approved, but it is sometimes used in extreme haemodynamic instability such as cardiac arrest.

and survival to hospital discharge) of percutaneous catheter-based therapies reported in these studies have reached 87\% [292]; however, these results may be subject to publication bias. One RCT compared conventional heparin-based treatment and a catheter-based therapy combining ultrasound-based clot fragmentation with low-dose in situ thrombolysis in 59 patients with intermediate-risk PE. In that study, ultrasound-assisted thrombolysis was associated with a larger decrease in the RV/LV diameter ratio at $24 \mathrm{~h}$, without an increased risk of bleeding [293]. Data from two prospective cohort studies [294, 295] and a registry [296], with a total of 352 patients, support the improvement in RV function, lung perfusion, and PAP in patients with intermediate- or high-risk PE using this technique. Intracranial haemorrhage was rare, although the rate of Global Utilization of Streptokinase and Tissue Plasminogen Activator for Occluded Coronary Arteries (GUSTO) severe and moderate bleeding complications was $10 \%$ in one of these cohorts [294]. These results should be interpreted with caution, considering the relatively small numbers of patients treated, the lack of studies directly comparing catheter-directed with systemic thrombolytic therapy, and the lack of data from RCTs on clinical efficacy outcomes.

\subsubsection{Surgical embolectomy}

Surgical embolectomy in acute PE is usually carried out with cardiopulmonary bypass, without aortic cross-clamping and cardioplegic cardiac arrest, followed by incision of the two main pulmonary arteries with the removal or suction of fresh clots. Recent reports have indicated favourable surgical results in high-risk PE, with or without cardiac arrest, and in selected cases of intermediate-risk PE [297-300]. Among 174322 patients hospitalized between 1999 and 2013 with a diagnosis of PE in New York state, survival and recurrence rates were compared between patients who underwent thrombolysis $(n=1854)$ or surgical embolectomy $(n=257)$ as first-line therapy [297]. Overall, there was no difference between the two types of reperfusion treatment regarding 30 day mortality (15 and 13\%, respectively), but thrombolysis was associated with a higher risk of stroke and re-intervention at 30 days. No difference was found in terms of 5 year actuarial survival, but thrombolytic therapy was associated with a higher rate of recurrent PE requiring readmission compared with surgery (7.9 versus $2.8 \%$ ). However, the two treatments were not randomly allocated in this observational retrospective study, and the patients referred for surgery may have been selected. An analysis of the Society of Thoracic Surgery Database with multicentre data collection, including 214 patients submitted for surgical embolectomy for high- $(n=38)$ or intermediate-risk $(n=176)$ PE, revealed an in-hospital mortality rate of $12 \%$, with the worst outcome (32\%) in the group experiencing pre-operative cardiac arrest [299].

Recent experience appears to support combining ECMO with surgical embolectomy, particularly in patients with high-risk PE with or without the need for cardiopulmonary resuscitation. Among patients who presented with intermediate-risk PE $(n=28)$, high-risk PE without cardiac arrest $(n=18)$, and PE with cardiac arrest $(n=9)$, the in-hospital and 1 year survival rates were 93 and $91 \%$, respectively [300].

\subsection{Multidisciplinary pulmonary embolism teams}

The concept of multidisciplinary rapid-response teams for the management of "severe" (high-risk and selected cases of intermediate-risk) PE emerged in the USA, with increasing acceptance by the medical 
community and implementation in hospitals in Europe and worldwide. Set-up of PE response teams (PERTs) is encouraged, as they address the needs of modern systems-based healthcare [301]. A PERT brings together a team of specialists from different disciplines including, for example, cardiology, pulmonology, haematology, vascular medicine, anaesthesiology/intensive care, cardiothoracic surgery, and (interventional) radiology. The team convenes in real time (face-to-face or via web conference) to enhance clinical decision-making. This allows the formulation of a treatment plan and facilitates its immediate implementation [301]. The exact composition and operating mode of a PERT are not fixed, depending on the resources and expertise available in each hospital for the management of acute PE.

\subsection{Vena cava filters}

The aim of vena cava interruption is to mechanically prevent venous clots from reaching the pulmonary circulation. Most devices in current use are inserted percutaneously and can be retrieved after several weeks or months, or left in place over the long-term, if needed. Potential indications include VTE and absolute contraindication to anticoagulant treatment, recurrent PE despite adequate anticoagulation, and primary prophylaxis in patients with a high risk of VTE. Other potential indications for filter placement, including free-floating thrombi, have not been confirmed in patients without contraindications to therapeutic anticoagulation.

Only two phase III randomized trials have compared anticoagulation with or without vena cava interruption in patients with proximal DVT, with or without associated PE [302-304]. In the Prevention of Recurrent Pulmonary Embolism by Vena Cava Interruption (PREPIC) study, insertion of a permanent vena cava filter was associated with a significant reduction in the risk of recurrent PE and a significant increase in the risk of DVT, without a significant difference in the risk of recurrent VTE or death [303, 304]. The PREPIC-2 trial randomized 399 patients with PE and venous thrombosis to receive anticoagulant treatment, with or without a retrievable vena cava filter. In this study, the rate of recurrent VTE was low in both groups and did not differ between groups [302]. A systematic review and meta-analysis of published reports on the efficacy and safety of vena cava filters included 11 studies, with a total of 2055 patients who received a filter versus 2149 controls [305]. Vena cava filter placement was associated with a 50\% decrease in the incidence of PE and an $\sim 70 \%$ increase in the risk of DVT over time. Neither all-cause mortality nor PE-related mortality differed between patients with or without filter placement.

The broad indication for placement of a venous filter in patients with recent $(<1$ month) proximal DVT and an absolute contraindication to anticoagulant treatment is based mainly on the perceived high risk of recurrent $\mathrm{PE}$ in this setting, and the lack of other treatment options.

Complications associated with vena cava filters are common and can be serious. A systematic literature review revealed penetration of the venous wall in 1699 (19\%) of 9002 procedures; of these cases, 19\% showed adjacent organ involvement and $\geqslant 8 \%$ were symptomatic [306]. Lethal complications were rare (only two cases), but $5 \%$ of the patients required major interventions such as surgical removal of the filter, endovascular stent placement or embolization, endovascular retrieval of the permanent filter, or percutaneous nephrostomy or ureteral stent placement [306]. Further reported complications include filter fracture and/or embolization, and DVT occasionally extending up to the vena cava $[303,307,308]$.

\subsection{Recommendations for acute-phase treatment of high-risk pulmonary embolism ${ }^{a}$}

\section{Recommendations}

It is recommended that anticoagulation with UFH, including a weight-adjusted bolus injection, be initiated without delay in patients with high-risk PE.

Systemic thrombolytic therapy is recommended for high-risk PE [282].

Surgical pulmonary embolectomy is recommended for patients with high-risk $P E$, in whom thrombolysis is contraindicated or has failedd [281].

Percutaneous catheter-directed treatment should be considered for patients with high-risk $P E$, in whom thrombolysis is contraindicated or has failedd.

Norepinephrine and/or dobutamine should be considered in patients with high-risk PE.

ECMO may be considered, in combination with surgical embolectomy or catheter-directed treatment, in patients with PE and refractory circulatory collapse or cardiac arrest ${ }^{d}$ [252].
Class $^{b}$ Level $^{c}$

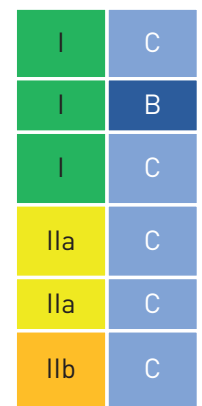

ECMO: extracorporeal membrane oxygenation; PE: pulmonary embolism; UFH: unfractionated heparin. ${ }^{a}$ See table 4 for definition of high-risk PE. After haemodynamic stabilization of the patient, continue with anticoagulation treatment as in intermediate- or low-risk PE (section 6.7). ${ }^{\mathrm{b}} \mathrm{Class}$ of recommendation.

${ }^{c}$ Level of evidence. ${ }^{d}$ If appropriate expertise and resources are available on-site. 


\subsection{Recommendations for acute-phase treatment of intermediate- or low-risk pulmonary embolism}

Recommendations

Initiation of anticoagulation

Initiation of anticoagulation is recommended without delay in patients with high or intermediate clinical probability of $\mathrm{PE}^{\mathrm{c}}$, while diagnostic workup is in progress.

If anticoagulation is initiated parenterally, LMWH or fondaparinux is recommended lover UFH) for most patients [262, 309-311].

When oral anticoagulation is started in a patient with PE who is eligible for a NOAC (apixaban, dabigatran, edoxaban, or rivaroxaban), a NOAC is recommended in preference to a VKA [260, 261, 312-314].

When patients are treated with a VKA, overlapping with parenteral anticoagulation is recommended until an INR of 2.5 (range 2.0-3.0) is reached [315, 316].

NOACs are not recommended in patients with severe renal impairment, during pregnancy and lactation, and in patients with antiphospholipid antibody syndrome [260, 261, 312-314].

\section{Reperfusion treatment}

Rescue thrombolytic therapy is recommended for patients with haemodynamic deterioration on anticoagulation treatment [282].

As an alternative to rescue thrombolytic therapy, surgical embolectomye or percutaneous catheter-directed treatmente should be considered for patients with haemodynamic deterioration on anticoagulation treatment.

Routine use of primary systemic thrombolysis is not recommended in patients with intermediate- or low-risk PEc,f [179].
Class $^{a}$ Level $^{b}$
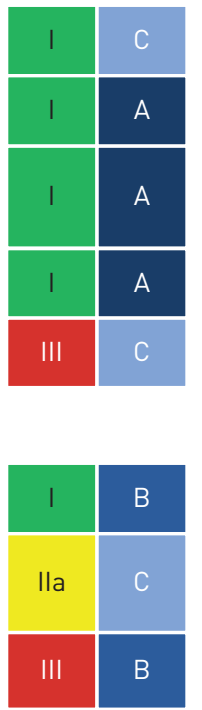

$\mathrm{CrCl}$ : creatinine clearance; INR: international normalized ratio; LMWH: low-molecular weight heparin; NOAC(s): non-vitamin $\mathrm{K}$ antagonist oral anticoagulant(s); PE: pulmonary embolism; UFH: unfractionated heparin; VKA: vitamin $\mathrm{K}$ antagonist. ${ }^{\mathrm{a}}$ Class of recommendation. ${ }^{\mathrm{b}}$ Level of evidence. ${ }^{\mathrm{c}} \mathrm{See}$ table 8 for definition of the PE severity and PE-related risk. ${ }^{\mathrm{d}}$ Dabigatran is not recommended in patients with $\mathrm{CrCl}<30 \mathrm{~mL} / \mathrm{min}$. Edoxaban should be given at a dose of $30 \mathrm{mg}$ once daily in patients with $\mathrm{CrCl}$ of $15-50 \mathrm{~mL} / \mathrm{min}$ and is not recommended in patients with $\mathrm{CrCl}<15 \mathrm{~mL} / \mathrm{min}$. Rivaroxaban and apixaban are to be used with caution in patients with $\mathrm{CrCl} 15-29 \mathrm{~mL} / \mathrm{min}$, and their use is not recommended in patients with $\mathrm{CrCl}<15 \mathrm{~mL} / \mathrm{min}$. elf appropriate expertise and resources are available on-site. ${ }^{\mathrm{f}}$ The risk-to-benefit ratios of surgical embolectomy or catheter-directed procedures have not yet been established in intermediate- or low-risk PE.

\subsection{Recommendations for multidisciplinary pulmonary embolism teams}

\section{Recommendations}

Class $^{\mathrm{a}}$ Level $^{\mathrm{b}}$

Set-up of a multidisciplinary team and a programme for the management of high- and lin selected cases) intermediate-risk PE should be considered, depending on the resources and expertise available in each hospital.

PE: pulmonary embolism. ${ }^{a}$ Class of recommendation. ${ }^{b}$ Level of evidence.

\subsection{Recommendations for inferior vena cava filters}

\section{Recommendations}

Class $^{\mathbf{a}}$ Level $^{\mathrm{b}}$

IVC filters should be considered in patients with acute PE and absolute contraindications to anticoagulation.

IVC filters should be considered in cases of PE recurrence despite therapeutic anticoagulation.

Routine use of IVC filters is not recommended [302-304]

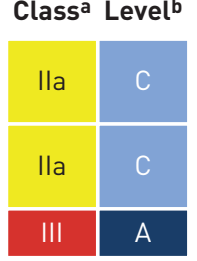

IVC: inferior vena cava; PE: pulmonary embolism. ${ }^{a}$ Class of recommendation. ${ }^{\mathrm{b}}$ Level of evidence.

\subsection{Recommendations for early discharge and home treatment}

\section{Recommendations}

Carefully selected patients with low-risk PE should be considered for early discharge and continuation of treatment at home, if proper outpatient care and anticoagulant treatment can be providedc $[178,206,317-319]$.

\section{Class ${ }^{a}$ Levelb}

Ila

PE: pulmonary embolism. ${ }^{a}$ Class of recommendation. ${ }^{b}$ Level of evidence. ${ }^{\mathrm{c}}$ See section 7 and figure 6 for further guidance on defining low-risk PE and decision-making. 


\section{Integrated risk-adapted diagnosis and management}

\subsection{Diagnostic strategies}

Various combinations of clinical assessments, plasma D-dimer measurements, and imaging tests have been proposed and validated for PE diagnosis. These strategies have been tested in patients presenting with suspected PE in the emergency department or during their hospital stay [101, 164, 171, 320], and more recently in the primary care setting [111]. Withholding of anticoagulation without adherence to evidence-based diagnostic strategies was associated with a significant increase in the number of VTE episodes and sudden cardiac death at 3 month follow-up [12]. The most straightforward diagnostic algorithms for suspected PE-with and without haemodynamic instability-are presented in figures 4 and 5, respectively. However, it is recognized that the diagnostic approach for suspected PE may vary, depending on the availability of, and expertise in, specific tests in various hospitals and clinical settings.

The diagnostic strategy for suspected acute PE in pregnancy is discussed in section 9.

\subsubsection{Suspected pulmonary embolism with haemodynamic instability}

The proposed strategy is shown in figure 4. The clinical probability is usually high and the differential diagnosis includes cardiac tamponade, acute coronary syndrome, aortic dissection, acute valvular

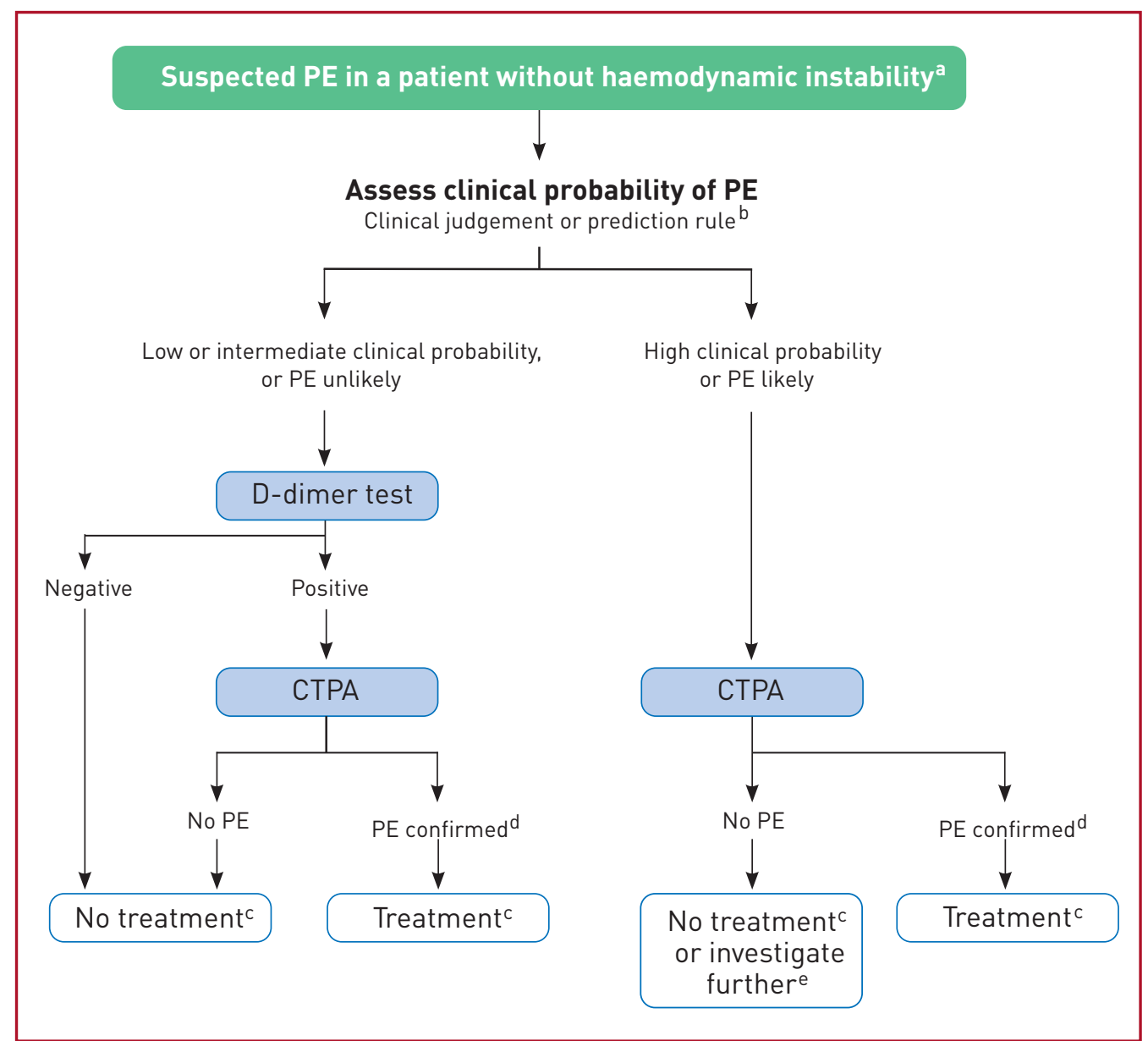

FIGURE 5 Diagnostic algorithm for patients with suspected pulmonary embolism without haemodynamic instability. CTPA: computed tomography pulmonary angiography/angiogram; PE: pulmonary embolism. ${ }^{\mathrm{a}}$ The proposed diagnostic strategy for pregnant women with suspected acute PE is discussed in section 9. ${ }^{\text {b}}$ Two alternative classification schemes may be used for clinical probability assessment, i.e. a three-level scheme (clinical probability defined as low, intermediate, or high) or a two-level scheme (PE unlikely or PE likely). When using a moderately sensitive assay, D-dimer measurement should be restricted to patients with low clinical probability or a PE-unlikely classification, while highly sensitive assays may also be used in patients with intermediate clinical probability of PE due to a higher sensitivity and negative predictive value. Note that plasma D-dimer measurement is of limited use in suspected PE occurring in hospitalized patients. ${ }^{c}$ Treatment refers to anticoagulation treatment for PE. ${ }^{d}$ CTPA is considered diagnostic of PE if it shows PE at the segmental or more proximal level. ${ }^{~}$ In case of a negative CTPA in patients with high clinical probability, investigation by further imaging tests may be considered before withholding PE-specific treatment. 
dysfunction, and hypovolaemia. The most useful initial test in this situation is bedside TTE, which will yield evidence of acute RV dysfunction if acute PE is the cause of the patient's haemodynamic decompensation. In a highly unstable patient, echocardiographic evidence of RV dysfunction is sufficient to prompt immediate reperfusion without further testing. This decision may be strengthened by the (rare) visualization of right heart thrombi $[155,157,321,322]$. Ancillary bedside imaging tests include TOE, which may allow direct visualization of thrombi in the pulmonary artery and its main branches, especially in patients with RV dysfunction. TOE should be cautiously performed in hypoxaemic patients. Moreover, bedside CUS can detect proximal DVT. As soon as the patient is stabilized using supportive treatment, final confirmation of the diagnosis by CT angiography should be sought.

For unstable patients admitted directly to the catheterization laboratory with suspected acute coronary syndrome, pulmonary angiography may be considered as a diagnostic procedure after the acute coronary syndrome has been excluded, provided that PE is a probable diagnostic alternative and particularly if percutaneous catheter-directed treatment is a therapeutic option.

7.1.2. Suspected pulmonary embolism without haemodynamic instability

7.1.2.1. Strategy based on computed tomographic pulmonary angiography

The proposed strategy based on CTPA is shown in figure 5. In patients admitted to the emergency department, measurement of plasma D-dimer is the logical first step following the assessment of clinical probability and allows PE to be ruled out in $\sim 30 \%$ of outpatients. D-dimer should not be measured in patients with a high clinical probability of $\mathrm{PE}$, owing to a low negative predictive value in this population [323]. It is also less useful in hospitalized patients because the number that needs to be tested to obtain a clinically relevant negative result is high.

In most centres, multidetector CTPA is the second-line test in patients with an elevated D-dimer level and the first-line test in patients with a high clinical probability of PE. CTPA is considered to be diagnostic of $\mathrm{PE}$ when it shows a clot at least at the segmental level of the pulmonary arterial tree. False-negative results of CTPA have been reported in patients with a high clinical probability of PE [115]; however, such discrepancies are infrequent and the 3 month thromboembolic risk was low in these patients [171]. Accordingly, both the necessity of performing further tests and the nature of these tests remain controversial in these clinical situations.

\subsubsection{Strategy based on ventilation/perfusion scintigraphy}

In hospitals in which V/Q scintigraphy is readily available, it is a valid option for patients with an elevated D-dimer and a contraindication to CTPA. Also, V/Q scintigraphy may be preferred over CTPA to avoid unnecessary radiation, particularly in younger patients and in female patients in whom thoracic CT might raise the lifetime risk of breast cancer [324]. V/Q lung scintigraphy is diagnostic (with either normal- or high-probability findings) in $\sim 30-50 \%$ of emergency ward patients with suspected $\mathrm{PE}[75,122,134,325]$. The proportion of diagnostic V/Q scans is higher in patients with a normal chest X-ray, and this might support the use of a V/Q scan as a first-line imaging test for PE in younger patients, depending on local availability [326]. The number of patients with inconclusive findings may further be reduced by taking into account clinical probability. Thus, patients with a non-diagnostic lung scan and low clinical probability of PE have a low prevalence of confirmed PE $[124,325]$, and the negative predictive value of this combination is further increased by the absence of a DVT on lower-limb CUS. If a high-probability lung scan is obtained from a patient with low clinical probability of $\mathrm{PE}$, confirmation by other tests should be considered.

\subsection{Treatment strategies}

\subsubsection{Emergency treatment of high-risk pulmonary embolism}

The algorithm for a risk-adjusted therapeutic approach to acute PE is shown in figure 6; an emergency management algorithm specifically for patients with suspected acute high-risk PE is proposed in supplementary figure 1. Primary reperfusion treatment, in most cases systemic thrombolysis, is the treatment of choice for patients with high-risk PE. Surgical pulmonary embolectomy or percutaneous catheter-directed treatment are alternative reperfusion options in patients with contraindications to thrombolysis, if expertise with either of these methods and the appropriate resources are available on-site.

Following reperfusion treatment and haemodynamic stabilization, patients recovering from high-risk PE can be switched from parenteral to oral anticoagulation. As patients belonging to this risk category were excluded from the phase III NOAC trials, the optimal time point for this transition has not been determined by existing evidence but should instead be based on clinical judgement. The specifications concerning the higher initial dose of apixaban or rivaroxaban (for 1 and 3 weeks after PE diagnosis, respectively), or the minimum overall period (5 days) of heparin anticoagulation before switching to dabigatran or edoxaban, must be followed (see supplementary table 8 for tested and approved regimens). 


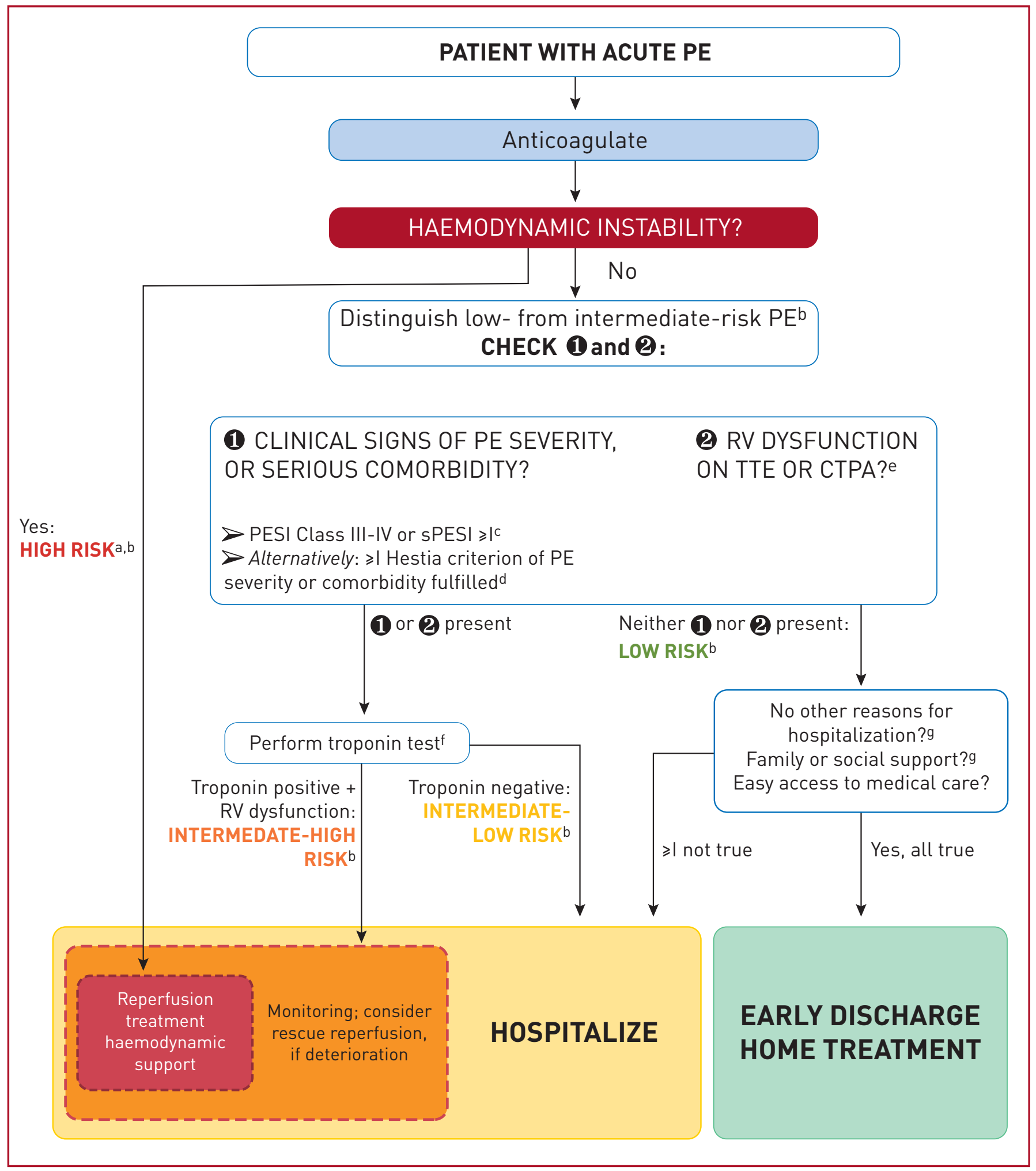

FIGURE 6 Central Illustration. Risk-adjusted management strategy for acute pulmonary embolism. CTPA: computed tomography pulmonary angiography/angiogram; PE: pulmonary embolism; PESI: Pulmonary Embolism Severity Index; RV: right ventricular; sPESI: simplified Pulmonary Embolism Severity Index; TTE: transthoracic echocardiogram. ${ }^{a}$ See also emergency management algorithm shown in the supplementary data. ${ }^{\mathrm{b}}$ Refer to table 8 for definition of high, intermediate-high-, intermediate-low-, and low-risk PE. 'Cancer, heart failure and chronic lung disease

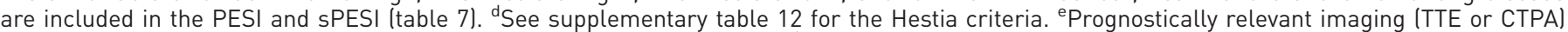
findings in patients with acute PE, are graphically presented in Figure $3 .{ }^{\mathrm{f}} \mathrm{A}$ cardiac troponin test may already have been performed during initial diagnostic work-up. ${ }^{9}$ Included in the Hestia criteria. 


\subsubsection{Treatment of intermediate-risk pulmonary embolism}

For most cases of acute PE without haemodynamic compromise, parenteral or oral anticoagulation (without reperfusion techniques) is adequate treatment. As shown in figure 6, normotensive patients with at least one indicator of elevated PE-related risk, or with aggravating conditions or comorbidity, should be hospitalized. In this group, patients with signs of RV dysfunction on echocardiography or CTPA (graphically presented in figure 3), accompanied by a positive troponin test, should be monitored over the first hours or days due to the risk of early haemodynamic decompensation and circulatory collapse [179]. Routine primary reperfusion treatment, notably full-dose systemic thrombolysis, is not recommended, as the risk of potentially life-threatening bleeding complications appears too high for the expected benefits from this treatment [179]. Rescue thrombolytic therapy or, alternatively, surgical embolectomy or percutaneous catheter-directed treatment should be reserved for patients who develop signs of haemodynamic instability. In the PEITHO trial, the mean time between randomization and death or haemodynamic decompensation was $1.79 \pm 1.60$ days in the placebo (heparin-only) arm [179]. Therefore, it appears reasonable to leave patients with intermediate-high-risk PE on LMWH anticoagulation over the first 2-3 days and ensure that they remain stable before switching to oral anticoagulation. As mentioned in the previous section, the specifications concerning the increased initial dose of apixaban or rivaroxaban, or the minimum overall period of heparin anticoagulation before switching to dabigatran or edoxaban, must be followed.

Suggestions for the anticoagulation and overall management of acute PE in specific clinical situations, for which conclusive evidence is lacking, are presented in supplementary table 9.

7.2.3. Management of low-risk pulmonary embolism: triage for early discharge and home treatment As a general rule, early discharge of a patient with acute PE and continuation of anticoagulant treatment at home should be considered if three sets of criteria are fulfilled: (i) the risk of early PE-related death or serious complications is low (section 5); (ii) there is no serious comorbidity or aggravating condition(s) (section 5) that would mandate hospitalization; and (iii) proper outpatient care and anticoagulant treatment can be provided, considering the patient's (anticipated) compliance, and the possibilities offered by the healthcare system and social infrastructure.

Randomized trials and prospective management cohort studies that investigated the feasibility and safety of early discharge, and home treatment, of PE adhered to these principles, even though slightly different criteria or combinations thereof were used to ensure the above three requirements.

The Hestia exclusion criteria (supplementary table 12) represent a checklist of clinical parameters or questions that can be obtained/answered at the bedside. They integrate aspects of PE severity, comorbidity, and the feasibility of home treatment. If the answer to one or more of the questions is "yes", then the patient cannot be discharged early. In a single-arm management trial that used these criteria to select candidates for home treatment, the 3 month rate of recurrent VTE was $2.0 \%(0.8-4.3 \%)$ in patients with acute PE who were discharged within $24 \mathrm{~h}$ [317]. In a subsequent non-inferiority trial that randomized 550 patients to direct discharge based on the Hestia criteria alone versus additional NT-proBNP testing and discharge if levels were $\leqslant 500 \mathrm{pg} / \mathrm{mL}$, the primary outcome (30 day PE- or bleeding-related mortality, cardiopulmonary resuscitation, or admission to an intensive care unit) was very low in both arms. The results suggest no incremental value of natriuretic-peptide testing in patients who are eligible for home treatment based on the Hestia criteria, although the study was not powered to exclude this possibility [318].

The PESI and its simplified form, the sPESI (table 7), also integrate clinical parameters of PE severity and comorbidity to permit assessment of overall 30 day mortality. Compared with the Hestia criteria, the PESI is more standardized, but it contains a less-comprehensive list of aggravating conditions; moreover, the sPESI excludes all patients with cancer from the low-risk category (compare table 7 with supplementary table 12). The PESI was not primarily developed as a tool to select candidates for home treatment, but it has been used - in combination with additional feasibility criteria - in a trial of 344 patients randomized to inpatient versus outpatient treatment of PE [178]. One (0.6\%) patient in each treatment group died within 90 days [178].

In patients who were included in prospective cohort studies and treated at home, with or without a short hospitalization period, the 3 month rates of thromboembolic recurrence, major bleeding, and death were $1.75,1.43$, and $2.83 \%$, respectively [327].

In summary, the currently available evidence indicates that both the Hestia rule and the PESI or sPESI appear capable of reliably identifying patients who are (i) at low PE-related risk, and (ii) free of serious comorbidity. Consequently, either may be used for clinical triage according to local experience and preference. If a PESI- or sPESI-based approach is chosen, it must be combined with assessment of the feasibility of early discharge and home treatment; this assessment is already integrated into the Hestia criteria. 
A more difficult decision related to immediate or early discharge is whether the exclusion of intermediate-risk $\mathrm{PE}$ on clinical grounds alone is adequate, or whether the assessment of RV dysfunction or myocardial injury (see section 5) by an imaging test or a laboratory biomarker is necessary to provide maximal safety for the patient in this "vulnerable" early period. A systematic review and meta-analysis of cohort studies suggested that the prognostic sensitivity is increased further when clinical criteria (e.g. PESI or sPESI) are combined with imaging findings, or laboratory biomarker levels [234]. A multicentre prospective management trial tested this hypothesis, investigating the efficacy and safety of early discharge, and ambulatory rivaroxaban treatment, in patients selected by clinical criteria and an absence of RV dysfunction. Overall, $20 \%$ of the screened unselected patients with PE were included. At the predefined interim analysis of 525 patients $(50 \%$ of the planned population), the 3 month rate of symptomatic or fatal recurrent VTE was $0.6 \%$ (one-sided upper $99.6 \%$ CI 2.1\%), permitting the early rejection of the null hypothesis and termination of the trial. Major bleeding occurred in six $(1.2 \%)$ of the patients in the safety population. There were no PE-related deaths [319]. In view of the existing evidence-and taking into consideration (i) the catastrophic scenario of early death if a patient with acute PE is falsely judged to be at low risk on clinical grounds alone and discharged "too early" (as described in a prematurely terminated trial [328]), and (ii) the ease and minimal additional effort of assessing RV size and function at presentation by echocardiography, or on the CTPA performed to diagnose the PE event itself [329] (section 5) - it is wise to exclude RV dysfunction and right heart thrombi if immediate or early (within the first 24-48 h) discharge of the patient is planned.

\section{Chronic treatment and prevention of recurrence}

The aim of anticoagulation after acute PE is to complete the treatment of the acute episode and prevent recurrence of VTE over the long-term. Current drugs and regimens for the initial phase, and the first months of anticoagulant treatment, are described in section 6.

Most of the randomized studies focusing on long-term anticoagulation for VTE have included patients with DVT, with or without PE; only two randomized studies have specifically focused on patients with PE $[330,331]$. The incidence of recurrent VTE does not appear to depend on the clinical manifestation of the first event (i.e. it is similar after PE and after proximal DVT). However, in patients who have had a PE, VTE more frequently recurs as PE, while in patients who have had a DVT, it tends to recur more frequently as DVT [332]. As a consequence, the case fatality rate of recurrent VTE in patients who have previously had a PE is twice as high as that of VTE recurrence after DVT $[333,334]$.

Landmark clinical trials have evaluated various durations of anticoagulant treatment with VKAs for VTE [330, 331, 335-337]. The findings of these studies permit the following conclusions. First, all patients with PE should receive $\geqslant 3$ months of anticoagulant treatment. Second, after withdrawal of anticoagulant treatment, the risk of recurrence is expected to be similar if anticoagulants are stopped after 3-6 months compared with longer treatment periods (e.g. 12-24 months). Third, extended oral anticoagulant treatment reduces the risk for recurrent VTE by $\leqslant 90 \%$, but this benefit is partially offset by the risk of bleeding.

Oral anticoagulants are highly effective in preventing recurrent VTE during treatment, but they do not eliminate the risk of subsequent recurrence after the discontinuation of treatment [330, 331]. Based on this fact on the one hand, and considering the bleeding risk of anticoagulation treatment on the other, the clinically important question is how to best select candidates for extended or indefinite anticoagulation. Involvement of the patient in the decision-making process is crucial to optimize and maintain treatment adherence.

\subsection{Assessment of venous thromboembolism recurrence risk}

The risk for recurrent VTE after discontinuation of treatment is related to the features of the index PE (or, in the broader sense, VTE) event. A study, which followed patients after a first episode of acute PE, found that the recurrence rate after discontinuation of treatment was $\sim 2.5 \%$ per year after PE associated with transient risk factors, compared with $4.5 \%$ per year after PE occurring in the absence of known cancer, known thrombophilia, or any transient risk factor [331]. Similar observations were made in other prospective studies in patients with DVT [337]. Advancing the concept further, randomized anticoagulation trials over the past 15 years, which have focused on secondary VTE prevention, have classified patients into distinct groups based on their risk of VTE recurrence after discontinuation of anticoagulant treatment. In general, these groups are: (i) patients in whom a strong (major) transient or reversible risk factor, most commonly major surgery or trauma, can be identified as being responsible for the acute (index) episode; (ii) patients in whom the index episode might be partly explained by the presence of a weak (minor) transient or reversible risk factor, or if a non-malignant risk factor for thrombosis persists; (iii) patients in whom the index episode occurred in the absence of any identifiable risk factor (the present Guidelines avoid terms such as "unprovoked" or "idiopathic" VTE); (iv) patients with one or more previous episodes of VTE, and those with a major persistent pro-thrombotic condition such as antiphospholipid antibody syndrome; and (v) patients with active cancer [338]. 
Table 11 shows examples of transient/reversible and persistent risk factors for VTE, classified by the risk of long-term recurrence. As active cancer is a major risk factor for recurrence of VTE, but also for bleeding while on anticoagulant treatment [339], section 8.4 is specifically dedicated to the management of $\mathrm{PE}$ in patients with cancer.

Overall, assessment of the VTE recurrence risk after acute PE, in the absence of a major transient or reversible risk factor, is a complex issue. Beyond the examples listed in table 11, patients who are carriers of some forms of hereditary thrombophilia, notably those with confirmed deficiency of antithrombin, protein $\mathrm{C}$, or protein $\mathrm{S}$, and patients with homozygous factor $\mathrm{V}$ Leiden or homozygous prothrombin G20210A mutation, are often candidates for indefinite anticoagulant treatment after a first episode of PE occurring in the absence of a major reversible risk factor. In view of these possible implications, testing for thrombophilia (including antiphospholipid antibodies and lupus anticoagulant) [342] may be considered in patients in whom VTE occurs at a young age (e.g. aged $<50$ years) and in the absence of an otherwise identifiable risk factor, especially when this occurs against the background of a strong family history of VTE. In such cases, testing may help to tailor the regimen and dose of the anticoagulant agent over the long-term. On the other hand, no evidence of a clinical benefit of extended anticoagulant treatment is currently available for carriers of heterozygous factor V Leiden or prothrombin 20210A mutation.

A number of risk prediction models have been developed for the assessment of the risk of recurrence in an individual patient (supplementary table 13) $[343,344]$. The clinical value and, in particular, the possible therapeutic implications of these models in the NOAC era are unclear.

\subsection{Anticoagulant-related bleeding risk}

Incidence estimates from cohort studies conducted more than 15 years ago reported an $\sim 3 \%$ annual incidence of major bleeding in patients treated with VKAs [345]. Meta-analyses of phase III studies

TABLE 11 Categorization of risk factors for venous thromboembolism based on the risk of recurrence over the long-term

\begin{tabular}{|c|c|c|}
\hline Estimated risk for long-term recurrence ${ }^{a}$ & Risk factor category for index PEb & Examplesb \\
\hline Low (<3\% per year) & $\begin{array}{l}\text { Major transient or reversible factors associated } \\
\text { with }>10 \text {-fold increased risk for the index VTE } \\
\text { event (compared to patients without the risk } \\
\text { factor) }\end{array}$ & $\begin{array}{l}\text { - Surgery with general anaesthesia for } \\
>30 \text { min } \\
\text { - Confined to bed in hospital (only "bathroom } \\
\text { privileges") for } \geqslant 3 \text { days due to an acute } \\
\text { illness, or acute exacerbation of a chronic } \\
\text { illness } \\
\text { - Trauma with fractures }\end{array}$ \\
\hline \multirow[t]{3}{*}{ Intermediate (3-8\% per year) } & $\begin{array}{l}\text { Transient or reversible factors associated with } \\
\leqslant 10 \text {-fold increased risk for first (index) VTE }\end{array}$ & $\begin{array}{l}\text { - Minor surgery (general anaesthesia for } \\
<30 \text { min) } \\
\text { - Admission to hospital for }<3 \text { days with an } \\
\text { acute illness } \\
\text { - Oestrogen therapy/contraception } \\
\text { - Pregnancy or puerpium } \\
\text { - Confined to bed out of hospital for } \geqslant 3 \text { days } \\
\text { with an acute illness } \\
\text { - Leg injury (without fracture) associated with } \\
\text { reduced mobility } \geqslant 3 \text { days } \\
\text { - Long-haul flight }\end{array}$ \\
\hline & Non-malignant persistent risk factors & $\begin{array}{l}\text { - Inflammatory bowel disease } \\
\text { - Active autoimmune disease }\end{array}$ \\
\hline & No identifiable risk factor & \\
\hline High (>8\% per year) & & $\begin{array}{l}\text { - Active cancer } \\
\text { - One or more previous episodes of VTE in the } \\
\text { absence of a major transient or reversible } \\
\text { factor } \\
\text { - Antiphospholipid antibody syndrome }\end{array}$ \\
\hline
\end{tabular}

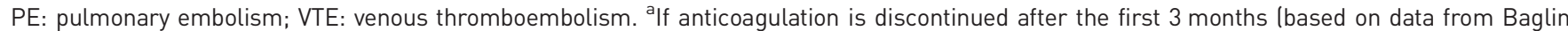
et al. [340] and lorio et al. [341]). ${ }^{\text {b}}$ The categorization of risk factors for the index VTE event is in line with that proposed by the International Society on Thrombosis and Haemostasis [338]. The present Guidelines avoid terms such as "provoked", "unprovoked", or "idiopathic" VTE. 
focusing on the first 3-12 months of anticoagulant treatment showed an $\sim 40 \%$ reduction in the risk for major bleeding with NOACs compared with VKAs [346]. The risk of major bleeding is higher in the first month of anticoagulant treatment, and then declines and remains stable over time. Based on currently available evidence, risk factors include: (i) advanced age (particularly $>75$ years); (ii) previous bleeding (if not associated with a reversible or treatable cause) or anaemia; (iii) active cancer; (iv) previous stroke, either haemorrhagic or ischaemic; (v) chronic renal or hepatic disease; (vi) concomitant antiplatelet therapy or non-steroidal anti-inflammatory drugs (to be avoided, if possible); (vii) other serious acute or chronic illness; and (viii) poor anticoagulation control.

Existing bleeding risk scores and their current validation status are reviewed in supplementary table 14 . The patient's bleeding risk should be assessed, either by implicit judgement after evaluating individual risk factors or by the use of a bleeding risk score, at the time of initiation of anticoagulant treatment. It should be reassessed periodically (e.g. once a year in patients at low risk, and every 3 or 6 months in patients at high risk for bleeding). Bleeding risk assessment should be used to identify and treat modifiable bleeding risk factors, and it may influence decision-making on the duration and regimen/dose of anticoagulant treatment after acute PE.

\subsection{Regimens and treatment durations with non-vitamin $K$ antagonist oral anticoagulants, and with other non-vitamin $K$ antagonist antithrombotic drugs}

All patients with PE should be treated with anticoagulants for $\geqslant 3$ months [347]. Beyond this period, the balance between the risk of VTE recurrence and that of bleeding, which has been used to select candidates for extended anticoagulation after a first VTE event in the VKA era, is currently being revisited based on the lower bleeding rates with NOACs. However, despite the improved safety of these drugs compared with VKAs, treatment with NOACs is not without risk. Phase III clinical trials on the extended treatment of VTE have shown that the rate of major bleeding may be $\sim 1 \%$, and that of clinically relevant non-major (CRNM) bleeding as high as $6 \%$. Bleeding rates may be higher in everyday clinical practice $[348,349]$.

The NOAC trials that focused on extended VTE treatment are summarized in supplementary table 15 . In all studies, patients with PE made up approximately one-third of the entire study population, while the remaining two-thirds were patients with proximal DVT but no clinically overt PE. Patients needed to have completed the initial and long-term anticoagulation phase to be included in the extended studies.

Dabigatran was compared with warfarin or placebo in two different studies (supplementary table 15) [350]. In these studies, dabigatran was non-inferior to warfarin for the prevention of confirmed recurrent symptomatic VTE or VTE-related death, and more effective than placebo for the prevention of symptomatic recurrent VTE or unexplained death [350]. The rate of major bleeding was $0.9 \%$ with dabigatran compared to $1.8 \%$ with warfarin (HR 0.52, 95\% CI 0.27-1.02) [350].

Rivaroxaban was compared with placebo or aspirin in two different studies in patients who had completed 6-12 months of anticoagulation treatment for a first VTE event (supplementary table 15). Treatment with rivaroxaban $[20 \mathrm{mg}$ once a day (o.d.)] reduced recurrent VTE by $\sim 80 \%$, with a $6.0 \%$ incidence of major or CRNM bleeding as compared to $1.2 \%$ with placebo [351]. Rivaroxaban given at a dose of 20 or $10 \mathrm{mg}$ o.d. was compared with aspirin (100 mg o.d.) in 3365 patients [352]. Both doses of rivaroxaban reduced symptomatic recurrent fatal or non-fatal VTE by $\sim 70 \%$ in comparison with aspirin. No significant differences in the rates of major or CRNM bleeding were shown between either dose of rivaroxaban and aspirin [352].

Patients with VTE were randomized to receive two different doses of apixaban [2.5 or $5 \mathrm{mg}$ twice a day (bis in die: b.i.d.)] or placebo after 6-12 months of initial anticoagulation (supplementary table 15) [353]. Both doses of apixaban reduced the incidence of symptomatic recurrent VTE or death from any cause compared with placebo, with no safety concerns [353].

Patients at high bleeding risk — based on the investigator's judgement, the patient's medical history, and the results of laboratory examinations-were excluded from the extension studies mentioned above; this was also the case for studies on extended anticoagulation with VKAs [330, 331]. This fact should be taken into account during triage of a patient for extended anticoagulation with one of the above regimens.

In a randomized, open-label study in high-risk patients with antiphospholipid syndrome (testing triple positive for lupus anticoagulant, anticardiolipin, and anti- $\beta 2$-glycoprotein I), rivaroxaban was associated with an increased rate of thromboembolic and major bleeding events compared with warfarin (HR for the composite primary outcome 6.7; 95\% CI 1.5-30.5) [354]. At present, NOACs are not an alternative to VKAs for patients with antiphospholipid syndrome.

In two trials with a total of 1224 patients, extended therapy with aspirin (after termination of standard oral anticoagulation) was associated with a $30-35 \%$ reduction in the risk of recurrence compared with 
placebo (supplementary table 15) [355, 356]. However, more recently, another trial demonstrated the superiority of anticoagulation with rivaroxaban, either 20 or $10 \mathrm{mg}$ o.d., over aspirin for secondary prophylaxis of VTE recurrence [352].

A randomized, placebo controlled study evaluated sulodexide $(2 \times 250$ lipasemic unit capsules b.i.d.) for the prevention of recurrence in 615 patients with a first VTE event without an identifiable risk factor, who had completed 3-12 months of oral anticoagulant treatment (supplementary table 15) [357]. Sulodexide reduced the risk of recurrence by $\sim 50 \%$ with no apparent increase in bleeding events. However, only $8 \%$ of patients in this study had PE as the index VTE event [357].

\subsection{Recommendations for the regimen and duration of anticoagulation after pulmonary embolism in patients without cancer}

\section{Recommendations}

Class $^{a}$ Level $^{b}$

Therapeutic anticoagulation for $\geqslant 3$ months is recommended for all patients with PE [347].

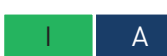

\section{Patients in whom discontinuation of anticoagulation after 3 months is recommended}

For patients with first PE/VTE secondary to a major transient/reversible risk factor, discontinuation of therapeutic oral anticoagulation is recommended after 3 months [331, $340,341]$.

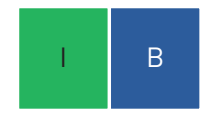

\section{Patients in whom extension of anticoagulation beyond 3 months is recommended}

Oral anticoagulant treatment of indefinite duration is recommended for patients presenting with recurrent VTE (that is, with at least one previous episode of PE or DVT) not related to a major transient or reversible risk factor [358].

Oral anticoagulant treatment with a VKA for an indefinite period is recommended for patients with antiphospholipid antibody syndrome [359].

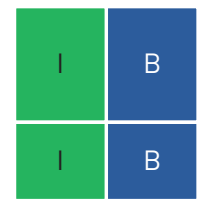

Patients in whom extension of anticoagulation beyond 3 months should be consideredc,d

Extended oral anticoagulation of indefinite duration should be considered for patients with a first episode of PE and no identifiable risk factor [330, 331, 347, 351-353].

Extended oral anticoagulation of indefinite duration should be considered for patients with a first episode of PE associated with a persistent risk factor other than antiphospholipid antibody syndrome $[330,352,353]$.

Extended oral anticoagulation of indefinite duration should be considered for patients with a first episode of PE associated with a minor transient or reversible risk factor [330, 331, 352].

\section{NOAC dose in extended anticoagulatione}

If extended oral anticoagulation is decided after $\mathrm{PE}$ in a patient without cancer, a reduced dose of the NOACs apixaban (2.5 mg b.i.d.) or rivaroxaban (10 mg o.d.) should be considered after 6 months of therapeutic anticoagulation $[352,353]$.

\section{Extended treatment with alternative antithrombotic agents}

In patients who refuse to take or are unable to tolerate any form of oral anticoagulants, aspirin or sulodexide may be considered for extended VTE prophylaxis [355-357].

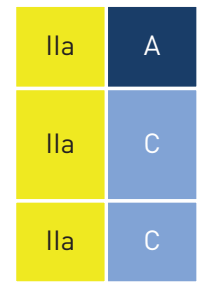

\section{Follow-up of the patient under anticoagulation}

In patients who receive extended anticoagulation, it is recommended that their drug tolerance and adherence, hepatic and renalf function, and bleeding risk be reassessed at regular intervals [259].

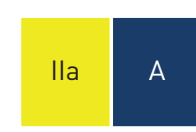

b.i.d.: bis in die (twice a day); DVT: deep vein thrombosis; NOAC(s): non-vitamin $\mathrm{K}$ antagonist oral anticoagulant(s); o.d.: omni die (once a day); PE: pulmonary embolism; VKA: vitamin K antagonist; VTE: venous thromboembolism. ${ }^{\mathrm{a}}$ Class of recommendation. ${ }^{\mathrm{b}}$ Level of evidence. ${ }^{\mathrm{C}}$ The patient's bleeding risk should be assessed (see supplementary table 14 for prediction models) to identify and treat modifiable bleeding risk factors, and it may influence decision-making on the duration and regimen/dose of anticoagulant treatment. ${ }^{d}$ Refer to supplementary table 9 for therapeutic decisions in specific clinical situations. ${ }^{\text {If }}$ dabigatran or edoxaban is chosen for extended anticoagulation after PE, the dose should remain unchanged, as reduced-dose regimens were not investigated in dedicated extension trials [313, 350]. 'Especially for patients receiving NOACs.

\subsection{Management of pulmonary embolism in patients with cancer}

Five RCTs compared LMWH versus conventional VTE treatment (heparin followed by VKA) in the treatment of VTE in cancer-associated thrombosis [360-364]. In 2003, a significant reduction in VTE 
recurrence was reported with LMWH compared with conventional (VKA) treatment without an increase in bleeding complications [362]. In a more recent trial, long-term administration of tinzaparin failed to achieve a statistically significant reduction in overall VTE recurrence over conventional treatment (HR 0.65, 95\% CI 0.41-1.03); however, the overall rate of recurrent VTE in the control arm was lower than that previously observed, probably as a result of the recruitment of patients with a lower cancer-specific thrombotic risk [360]. Overall, LMWHs were found to decrease the risk of recurrent VTE by $40 \%$ with a risk of major bleeding complications similar to that of VKAs [365]. Accordingly, LMWHs have become the standard of care. However, these agents are associated with a relevant cost and burden for patients. In addition, the absolute rate of recurrent VTE while on LMWH remains high (7-9\%) compared with that observed in non-cancer patients with VTE on conventional treatment (1.5-3\%) [365].

NOACs could make the treatment of VTE easier and more convenient in patients with cancer, due to their oral administration in fixed-dose regimens and their lower cost compared with LMWH. However, only 3-9\% of patients included in phase III studies with NOACs for the treatment of VTE had concomitant cancer [260, 261, 312, 314, 351]. A randomized, open-label trial compared edoxaban with LMWH in the secondary prevention of VTE in 1050 patients with cancer-associated thrombosis (mostly symptomatic or asymptomatic PE) [366]. Edoxaban (60 mg o.d., reduced to $30 \mathrm{mg}$ in subjects with moderate renal impairment, low body weight, or concomitant need for strong inhibitors of glycoprotein-P) was started after 5 days of LMWH and treatment was given for $\geqslant 6$ months. Edoxaban was non-inferior to dalteparin in the prevention of VTE recurrence or major bleeding over 12 months after randomization (HR 0.97, $95 \%$ CI $0.70-1.36$ ). Major bleeding occurred in $6.9 \%$ of the patients in the edoxaban arm and $4.0 \%$ in the dalteparin arm (difference in risk 2.9 percentage points, 95\% CI 0.1-5.6). This difference appears to have been mainly accounted for by the high rate of bleeding in patients with gastrointestinal cancer allocated to the edoxaban group [366]. Similar results were reported by a randomized, open-label pilot trial comparing rivaroxaban with dalteparin in 406 patients with VTE and cancer, 58\% of whom had metastases [367]. A significant decrease in the risk of recurrent VTE was observed with rivaroxaban (HR $0.43,95 \% \mathrm{CI}$ 0.19-0.99). The 6 month cumulative rate of major bleeding, which was mostly gastrointestinal, was $6 \%$ (95\% CI 3-11\%) for rivaroxaban and 4\% (95\% CI 2-8\%) for dalteparin (HR 1.83, 95\% CI 0.68-4.96). Corresponding rates of CRNM bleeds were $13 \%$ (95\% CI 9-19\%) and 4\% (95\% CI 2-9\%), respectively (HR 3.76, 95\% CI 1.63-8.69) [367].

Based on the currently available evidence, as described above, patients with acute PE and cancer, particularly those with gastrointestinal cancer, should be encouraged to continue LMWH for $\geqslant 3-6$ months. This also applies to patients in whom oral treatment is unfeasible due to problems of intake or absorption, and to those with severe renal impairment. In all other cases, especially in patients with an anticipated low risk of bleeding and without gastrointestinal tumours, the choice between LMWH and edoxaban or rivaroxaban is left to the discretion of the physician, and the patient's preference.

Owing to the high risk for recurrence, patients with cancer should receive indefinite anticoagulation after a first episode of VTE. Although existing evidence is limited, it is conceivable that once cancer is cured the risk for recurrence decreases and anticoagulation can be stopped. However, the definition of cured cancer is not always clear. The risk of recurrence of $\mathrm{PE}$ in cancer was assessed in a cohort study of 543 patients and was validated in an independent set of 819 patients [368]. The proposed score to predict the risk of recurrence included breast cancer (minus 1 point), Tumour Node Metastasis stage I or II (minus 1 point), and female sex, lung cancer, and previous VTE (plus 1 point each). Patients with a score $\leqslant 0$ were at low risk $(\leqslant 4.5 \%)$ and those with a score $\geqslant 1$ were at high $(\geqslant 19 \%)$ risk of VTE recurrence over the first 6 months [368].

After the first 3-6 months, extended anticoagulation may consist of continuation of LMWH or transition to an oral anticoagulant. Two cohort studies have assessed the safety of extended treatment with LMWH ( $\leqslant 12$ months) in cancer-associated thrombosis $[369,370]$. In both studies, the incidence of bleeding complications was higher in the first months and then reached a plateau that remained unchanged after the sixth month. In the absence of conclusive evidence, the decision to continue with LMWH or to change to VKA or a NOAC should be made on a case-by-case basis after consideration of the success of anticancer therapy, the estimated risk of recurrence of VTE, the bleeding risk, and the preference of the patient. Periodic reassessment of the risk-to-benefit ratio of continued anticoagulant treatment is mandatory.

As mentioned in section 5, venous filters are principally indicated when anticoagulation is impossible due to active haemorrhage or an excessive bleeding risk. However, the risk of VTE recurrence in the absence of anticoagulation is particularly high in patients with cancer, and the insertion of a filter should not delay the initiation of anticoagulation as soon as it is safe to do so. There is no evidence to support the use of venous filters as an adjunct to anticoagulation treatment in patients with cancer. 
A number of studies have reported that a proportion of patients presenting with PE in the absence of identifiable risk factors develop cancer within the first year after diagnosis [371]. Consequently, the optimal strategy to achieve early diagnosis of these occult cancers was investigated. Two large randomized trials failed to show that comprehensive CT of the abdomen or ${ }^{18} \mathrm{~F}$ deoxy-fluoro-glucose positron emission tomography were able to detect more cancers than limited screening in patients with an unprovoked VTE $[372,373]$. Therefore, based on current evidence, the search for occult cancer after an episode of VTE may be restricted to careful history taking, physical examination, basic laboratory tests, and a chest X-ray (if no CTPA was performed to diagnose PE) [372, 374, 375].

In patients with cancer, incidental PE should be managed in the same manner as symptomatic PE, whether it involves segmental or more proximal branches, multiple subsegmental vessels, or a single subsegmental vessel in association with detectable DVT [376, 377].

\subsection{Recommendations for the regimen and the duration of anticoagulation after pulmonary embolism in patients with active cancer}

\section{Recommendations}

For patients with PE and cancer, weight-adjusted subcutaneous LMWH should be considered for the first 6 months over VKAs [360-363].

Edoxaban should be considered as an alternative to weight-adjusted subcutaneous LMWH in patients without gastrointestinal cancer [366].

Rivaroxaban should be considered as an alternative to weight-adjusted subcutaneous LMWH in patients without gastrointestinal cancer [367].

For patients with PE and cancer, extended anticoagulation (beyond the first 6 months)c should be considered for an indefinite period or until the cancer is cured [378].

In patients with cancer, management of incidental PE in the same manner as symptomatic PE should be considered, if it involves segmental or more proximal branches, multiple subsegmental vessels, or a single subsegmental vessel in association with proven DVT [376, 377].

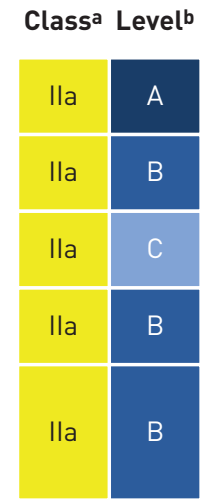

DVT: deep vein thrombosis; LMWH: low-molecular weight heparin; PE: pulmonary embolism; VKAs: vitamin $\mathrm{K}$ antagonists. ${ }^{\mathrm{a}} \mathrm{Class}$ of recommendation. ${ }^{\mathrm{b}}$ Level of evidence. ${ }^{\mathrm{C}}$ Refer to supplementary table 9 for further guidance on therapeutic decisions after the first 6 months.

\section{Pulmonary embolism and pregnancy}

\subsection{Epidemiology and risk factors for pulmonary embolism in pregnancy}

Acute PE remains one of the leading causes of maternal death in high-income countries [379, 380]. For example, in the UK and Ireland, thrombosis and thromboembolism were the most common causes of direct maternal death (death resulting from the pregnancy rather than pre-existing conditions) in the triennium 2013-15, resulting in 1.13 mortalities per 100000 maternities (www.npeu.ox.ac.uk/mbrrace-uk). VTE risk is higher in pregnant women compared with non-pregnant women of similar age; it increases during pregnancy and reaches a peak during the post-partum period [381]. The baseline pregnancy-related risk increases further in the presence of additional VTE risk factors, including in vitro fertilization: in a cross-sectional study derived from a Swedish registry, the HR for VTE following in vitro fertilization was 1.77 (95\% CI 1.41-2.23) overall and 4.22 (95\% CI 2.46-7.20) during the first trimester [382]. Other important and common risk factors include prior VTE, obesity, medical comorbidities, stillbirth, pre-eclampsia, post-partum haemorrhage, and caesarean section; documented risk assessment is therefore essential [383].

The recommendations provided in these Guidelines are in line with those included in the 2018 ESC Guidelines on the management of cardiovascular diseases during pregnancy [384].

\subsection{Diagnosis of pulmonary embolism in pregnancy \\ 9.2.1. Clinical prediction rules and D-dimers}

Diagnosis of PE during pregnancy can be challenging as symptoms frequently overlap with those of normal pregnancy. The overall prevalence of confirmed PE is low among women investigated for the disease, between 2 and 7\% [385-388]. D-dimer levels continuously increase during pregnancy [389, 390], and levels are above the threshold for VTE "rule-out" in almost one-quarter of pregnant women in the third trimester [390]. The results of a multinational prospective management study of 441 pregnant women presenting to emergency departments with clinically suspected PE suggest that a diagnostic strategy-based on the assessment of clinical probability, D-dimer measurement, CUS, and CTPA-may safely exclude PE in pregnancy [388]. In that study, PE exclusion on the basis of a negative D-dimer result 


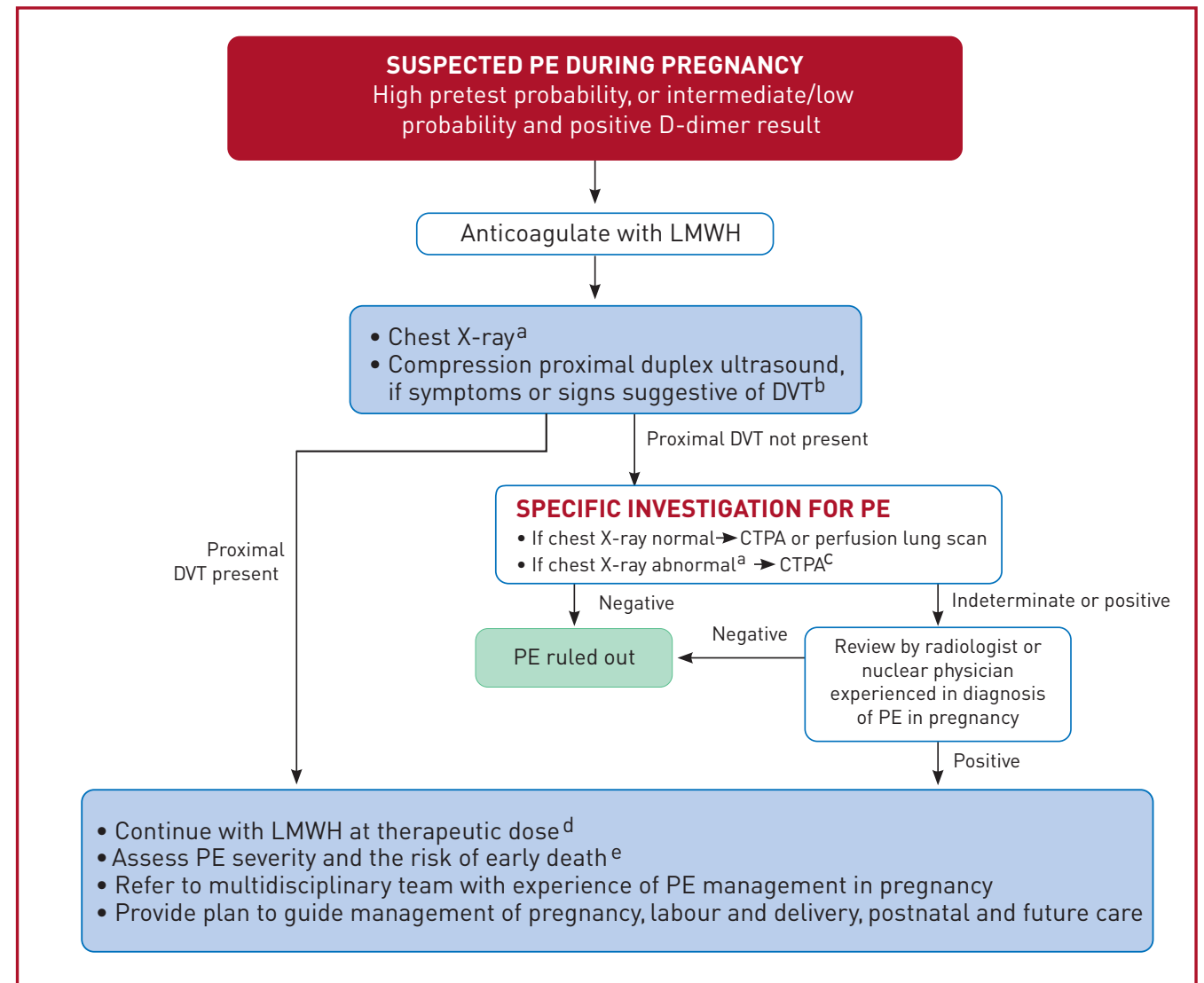

FIGURE 7 Diagnostic workup and management of suspected pulmonary embolism during pregnancy, and up to 6 weeks post-partum. CTPA: computed tomography pulmonary angiography; CUS: compression ultrasonography; DVT: deep vein thrombosis; LMWH: low-molecular-weight heparin; PE: pulmonary embolism. alf chest X-ray abnormal, consider also alternative cause of chest symptoms. ${ }^{b}$ DVT in pelvic veins may not be ruled out by CUS. If the entire leg is swollen, or there is buttock pain or other symptoms suggestive of pelvic thrombosis, consider magnetic resonance venography to rule out DVT. ' $C T P A$ technique must ensure very low fetal radiation exposure (see table 12). ${ }^{\mathrm{d}}$ Perform full blood count (to measure haemoglobin and platelet count) and calculate creatinine clearance before administration. Assess bleeding risk and ensure absence of contra-indications. ${ }^{\text {e}}$ See table 8.

(without imaging) was possible in $11.7 \%$ of the 392 women with a non-high pre-test probability (Geneva) score, a rate that was reduced to $4.2 \%$ in the third trimester [388]. A further prospective management study evaluated a combination of a pregnancy-adapted YEARS algorithm with D-dimer levels in 498 women with suspected PE during pregnancy. PE was ruled out without CTPA in women deemed to be at low PE risk according to the combination of the algorithm and D-dimer results. At 3 months, only one woman with PE excluded on the basis of the algorithm developed a popliteal DVT $(0.21 \%$, 95\% CI 0.04-1.2) and no women developed PE [391].

\subsubsection{Imaging tests}

A proposed algorithm for the investigation of suspected PE in women who are pregnant, or $\leqslant 6$ weeks post-partum, is shown in figure 7. Both maternal and fetal radiation exposure are low using modern imaging techniques (table 12) [385, 392-398]. For V/Q scans and CTPA, fetal radiation doses are well below the threshold associated with fetal radiation complications (which is $50-100 \mathrm{mSv}$ ) [399, 400]. In the past, CTPA has been reported to cause high radiation exposure to the breast [395, 401]; however, CT technology has evolved, and several techniques can now reduce radiation exposure without compromising image quality. These include reducing the anatomical coverage of the scan [393], reducing the kilovoltage, using iterative reconstructive techniques, and reducing the contrast-monitoring component of the CTPA $[392,393]$. Modern CTPA imaging techniques may therefore expose the maternal breast to median doses as low as 3-4 mGy (table 12) [392]. The effect on maternal cancer risk with modern CTPA techniques is negligible (lifetime cancer risk is reportedly increased by a factor of 1.0003-1.0007); avoiding CTPA on the grounds of maternal cancer risk is therefore not justified [394]. 


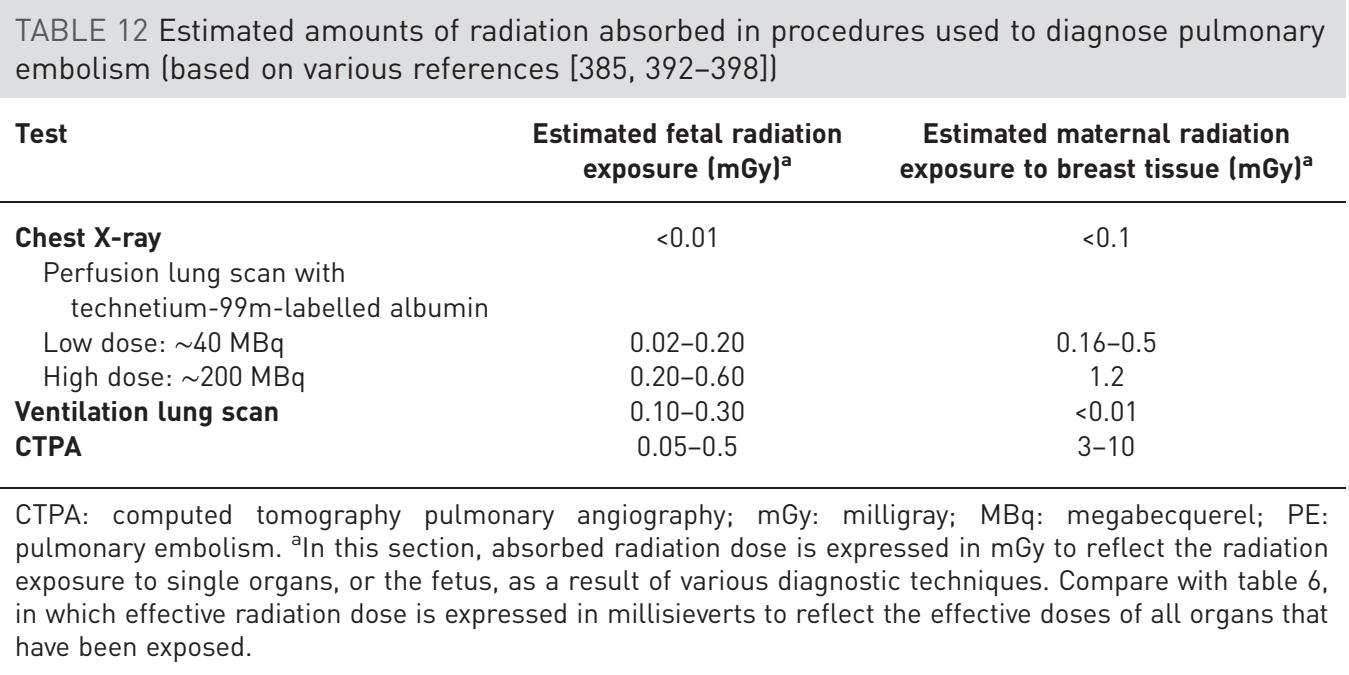

A normal perfusion scan and a negative CTPA appear equally safe for ruling out PE in pregnancy, as suggested by retrospective series [385, 386, 402-404]. Inconclusive results can be a problem $(4-33 \%$ of investigations) [385, 386, 405], especially late in pregnancy [405]. A recent survey of 24 sites in the UK, representing a population of 15.5 million, revealed a similar rate of inadequate or indeterminate CTPA and scintigraphy scans, suggesting that the initial choice of imaging is best determined by local expertise and resources [406].

V/Q SPECT is associated with low fetal and maternal radiation exposure, and has promise in PE diagnosis in pregnancy [407]. However, further evaluation of this technique is required before its widespread incorporation into diagnostic algorithms. For MRA, the long-term effects of gadolinium contrast on the fetus are not known. In non-pregnant patients, technically inadequate images are frequently obtained and the rate of inconclusive scan results is high [140]. Therefore, use of this technique for diagnosing or ruling out PE during pregnancy cannot be recommended at present. Conventional pulmonary angiography involves significantly higher radiation exposure of the fetus $(2.2-3.7 \mathrm{mSv})$ and should be avoided during pregnancy [400].

Overdiagnosis of PE is a potential pitfall that can have significant, lifelong implications for a pregnant woman, including the risk of bleeding at the time of delivery, the withholding of oestrogen contraception, and the requirement for thromboprophylaxis during future pregnancies. Consequently, avoiding PE overdiagnosis in pregnancy is as important as not missing a PE diagnosis.

\subsection{Treatment of pulmonary embolism in pregnancy}

LMWH is the treatment of choice for PE during pregnancy [384]. In contrast to VKAs and NOACs, LMWH does not cross the placenta, and consequently does not confer a risk of fetal haemorrhage or teratogenicity. Moreover, while UFH is also safe in pregnancy, LMWH has more predictable pharmacokinetics and a more favourable risk profile [408-411]. Although no RCT has evaluated the optimal dose of LMWH for the treatment of PE during pregnancy, currently published data favour similar dosing to non-pregnant patients, either with o.d. or b.i.d. regimens based on early pregnancy weight [408, 410]. For the majority of patients receiving LMWH treatment for PE during pregnancy [412, 413], it remains uncertain whether using serial measurements of plasma anti-activated coagulation factor $\mathrm{X}$ activity to guide dosing may be of clinical benefit. It is important to bear in mind that: (i) LMWH has a predictable pharmacokinetic profile, (ii) data on optimal anti-activated coagulation factor levels are lacking, and (iii) the assay itself has limitations [414]. In addition, there are no solid data on the clinical benefit versus harm of frequent, weight-based dose adjustments of LMWH during pregnancy. Thus, anti-activated coagulation factor $\mathrm{X}$ monitoring may be reserved for specific high-risk circumstances such as recurrent VTE, renal impairment, and extremes of body weight.

The use of UFH has been associated with heparin-induced thrombocytopenia and bone loss. It remains uncertain whether, and to what extent, the risk of bone loss is increased with LMWH use. In a recent observational cohort study, in which bone mineral density was measured by dual-energy X-ray absorptiometry 4-7 years after the last delivery in 152 women (92 of whom received prolonged LMWH during pregnancy), lumbar spine bone mineral density was similar in LWMH-treated women and 
controls following adjustment for potential confounders. No osteoporosis or osteoporotic fractures were reported [415].

Fondaparinux may be considered if there is an allergy or adverse response to LMWH, although solid data are lacking and minor transplacental passage has been demonstrated [416]. VKAs cross the placenta and are associated with a well-defined embryopathy during the first trimester. Administration of VKAs in the third trimester can result in fetal and neonatal haemorrhage, as well as placental abruption. Warfarin may be associated with central nervous system anomalies in the fetus throughout pregnancy. NOACs are contraindicated in pregnant patients [417].

The management of labour and delivery requires particular attention. In women receiving therapeutic LMWH, strong consideration should be given to planned delivery in collaboration with the multidisciplinary team to avoid the risk of spontaneous labour while fully anticoagulated. The incidence of spinal haematoma after regional anaesthesia is unknown in pregnant women under anticoagulation treatment. If regional analgesia is considered for a woman receiving therapeutic LMWH, $\geqslant 24 \mathrm{~h}$ should have elapsed since the last LMWH dose before insertion of a spinal or epidural needle (assuming normal renal function and including risk assessment at extremes of body weight).

In high-risk situations, for example in patients with recent $\mathrm{PE}$, it is recommended that LMWH be converted to UFH $\geqslant 36 \mathrm{~h}$ prior to delivery. The UFH infusion should be stopped $4-6 \mathrm{~h}$ prior to anticipated delivery and the activated partial thromboplastin time should be normal (i.e. not prolonged) prior to regional anaesthesia [418].

Data are limited on the optimal timing of post-partum reinitiation of LMWH [419, 420]. Timing will depend upon the mode of delivery and an assessment of the thrombotic versus bleeding risk by a multidisciplinary team. LMWH should not be given for $\geqslant 4 \mathrm{~h}$ after removal of the epidural catheter; the decision on timing and dose should consider whether the epidural insertion was traumatic, and take into account the risk profile of the woman. For example, an interim dose of a prophylactic LMWH dose may be considered post-operatively (after caesarean section), once at least $4 \mathrm{~h}$ have elapsed since epidural catheter removal, and allowing for an interval of $\geqslant 8-12 \mathrm{~h}$ between the prophylactic and the next therapeutic dose. Close collaboration between the obstetrician, the anaesthesiologist, and the attending physician is recommended.

Anticoagulant treatment should be administered for $\geqslant 6$ weeks after delivery and with a minimum overall treatment duration of 3 months. LMWH and warfarin can be given to breastfeeding mothers; the use of NOACs is not recommended [417].

High-risk, life-threatening $\mathrm{PE}$ during pregnancy is a rare, but potentially devastating, event. A recent systematic review included 127 cases of severe PE during pregnancy (and until 6 weeks post-partum) treated with thrombolysis, thrombectomy, and/or ECMO [421]. Both high- and intermediate-risk PE cases were included, and $23 \%$ of women experienced cardiac arrest. Reported survival rates were 94 and $86 \%$ following thrombolysis and surgical thrombectomy, respectively; however, these favourable rates may reflect reporting bias. Following thrombolysis, major bleeding occurred in 18 and 58\% of cases during pregnancy and in the post-partum period, respectively [421]. Finally, fetal deaths occurred in 12 and $20 \%$ of the cases following thrombolysis and thrombectomy, respectively [421]. Thrombolytic treatment should not be used peri-partum, except in the setting of life-threatening PE. Typically, UFH is used in the acute treatment of high-risk PE.

Although the indications for vena cava filters are similar to those for non-pregnant patients (discussed in section 6), there is limited experience with their use in pregnancy and the risk associated with the procedure may be increased.

Suggestions for the anticoagulation management of PE in specific clinical situations (also) related to pregnancy, for which conclusive evidence is lacking, are presented in supplementary table 9.

\subsubsection{Role of a multidisciplinary pregnancy heart team}

A team of multidisciplinary colleagues should collaborate in the planning of ante-, peri-, and post-partum care pathways for women with cardiovascular diseases, including PE. As many members as possible of this team should have expertise in the management of PE during pregnancy and the post-partum period. Jointly agreed, written care pathways should be available (if timelines permit) for effective communication (an example is shown in figure 7).

\subsection{Amniotic fluid embolism}

Amniotic fluid embolism (AFE) is a rare condition that occurs during pregnancy or shortly after delivery. It remains one of the leading causes of direct maternal death (i.e. death resulting from the pregnancy 
rather than from pre-existing conditions) in high-income countries [422]. Diagnosis of AFE is challenging, being primarily a clinical diagnosis of exclusion. Awareness of AFE, prompt diagnosis, and aggressive life support are of critical importance. AFE is characterized by unexplained sudden cardiovascular or respiratory deterioration, often accompanied by disseminated intravascular coagulation [422], and occurring during pregnancy or after delivery $[423,424]$. The reported incidence is approximately $2-7$ per 100000 maternities, with a mortality rate of 0.5-6 deaths per 100000 deliveries $[422,425,426]$. Reported case fatality rates vary, reflecting the challenges in making the diagnosis and the rarity of AFE. In a retrospective Californian study including more than 3.5 million deliveries, a case fatality rate of $13 \%$ was reported, as in other US and Canadian studies [425]. Similarly, a case fatality rate of 19\% was reported in a recent prospective UK population-based study with validated case criteria [422]. Recent literature have suggested that risk factors for AFE may include pre-existing cardiac, cerebrovascular, and renal disorders, placenta previa, polyhydramnios, stillbirth, chorioamnionitis, hypertensive disorders, instrumental delivery, and caesarean section [422, 425]. Management of AFE is supportive, and based on high-quality emergency care following the recognition and diagnosis of the condition, with prompt treatment of bleeding and coagulopathy [423]. Awareness of AFE should be integral to the education of involved physicians and to emergency algorithms.

\subsection{Recommendations for pulmonary embolism in pregnancy}

\section{Recommendations}

Class ${ }^{a}$ Level $^{b}$

\section{Diagnosis}

Formal diagnostic assessment with validated methods is recommended if PE is suspected during pregnancy or in the post-partum period [388, 391].

D-dimer measurement and clinical prediction rules should be considered to rule out PE during pregnancy or the post-partum period [388, 391]

In a pregnant patient with suspected PE (particularly if she has symptoms of DVT), venous CUS should be considered to avoid unnecessary irradiation [388].

Perfusion scintigraphy or CTPA (with a low-radiation dose protocol) should be considered to rule out suspected PE in pregnant women; CTPA should be considered as the first-line option if the chest X-ray is abnormal $[385,386]$.

\section{Treatment}

A therapeutic, fixed dose of LMWH based on early pregnancy body weight is the recommended therapy for $P E$ in the majority of pregnant women without haemodynamic instability $[408,410]$.

Thrombolysis or surgical embolectomy should be considered for pregnant women with high-risk PE [421].

Insertion of a spinal or epidural needle is not recommended, unless $\geqslant 24 \mathrm{~h}$ have passed since the last therapeutic dose of LMWH.

Administration of LMWH is not recommended within $4 \mathrm{~h}$ of removal of an epidural catheter NOACs are not recommended during pregnancy or lactation.

\section{Amniotic fluid embolism}

Amniotic fluid embolism should be considered in a pregnant or post-partum woman with otherwise unexplained cardiac arrest, sustained hypotension, or respiratory deterioration, especially if accompanied by disseminated intravascular coagulation $[422,425,426]$.
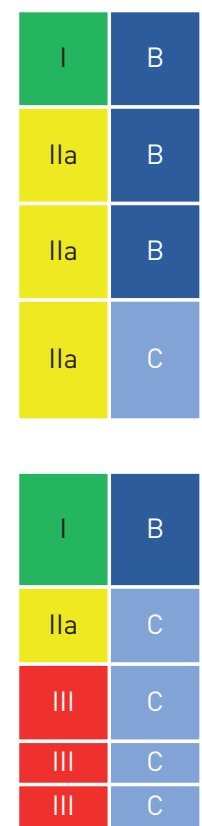

CTPA: computed tomography pulmonary angiography; CUS: compression ultrasonography; DVT: deep vein thrombosis; LMWH: low-molecular weight heparin; NOACs: non-vitamin K antagonist oral anticoagulants;

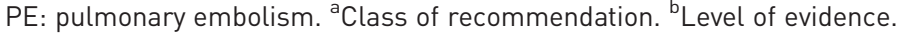

\section{Long-term sequelae of pulmonary embolism}

The patency of the pulmonary arterial bed is restored in the majority of PE survivors within the first few months following the acute episode; therefore, no routine follow-up CTPA imaging is needed in such patients treated for PE [427]. However, in other patients, thrombi become persistent and organized, which in rare cases may result in CTEPH, a potentially life-threatening obstructing vasculopathy. The rarity of this condition is in contrast to the relatively large number of patients who report persisting dyspnoea or poor physical performance over several months after acute PE. Thus, the aims of an efficient follow-up strategy after PE should be to: (i) provide appropriate care (exercise rehabilitation, treatment of 
comorbidity, behavioural education, and modification of risk factors) to patients with persisting symptoms, and (ii) ensure early detection of CTEPH to refer the patient for further diagnostic workup and specific treatment.

\subsection{Persisting symptoms and functional limitation after pulmonary embolism}

Cohort studies conducted over the past decade (summarized in Klok et al. [428]) have revealed that persisting or deteriorating dyspnoea, and poor physical performance, are frequently present 6 months to 3 years after an acute PE episode. The proportion of patients claiming that their health status is worse at 6 month follow-up than it was at the time of PE diagnosis varies widely, ranging between 20 and 75\% [429-431]. The following baseline parameters and findings could be identified as predictors of exertional dyspnoea at long-term follow-up after PE: advanced age, cardiac or pulmonary comorbidity, higher body mass index, and history of smoking [429]; higher systolic PAP and RV dysfunction at diagnosis [430, 432, 433]; and residual pulmonary vascular obstruction at discharge [434].

More recently, a prospective cohort study enrolled 100 patients at five Canadian hospitals between 2010 and 2013, and followed them over 1 year [435]. As many as $47 \%$ of the patients had reduced maximal aerobic capacity, defined as peak oxygen consumption $<80 \%$ of the predicted value on cardiopulmonary exercise testing (CPET). This functional outcome was associated with significantly worse generic health-related quality of life and dyspnoea scores, as well as with a significantly reduced 6 min walk distance [435]. Independent predictors of reduced functional exercise capacity and quality of life over time included female sex, higher body mass index, history of lung disease, higher pulmonary artery systolic pressures on the 10 day echocardiogram, and higher main pulmonary artery diameter on the baseline CTPA [436]. Of note, pulmonary function tests and echocardiographic results at follow-up were largely within normal limits, both in patients with and without reduced maximal aerobic capacity [435]. Lack of an association between exercise impairment, and persistent RV dilation or dysfunction, was also reported by a study of 20 survivors of massive or submassive PE [437].

Taken together, older and more recent cohort studies have suggested that muscle deconditioning, particularly in the presence of excess body weight and cardiopulmonary comorbidity, is largely responsible for the frequently reported dyspnoea and signs of exercise limitation after acute PE. This also means that, at least in the majority of cases, poor physical performance after PE does not appear to be attributable to "large" residual thrombi, or persisting/progressive $\mathrm{PH}$ and RV dysfunction. Ongoing prospective studies in large numbers of patients may help to better identify predictors of functional and/or haemodynamic impairment after acute $\mathrm{PE}$, and their possible implications for shaping follow-up programmes [438].

As mentioned in section 6, it remains unclear whether early reperfusion treatment, notably thrombolysis, has an impact on clinical symptoms, functional limitation, or persistent (or new-onset) PH at long-term follow-up after PE. Consequently, prevention of long-term PE sequelae is, at present, no justification for thrombolytic treatment in the acute phase of PE.

\subsection{Chronic thromboembolic pulmonary hypertension}

10.2.1. Epidemiology, pathophysiology, and natural history

CTEPH is a disease caused by the persistent obstruction of pulmonary arteries by organized thrombi, leading to flow redistribution and secondary remodelling of the pulmonary microvascular bed. CTEPH has been reported with a cumulative incidence of between 0.1 and $9.1 \%$ in the first 2 years after a symptomatic PE event; the large margin of error is due to referral bias, the paucity of early symptoms, and the difficulty of differentiating acute PE from symptoms of pre-existing CTEPH $[439,440]$. A prospective, multicentre, observational screening survey for the detection of CTEPH included patients with acute PE from 11 centres in Switzerland, from March 2009 to November 2016. Screening for possible CTEPH was performed at 6,12 , and 24 months using a stepwise algorithm that included a phone-based dyspnoea survey, TTE, right heart catheterization, and radiological confirmation of CTEPH. Of 508 patients assessed for CTEPH screening over 2 years, CTEPH incidence following PE was 3.7 per 1000 patient-years, with a 2 year cumulative incidence of $0.79 \%$ [441]. In Germany, the incidence of CTEPH in 2016 was estimated at 5.7 per million adult population [442].

The hallmark of CTEPH is fibrotic transformation of a pulmonary arterial thrombus, causing fixed mechanical obstruction of pulmonary arteries and leading to overflow of the open pulmonary arterial bed. Together with collateral supply from systemic arteries downstream of pulmonary arterial occlusions, this contributes to microvascular remodelling causing a progressive increase in PVR [443]. Owing to this complex pathophysiology, there is no clear correlation between the degree of mechanical obstruction found at imaging and haemodynamics, which can deteriorate in the absence of recurrent PE [444]. 
TABLE 13 Risk factors and predisposing conditions for chronic thromboembolic pulmonary hypertension [447-449]

Findings related to the acute PE event (obtained at PE diagnosis)

Previous episodes of PE or DVT

Large pulmonary arterial thrombi on CTPA

Echocardiographic signs of PH/RV dysfunction ${ }^{\text {a }}$

CTPA findings suggestive of pre-existing chronic

thromboembolic disease ${ }^{\text {b }}$
Concomitant chronic diseases and conditions predisposing to CTEPH (documented at PE diagnosis or at 3-6 month follow-up)

Ventriculo-atrial shunts

Infected chronic i.v. lines or pacemakers

History of splenectomy

Thrombophilic disorders, particularly antiphospholipid antibody syndrome and high coagulation factor VIII levels

Non-O blood group

Hypothyroidism treated with thyroid hormones

History of cancer

Myeloproliferative disorders

Inflammatory bowel disease

Chronic osteomyelitis

CTEPH: chronic thromboembolic pulmonary hypertension; CTPA: computed tomographic pulmonary angiography; DVT: deep vein thrombosis; i.

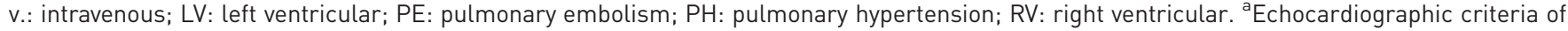
RV dysfunction are graphically presented in Figure 3, and their prognostic value summarized in supplementary table 3 . On CTPA (four-chamber views of the heart), RV dysfunction is defined as RV/LV diameter ratio $>1.0$. ${ }^{\text {b}}$ Direct and indirect vascular signs, as well as lung parenchymal findings, are summarized in supplementary table 2 .

Two historical trials assessed survival in patients with CTEPH before the availability of surgical treatment. In both studies, mean PAP $>30 \mathrm{mmHg}$ was related to poor survival, similar to that reported for idiopathic pulmonary arterial hypertension $[445,446]$.

The most frequently cited risk factors and predisposing conditions for CTEPH are shown in table 13. In an international registry, a history of acute PE was reported by $75 \%$ of patients [447]. Associated conditions and comorbidities included thrombophilic disorders, particularly antiphospholipid antibody syndrome and high coagulation factor VIII levels, cancer, a history of splenectomy, inflammatory bowel disease, ventriculo-atrial shunts, and infection of chronic i.v. lines and devices such as implantable pacemakers.

\subsubsection{Clinical presentation and diagnosis}

Diagnosing CTEPH is difficult. Algorithms for predicting [450] or ruling out CTEPH [451, 452] are limited by a lack of specificity. The clinical characteristics of patients enrolled in an international CTEPH registry have shown that the median age at diagnosis is 63 years and that both sexes are equally affected; paediatric cases are rare [447]. Clinical symptoms and signs are non-specific or absent in early CTEPH, with signs of right heart failure only becoming evident in advanced disease. Thus, early diagnosis remains a challenge in CTEPH, with a median time of 14 months between symptom onset and diagnosis in expert centres [453]. When present, the clinical symptoms of CTEPH may resemble those of acute PE or of pulmonary arterial hypertension; in the latter context, oedema and haemoptysis occur more often in CTEPH, while syncope is more common in pulmonary arterial hypertension [453].

The diagnosis of CTEPH is based on findings obtained after at least 3 months of effective anticoagulation, to distinguish this condition from acute PE. The diagnosis requires a mean PAP of $\geqslant 25 \mathrm{mmHg}$ along with a pulmonary arterial wedge pressure of $\leqslant 15 \mathrm{mmHg}$, documented at right heart catheterization in a patient with mismatched perfusion defects on V/Q lung scan. Specific diagnostic signs for CTEPH on multidetector CT angiography or conventional pulmonary cineangiography include ring-like stenoses, webs, slits, and chronic total occlusions [289].

Some patients may present with normal pulmonary haemodynamics at rest despite symptomatic disease. If other causes of exercise limitation are excluded, these patients are considered as having chronic thromboembolic disease (CTED). Identification of patients with chronic thromboembolism without $\mathrm{PH}$, who may have an indication for surgical or interventional treatment, requires particular expertise and should be done in CTEPH referral centres. Among 1019 patients who were submitted to pulmonary endarterectomy (PEA) in a UK referral centre, 42 patients did not have pulmonary hypertension at rest but showed functional improvement after the operation [454].

Planar V/Q lung scan is a suitable first-line imaging modality for CTEPH as it has $96-97 \%$ sensitivity and 90-95\% specificity for the diagnosis [455]. SPECT seems less sensitive than planar V/Q scanning if assessed at a level of individual segmental arteries, but it is unlikely to miss clinically relevant CTEPH in an individual patient. In contrast to CTEPH, abnormal mismatched perfusion defects sometimes found in 
pulmonary arterial hypertension and pulmonary veno-occlusive disease typically have a non-segmental pattern.

CTPA is gaining ground as a diagnostic modality in CTEPH [456], but it should not be used as a stand-alone test to exclude the disease [455]. Newer diagnostic tests include dual-energy CT, which allows the simultaneous assessment of patency of the pulmonary arteries and of lung perfusion, probably at a cost of some increase in radiation delivered to the patient. Magnetic resonance imaging of the pulmonary vasculature is still considered inferior to CT [457]. Cone-beam CT [458], angioscopy [459], intravascular ultrasound, and optical coherence tomography are more suitable for the characterization of lesions during interventional treatment than for diagnosis. High-resolution CT scan of the chest may assist in the differential diagnosis of CTEPH, showing emphysema, bronchial, or interstitial lung disease, as well as infarcts, and vascular and thoracic wall malformations. Perfusion inequalities manifesting as a mosaic parenchymal pattern are frequently found in CTEPH, but may also be observed in $\leqslant 12 \%$ of patients with other causes of PH. Differential diagnosis of CTEPH should also include pulmonary arteritis, pulmonary angiosarcoma, tumour embolism, parasites (hydatid cyst), foreign body embolism, and congenital or acquired pulmonary artery stenoses [289].

\subsubsection{Surgical treatment}

Surgical PEA is the treatment of choice for operable CTEPH. In contrast to surgical embolectomy for acute PE, treatment of CTEPH necessitates a true bilateral endarterectomy through the medial layer of the pulmonary arteries. It requires deep hypothermia and intermittent circulatory arrest, without a need for cerebral perfusion $[460,461]$. In-hospital mortality is currently as low as $4.7 \%$ [462] and is even lower in high-volume single centres [463]. The majority of patients experience substantial relief from symptoms and near-normalization of haemodynamics [461-464]. Owing to the complexity of both the surgical technique and peri-procedural management, PEA is performed in specialized centres. Eligibility for surgery requires a decision taken during a dedicated meeting of a multidisciplinary CTEPH team including experienced surgeons for PEA, interventional radiologists or cardiologists, radiologists experienced in pulmonary vascular imaging, and clinicians with expertise in PH. The CTEPH team should confirm the diagnosis, assess the surgical accessibility of chronic post-thrombotic obstructions ("surgical operability"), and consider the risks related to comorbidities ("medical operability"). The operability of patients with CTEPH is determined by multiple factors that cannot easily be standardized. These are related to the suitability of the patient, the expertise of the surgical team, and available resources. General criteria include pre-operative New York Heart Association (NYHA) functional class and the surgical accessibility of thrombi in the main, lobar, or segmental pulmonary arteries [462]. Advanced age per se is no contraindication for surgery. There is no haemodynamic threshold or measure of RV dysfunction that can be considered to preclude PEA.

Data from the international CTEPH registry, set up in 27 centres to evaluate the long-term outcome and outcome predictors in 679 operated and not-operated patients, showed estimated survival at 3 years of $89 \%$ in operated and 70\% in not-operated patients [465]. Mortality was associated with NYHA functional class, RA pressure, and a history of cancer [465]. In this prospective registry, the long-term prognosis of operated patients was better than the outcome of not-operated patients [465]. Additional correlates of mortality were bridging therapy with pulmonary vasodilators, post-operative $\mathrm{PH}$, surgical complications, and additional cardiac procedures in operated patients, and comorbidities such as coronary disease, left heart failure, and chronic obstructive pulmonary disease in not-operated patients [465]. A recent report identified mean PAP $\geqslant 38 \mathrm{mmHg}$ and PVR $\geqslant 425 \mathrm{dyn} \cdot \mathrm{s} \cdot \mathrm{cm}^{-5}$ as determinants of poor prognosis in survivors of surgical treatment for CTEPH [466].

Post-operative ECMO is recommended as the standard of care in PEA centres [461]. Early post-operative reperfusion oedema may require veno-arterial ECMO, and severe persistent $\mathrm{PH}$ may be bridged to emergency lung transplantation with ECMO. After PEA, patients should be followed in CTEPH centres to exclude persistent or recurrent $\mathrm{PH}$, with at least one haemodynamic assessment to be considered at 6-12 months after the intervention.

\subsubsection{Balloon pulmonary angioplasty}

Over the past decade, balloon pulmonary angioplasty (BPA) has emerged as an effective treatment for technically inoperable CTEPH. It allows dilatation of obstructions down to subsegmental vessels, which are inaccessible to surgery. BPA is a stepwise procedure requiring several (usually 4-10) separate sessions. This is necessary to engage all under-perfused lung segments, while limiting the contrast burden and radiation delivered per session. Navigation in distal pulmonary arteries requires particular expertise, as the complexity and individual variability of the pulmonary arterial tree greatly exceeds that of other vascular beds. Complications include wire- and balloon-induced injury, which may result in intrapulmonary 
bleeding, haemoptysis, and reperfusion lung injury. Usually, bleeding resolves spontaneously, but sometimes it has to be controlled by transient balloon inflation proximal to the site of perforation; in rare cases it requires embolization. Mild hypoxaemia is frequent and can be controlled by oxygen delivery. Mechanical ventilation or ECMO is rarely needed.

The largest published registry to date included 249 patients with a mean age of 61.5 years, who were treated with BPA between 2004 and 2013 in seven Japanese centres [467]. Mean PAP decreased from 43 to $24 \mathrm{mmHg}$ after terminating BPA sessions, and this result was maintained in 196 patients who underwent follow-up right heart catheterization. Complications occurred in $36 \%$ of the patients, including pulmonary injury (18\%), haemoptysis (14\%), and pulmonary artery perforation (2.9\%). After BPA, 30 day mortality was $2.6 \%$ and overall survival was $97 \%$ at 1 year [467].

While most of the BPA procedures are performed in technically inoperable patients, this method has also been used for sequential treatment for PH persisting after PEA. Few "rescue" BPA interventions performed in unstable patients remaining on ECMO after PEA were ineffective [468].

\subsubsection{Pharmacological treatment}

Optimal medical treatment for CTEPH consists of anticoagulants, as well as diuretics and oxygen in cases of heart failure or hypoxaemia. Lifelong oral anticoagulation with VKAs is recommended, and also after successful PEA or BPA. No data exist on the efficacy and safety of NOACs.

Pulmonary microvascular disease in CTEPH has provided the rationale for also testing drugs that have been approved for pulmonary arterial hypertension for this indication. Based on available data, medical treatment of CTEPH with targeted therapy is now justified for technically inoperable patients [469, 470], as well as for patients with PH persisting after PEA [469]. To date, the only drug approved for inoperable CTEPH or persistent/recurrent PH after PEA is riociguat, an oral stimulator of soluble guanylate cyclase [469]. In a prospective randomized trial of 261 patients with inoperable CTEPH or persistent/recurrent $\mathrm{PH}$ after PEA, treatment with riociguat significantly increased 6 min walking distance and reduced PVR [469]. In a similar population of 157 patients, the dual endothelin antagonist bosentan showed a positive effect on haemodynamics, but no improvement was observed in exercise capacity and the primary outcome was not met [471]. Another dual endothelin antagonist, macitentan, was found to significantly improve PVR and 6 min walking distance compared to placebo in a phase II trial focusing on inoperable patients with CTEPH [470]. Currently, riociguat is being tested in trials addressing its efficacy and safety: (i) as bridging therapy for patients scheduled to undergo PEA (NCT 03273257) and (ii) in comparison to BPA (NCT 02634203).

Overall, the effects on clinical worsening of drugs tested with RCTs in patients with CTEPH have not yet been clarified. Furthermore, no data exist on medical treatment in technically operable patients with prohibitive comorbidities or those refusing surgery. Off-label combination of drugs approved for pulmonary arterial hypertension has been proposed for CTEPH patients presenting with severe haemodynamic compromise, but only limited prospective data are available to date [470].

Medical therapy is not indicated in symptomatic survivors of acute PE with documented post-thrombotic obstructions but an absence of $\mathrm{PH}$ at right heart catheterization at rest (CTED).

\subsection{Strategies for patient follow-up after pulmonary embolism}

Figure 8 displays a proposed follow-up strategy for survivors of acute PE following discharge from hospital. Evaluation of the patients 3-6 months after the acute PE episode is recommended to assess the persistence (or new onset) and severity of dyspnoea or functional limitation, and to check for possible signs of VTE recurrence, cancer, or bleeding complications of anticoagulation. The severity of dyspnoea can be assessed using the Medical Research Council scale [160]; alternatively, the World Health Organization functional class can be determined (supplementary table 16) [289].

In patients complaining of persisting dyspnoea and poor physical performance, TTE should be considered as the next step to assess the probability of (chronic) PH and thus possible CTEPH. The criteria and levels of PH probability are defined by current ESC Guidelines [289], and are listed in supplementary tables 17 and 18. Patients with a high echocardiographic probability of $\mathrm{PH}$, or those with intermediate probability combined with elevated NT-proBNP levels or risk factors/predisposing conditions for CTEPH, such as those listed in table 13, should be considered for a V/Q scan.

If mismatched perfusion defects are found on the V/Q scan, referral to a PH or CTEPH expert centre for further diagnostic workup is indicated. If, on the other hand, the V/Q scan is normal and the patient's symptoms remain unexplained, CPET may be performed. By providing evidence of reduced maximal aerobic capacity, CPET supports the need for further follow-up visits and helps to identify candidates for 


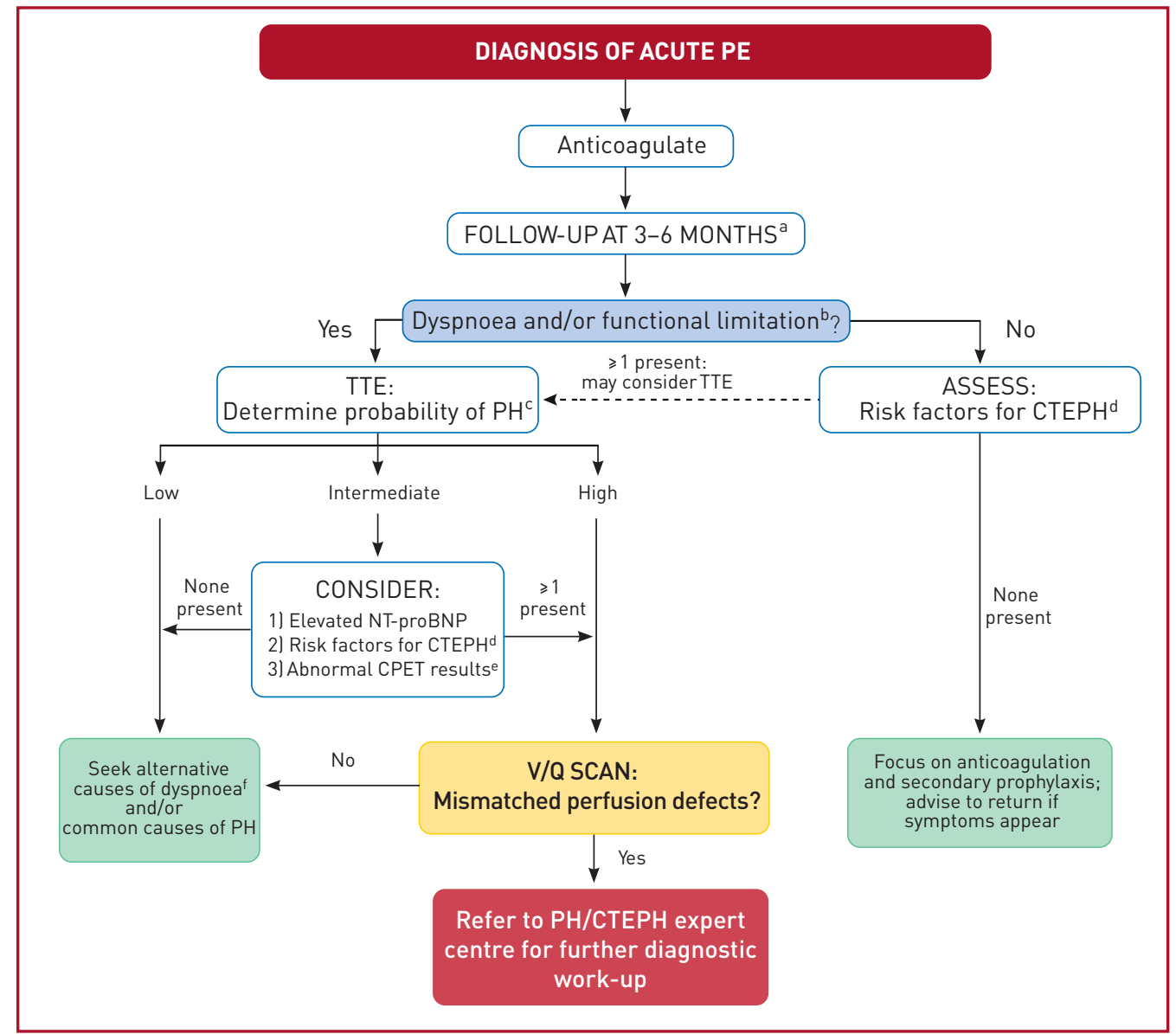

FIGURE 8 Follow-up strategy and diagnostic workup for long-term sequelae of pulmonary embolism. CPET: cardiopulmonary exercise testing; CTEPH: chronic thromboembolic pulmonary hypertension; NT-proBNP: $\mathrm{N}$-terminal pro B-type natriuretic peptide; PE: pulmonary embolism; PH: pulmonary hypertension; TTE: transthoracic echocardiography/echocardiogram; V/Q: ventilation/perfusion (lung scintigraphy). ${ }^{a}$ Assess the persistence (or new onset) and severity of dyspnoea or functional limitation, and also check for possible signs

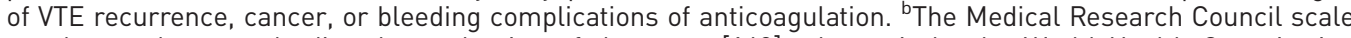
can be used to standardize the evaluation of dyspnoea [160]; alternatively, the World Health Organization functional class can be determined (supplementary table 16) [289]. 'As defined by the ESC/ERS guidelines on the diagnosis and treatment of Pulmonary Hypertension (supplementary tables 17 and 18) [289]. ${ }^{\text {d Risk factors }}$ and predisposing conditions for CTEPH are listed in table 13. ${ }^{e}$ Cardiopulmonary exercise testing, if appropriate expertise and resources are available on site; abnormal results include, among others, reduced maximal aerobic capacity (peak oxygen consumption), reduced ventilatory equivalent for carbon dioxide, and reduced end-tidal carbon dioxide pressure. ${ }^{\mathrm{f}}$ Consider CPET in the diagnostic work-up.

pulmonary rehabilitation, exercise, or weight-reduction programmes $[435,436]$. CPET may also be helpful in patients with suspected CTEPH and coexisting left heart and/or respiratory disease; in such cases, it can help to establish the main limiting factor and thus set priorities for the treatment strategy [472].

For patients who report as free of dyspnoea or functional limitation at 3-6-month follow-up after acute PE but have risk factors/predisposing conditions for CTEPH (table 13), further follow-up visits may be scheduled and the patient must be advised to return if symptoms appear. Alternatively, TTE may be considered to assess the probability of $\mathrm{PH}$ (figure 8).

Apart from the recommended screening and diagnostic measures, an integrated model of patient care after PE should be provided, taking into consideration the infrastructure and possibilities offered by each country's health system. The model should include appropriately qualified nurses, interdisciplinary working with physicians in the care of both in-hospital and ambulatory PE patients, standardized treatment protocols adapted to the capacities of each hospital, and bidirectional referral pathways between general practice and the hospital. Such models ensure smooth transitions between hospital specialists and practitioners; provide continuity, and easy access to care along with information and education; and respect the patients' preferences, and those of their families and social environment. In this context, 
nurse-led care models to deliver follow-up have been shown to be effective after acute coronary syndrome [473], in primary care-based management of chronic diseases [474], and in community based self-management initiatives [475]. A recently published study investigated the care of 42 patients followed at a pulmonary arterial hypertension $(\mathrm{PAH}) / \mathrm{CTEPH}$ nurse-led outpatient clinic and showed positive results [476]. During patient follow-up visits, appropriately qualified nurses screen for signs and symptoms indicating VTE recurrence or complications of treatment, and assess adherence to medication. Nurses work collaboratively with patients using behavioural frameworks and motivational interviewing, to identify and modify associated risk factors (smoking cessation, diet, physical activity, and exercise). In addition, they promote self-management skills such as the use of compression stockings, safe increase in mobility, increased awareness of signs of recurrence, or complications.

\subsection{Recommendations for follow-up after acute pulmonary embolism}

\section{Recommendations}

Routine clinical evaluationc of patients 3-6 months after the acute PE episode is recommended [288, 352, 353, 437].

An integrated model of patient care after PE linvolving hospital specialists, appropriately qualified nurses, and primary care physicians) is recommended to ensure optimal transition from hospital to community care.

In symptomatic patients with mismatched perfusion defects persisting on $\mathrm{V} / \mathrm{Q}$ scand beyond 3 months after acute PE, referral to a PH/CTEPH expert centre is recommended, after taking into account the results of echocardiography, natriuretic peptide levels, and/or CPET [477].

Further diagnostic evaluatione should be considered in patients with persistent or new-onset dyspnoea/exercise limitation after PE.

Further diagnostic evaluatione may be considered in asymptomatic patients with risk factors for CTEPHf $[447-449,478]$.
Class $^{\mathrm{a}}$ Level $^{\mathrm{b}}$

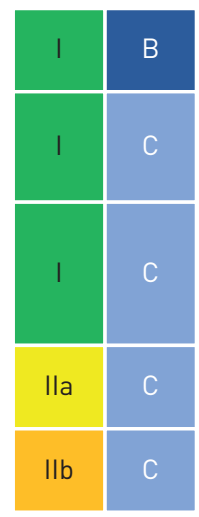

CPET: cardiopulmonary exercise testing; CT: computed tomography; CTEPH: chronic thromboembolic pulmonary hypertension; PE: pulmonary embolism; PH: pulmonary hypertension; V/Q: ventilation/perfusion (lung scintigraphy). ${ }^{a}$ Class of recommendation. ${ }^{b}$ Level of evidence. ${ }^{C}$ For symptoms suggesting recurrence, bleeding, malignancy, or persistent or new-onset exercise limitation, and to decide on extension of anticoagulant treatment. 'Alternatively, dual-energy CT may be used, if appropriate expertise and resources are available on-site. ${ }^{\mathrm{e}} \mathrm{As}$ proposed in the algorithm shown in figure $8 .{ }^{\mathrm{f}}$ Risk factors and predisposing conditions for CTEPH are listed in table 13

\section{Non-thrombotic pulmonary embolism}

This section is included in the supplementary data.

\section{Key messages}

The ESC Task Force has selected 10 simple key messages and rules to guide physicians in the diagnosis and management of PE:

1) In patients presenting with haemodynamic instability, perform bedside TTE as a fast, immediate step to differentiate suspected high-risk PE from other acute life-threatening situations.

2) If you suspect acute PE, institute anticoagulation therapy as soon as possible, while the diagnostic workup is ongoing, unless the patient is bleeding or has absolute contraindications to this therapy.

3) Use recommended, validated diagnostic algorithms for PE, including standardized assessment of (pre-test) clinical probability and D-dimer testing. They help to avoid unnecessary, expensive, and potentially harmful imaging tests and exposure to ionizing radiation.

4) If the CTPA report suggests single subsegmental PE, consider the possibility of a false-positive finding. Discuss the findings again with the radiologist and/or seek a second opinion to avoid misdiagnosis, and unnecessary, potentially harmful anticoagulation treatment.

5) Confirmation of PE in a patient, without haemodynamic instability, must be followed by further risk assessment involving clinical findings, evaluation of the size and/or function of the RV, and laboratory biomarkers as appropriate. This information will help you to decide on the need for reperfusion treatment or monitoring for patients at elevated risk, or consider the option of early discharge and continuation of anticoagulation on an ambulatory basis for patients at low risk.

6) As soon as you diagnose (or strongly suspect) high-risk PE, select the best reperfusion option (systemic thrombolysis, surgical embolectomy, or catheter-directed treatment) considering the 
patient's risk profile, and the resources and expertise available at your hospital. For patients with intermediate-high-risk PE, reperfusion is not first-line treatment, but you should prospectively plan the management strategy with your team to have a contingency plan ready if the situation deteriorates.

7) Prefer anticoagulation with a NOAC over the "traditional" LMWH-VKA regimen unless the patient has contraindication(s) to this type of drug.

8) Always remember that, with the exception of acute PE provoked by a strong transient/reversible risk factor, there is a lifelong risk of VTE recurrence after a first episode of PE. Consequently, re-examine the patient after the first 3-6 months of anticoagulation, weigh the benefits versus risks of continuing treatment, and decide on the extension and dose of anticoagulant therapy, also considering the patient's preference. Remember to recommend regular follow-up examinations, e.g. at yearly intervals.

9) If you suspect PE in a pregnant patient, consider diagnostic pathways and algorithms including CTPA or V/Q lung scan, which can be used safely during pregnancy.

10) After acute PE, patients should not be lost to follow-up. Apart from checking for possible signs of VTE recurrence, cancer, or bleeding complications of anticoagulation, ask the patient if there is persisting or new-onset dyspnoea or functional limitation. If yes, implement a staged diagnostic workup to exclude CTEPH or chronic thromboembolic disease, and to detect/treat comorbidity or "simple" deconditioning. Follow-up imaging is not routinely recommended in an asymptomatic patient, but it may be considered in patients with risk factors for development of CTEPH.

\section{Gaps in the evidence}

\subsection{Diagnosis}

- The optimal method to adjust (based on the patient's age or in combination with clinical probability) the $\mathrm{D}$-dimer threshold, permitting the exclusion of PE while reducing the number of unnecessary imaging tests to a minimum, remains to be determined.

- The diagnostic value and clinical significance of isolated subsegmental contrast-filling defects in the modern CTPA era remain controversial.

- No robust data exist to guide the decision on whether to treat incidental PE with anticoagulants compared with a strategy of watchful waiting.

- For patients presenting with non-traumatic chest pain, the benefits versus risks of "triple rule-out" (for coronary artery disease, PE, and aortic dissection) CT angiography need further evaluation before such an approach can be routinely recommended.

\subsection{Assessment of pulmonary embolism severity and the risk of early death}

- The optimal, clinically most relevant combination (and cut-off levels) of clinical and biochemical predictors of early PE-related death remain to be determined, particularly with regard to identifying possible candidates for reperfusion treatment among patients with intermediate-risk PE.

- The need for assessment of the RV status in addition to clinical parameters, to classify a patient with acute symptomatic PE as being at low versus intermediate risk, needs to be confirmed by further prospective management (cohort) studies.

\subsection{Treatment in the acute phase}

- The clinical benefits versus risks of reduced-dose thrombolysis and catheter-based reperfusion modalities in patients with intermediate-high-risk PE should be evaluated in prospective randomized trials.

- The place of ECMO in the management of acute high-risk PE awaits support by additional evidence from prospective management (cohort) studies.

- The optimal anticoagulant $\operatorname{drug}(\mathrm{s})$ and regimen in patients with renal insufficiency and $\mathrm{CrCl}$ $<30 \mathrm{~mL} / \mathrm{min}$ remain unclear.

- The criteria for selecting patients for early discharge and outpatient treatment of PE, and particularly the need for assessment of the RV status with imaging methods and/or laboratory markers in addition to calculating a clinical score, need to be further validated in prospective cohort studies.

\subsection{Chronic treatment and prevention of recurrence}

- The clinical value and the possible therapeutic implications of models or scores assessing the risk of VTE recurrence, and the risk of bleeding under anticoagulation, need to be revisited in the NOAC era.

- The effectiveness of extended treatment with a reduced dose, or apixaban or rivaroxaban, should be confirmed in patients with a high risk of recurrent PE. 
- The evidence supporting the efficacy and safety of NOACs for the treatment of PE in patients with cancer needs to be extended by further studies.

- In patients with cancer, the anticoagulant regimen and dose after the first 6 months should be clarified and prospectively tested.

- The optimal time for discontinuing anticoagulant treatment after an episode of acute PE in patients with cancer is yet to be determined.

\subsection{Pulmonary embolism and pregnancy}

- Diagnostic algorithms for PE in pregnancy, using modern radiological imaging techniques and low radiation doses, need to be prospectively tested in adequately powered cohort studies.

- Controversy persists on the optimal LMWH dose and regimen for the treatment of PE during pregnancy.

- NOACs are not allowed in pregnancy. However, if exposure to these drugs occurs during pregnancy despite this warning, any possible effects on the fetus should be recorded to provide more precise information on the risks and complications of these drugs, and adapt the instructions to physicians in the future.

\subsection{Long-term sequelae of pulmonary embolism}

- The optimal follow-up strategy, including the spectrum of diagnostic tests that may be necessary, in patients with persisting symptoms and functional limitation after acute PE needs to be defined and prospectively validated.

- In the absence of persisting symptoms or functional limitation after acute PE, the criteria for identifying patients whose risk of developing CTEPH may be sufficiently high to justify further diagnostic workup require further elaboration and validation in prospective cohort studies.

\section{4. "What to do" and "what not to do" messages from the Guidelines}

\section{Diagnosis}

In suspected high-risk PE, perform bedside echocardiography or emergency CTPA (depending on availability and clinical circumstances) for diagnosis.

In suspected high-risk PE, initiate intravenous anticoagulation with UFH without delay, including a weight-adjusted bolus injection.

In suspected PE without haemodynamic instability, use validated diagnostic criteria.

In suspected PE without haemodynamic instability, initiate anticoagulation in case of high or intermediate clinical probability, while diagnostic workup is in progress.

Base the diagnostic strategy on clinical probability, using either clinical judgement or a validated prediction rule.

Measure D-dimers in plasma, preferably with a highly sensitive assay, in outpatients/emergency department patients with low or intermediate clinical probability, or who are PE-unlikely.

Reject the diagnosis of PE (without further testing) if CTPA is normal in a patient with low or intermediate clinical probability, or if the patient is PE-unlikely.

Reject the diagnosis of PE (without further testing) if the perfusion lung scan is normal.

Accept the diagnosis of PE if CTPA shows a segmental or more proximal filling defect in a patient with intermediate or high clinical probability.

Accept the diagnosis of VTE if CUS shows a proximal DVT in a patient with clinical suspicion of PE.

Do not measure D-dimers in patients with high clinical probability, as a normal result does not safely exclude PE.

Do not perform CT venography as an adjunct to CTPA.

Do not perform MRA to rule out PE.

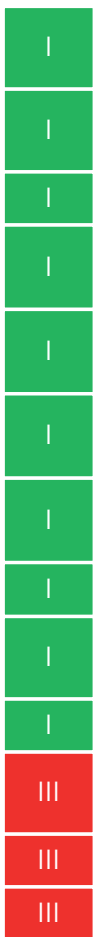

\section{Risk assessment}

Stratify patients with suspected or confirmed PE, based on the presence of haemodynamic instability, to identify those at high risk of early mortality.

In patients without haemodynamic instability, further stratify PE into intermediate- and low-risk categories. 


\section{Treatment in the acute phase}

Administer systemic thrombolytic therapy to patients with high-risk PE.

Surgical pulmonary embolectomy for patients with high-risk PE, in whom recommended thrombolysis is contraindicated or has failed.

If anticoagulation is initiated parenterally in a patient without haemodynamic instability, prefer LMWH or fondaparinux over UFH.

When oral anticoagulation is initiated in a patient with PE who is eligible for a NOAC lapixaban, dabigatran, edoxaban, or rivaroxaban), prefer a NOAC.

As an alternative to a NOAC, administer a VKA, overlapping with parenteral anticoagulation until an INR of 2.5 (range 2.0-3.0) has been reached.

Administer rescue thrombolytic therapy to a patient with haemodynamic deterioration on anticoagulation treatment.

Do not use NOACs in patients with severe renal impairment or in those with antiphospholipid antibody syndrome.

Do not routinely administer systemic thrombolysis as primary treatment in patients with

intermediate- or low-risk PE.

Do not routinely use inferior vena cava filters.

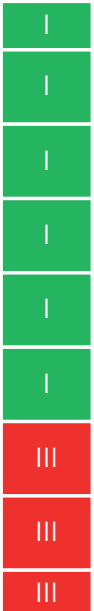

\section{Chronic treatment and prevention of recurrence}

Administer therapeutic anticoagulation for $\geqslant 3$ months to all patients with $\mathrm{PE}$.

Discontinue therapeutic oral anticoagulation after 3 months in patients with first PE secondary to a major transient/reversible risk factor.

Continue oral anticoagulant treatment indefinitely in patients presenting with recurrent VTE lat least one previous episode of PE or DVT) that is not related to a major transient or reversible risk factor.

Continue oral anticoagulant treatment with a VKA indefinitely in patients with antiphospholipid antibody syndrome.

In patients who receive extended anticoagulation, reassess drug tolerance and adherence, hepatic and renal function, and the bleeding risk at regular intervals.

\section{$\mathrm{PE}$ in pregnancy}

Perform formal diagnostic assessment with validated methods if PE is suspected during pregnancy or in the post-partum period.

Administer therapeutic, fixed doses of LMWH, based on early pregnancy weight, in the majority of pregnant women without haemodynamic instability.

Do not insert a spinal or epidural needle within $24 \mathrm{~h}$ since the last LMWH dose.

Do not administer LMWH within $4 \mathrm{~h}$ of removal of an epidural catheter.

Do not use NOACs during pregnancy or lactation.

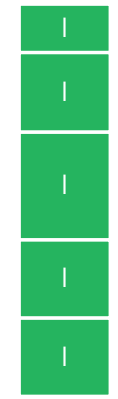

\section{Post-PE care and long-term sequelae}

Routinely re-evaluate patients 3-6 months after acute PE.

Implement an integrated model of care after acute $\mathrm{PE}$, in order to ensure optimal transition from hospital to ambulatory care.

Refer symptomatic patients with mismatched perfusion defects on V/Q lung scan beyond 3 months after acute PE to a pulmonary hypertension/CTEPH expert centre, taking into account the results of echocardiography, natriuretic peptide, and/or cardiopulmonary exercise testing.

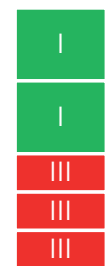

CT: computed tomography; CTPA: computed tomographic pulmonary angiography/angiogram; CTEPH: chronic thromboembolic pulmonary hypertension; CUS: compression ultrasonography; DVT: deep vein thrombosis; INR: international normalized ratio; LMWH: low-molecular weight heparin; MRA: magnetic resonance angiography; NOAC(s): non-vitamin $\mathrm{K}$ antagonist oral anticoagulant(s); PE: pulmonary embolism; UFH: unfractionated heparin; VKA: vitamin $\mathrm{K}$ antagonist; V/Q: ventilation/perfusion llung

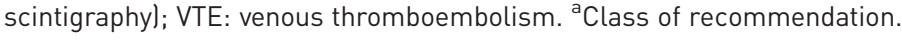

\section{Appendix}

Author/Task Force Member Affiliations: Cecilia Becattini, Internal and Cardiovascular Medicine, University of Perugia, Perugia, Italy; Héctor Bueno, Centro Nacional de Investigaciones Cardiovasculares, Madrid, Spain; and Cardiology,

Hospital Universitario 12 de Octubre \& i+12 Research Institute, Madrid, Spain; CIBERCV, Madrid, Spain; Geert-Jan Geersing, Julius Center for Health Sciences and Primary Care, University Medical Center Utrecht, Utrecht University, Utrecht, Netherlands; Veli-Pekka Harjola, Emergency Medicine, Department of Emergency Medicine and Services, Helsinki University, Helsinki University Hospital, Helsinki, Finland; Menno V. Huisman, Thrombosis and Hemostasis, Leiden University Medical Center, Leiden, Netherlands; Marc Humbert, Service de Pneumologie, Hôpital Bicêtre, 
Assistance Publique-Hôpitaux de Paris, Univ. Paris-Sud, Université Paris-Saclay, Le Kremlin-Bicêtre, France; Catriona Sian Jennings, National Heart and Lung Institute (NHLI), Imperial College London, London, United Kingdom; David Jiménez, Respiratory Department, Ramón y Cajal Hospital and Alcala University, IRYCIS, Madrid, Spain; Nils Kucher, Angiology, University Hospital, Zurich, Switzerland; Irene Marthe Lang, Cardiology, Medical University of Vienna, Vienna, Austria; Mareike Lankeit, Department of Internal Medicine and Cardiology, Campus Virchow Klinikum, Charité-University Medicine Berlin, Berlin, Germany; and Center for Thrombosis and Hemostasis, University Medical Center Mainz, Mainz, Germany; Clinic of Cardiology and Pneumology, University Medical Center Göttingen, Göttingen, Germany; Roberto Lorusso, Cardio-Thoracic Surgery Department, Heart and Vascular Centre, Maastricht University Medical Centre (MUMC), Cardiovascular Research Institute Maastricht (CARIM), Maastricht, Netherlands: Lucia Mazzolai, Department of Angiology, CHUV, Lausanne, Switzerland; Nicolas Meneveau, Department of Cardiology, University Hospital Jean Minjoz and EA3920, University of Franche-Comté, Besançon, France; Fionnuala Ní Áinle, Haematology, Rotunda and Mater University Hospitals, Dublin, University College Dublin, Dublin, Ireland; Paolo Prandoni, Arianna Foundation on Anticoagulation, Bologna, Italy; Piotr Pruszczyk, Department of Internal Medicine and Cardiology, Medical University of Warsaw, Warsaw, Poland; Marc Righini, Division of Angiology and Hemostasis, Geneva University Hospitals and Faculty of Medicine, Geneva, Switzerland; Adam Torbicki, Department of Pulmonary Circulation, Thromboembolic Diseases and Cardiology, Centre of Postgraduate Medical Education, Warsaw, ECZ-Otwock, Poland; Eric Van Belle, Cardiology, Institut Coeur Poumon CHU de Lille and INSERM U1011 Lille, Lille, France; José Luis Zamorano, Cardiology, Hospital Ramón y Cajal, Madrid, Spain.

ESC Committee for Practice Guidelines (CPG): Stephan Windecker (Chairperson) (Switzerland), Victor Aboyans (France), Colin Baigent (United Kingdom), Jean-Philippe Collet (France), Veronica Dean (France), Victoria Delgado (Netherlands), Donna Fitzsimons (United Kingdom), Chris P. Gale (United Kingdom), Diederick E. Grobbee (Netherlands), Sigrun Halvorsen (Norway), Gerhard Hindricks (Germany), Bernard Iung (France), Peter Jüni (Canada), Hugo A. Katus (Germany), Ulf Landmesser (Germany), Christophe Leclercq (France), Maddalena Lettino (Italy), Basil S. Lewis (Israel), Bela Merkely (Hungary), Christian Mueller (Switzerland), Steffen E. Petersen (United Kingdom), Anna Sonia Petronio (Italy), Dimitrios J. Richter (Greece), Marco Roffi (Switzerland), Evgeny Shlyakhto (Russian Federation), Iain A. Simpson (United Kingdom), Miguel Sousa-Uva (Portugal), Rhian M. Touyz (United Kingdom).

ESC National Cardiac Societies actively involved in the review process of the 2019 ESC Guidelines on the diagnosis and management of acute pulmonary embolism: Algeria: Algerian Society of Cardiology, Naima Hammoudi; Armenia: Armenian Cardiologists Association, Hamlet Hayrapetyan; Austria: Austrian Society of Cardiology, Julia Mascherbauer; Azerbaijan: Azerbaijan Society of Cardiology, Firdovsi Ibrahimov; Belarus: Belorussian Scientific Society of Cardiologists, Oleg Polonetsky; Belgium: Belgian Society of Cardiology, Patrizio Lancellotti; Bulgaria: Bulgarian Society of Cardiology, Mariya Tokmakova; Croatia: Croatian Cardiac Society, Bosko Skoric; Cyprus: Cyprus Society of Cardiology, Ioannis Michaloliakos; Czech Republic: Czech Society of Cardiology, Martin Hutyra; Denmark: Danish Society of Cardiology, Søren Mellemkjaer; Egypt: Egyptian Society of Cardiology, Mansour Mostafa; Estonia: Estonian Society of Cardiology, Julia Reinmets; Finland: Finnish Cardiac Society, Pertti Jääskeläinen; France: French Society of Cardiology, Denis Angoulvant; Germany: German Cardiac Society, Johann Bauersachs; Greece: Hellenic Society of Cardiology, George Giannakoulas; Hungary: Hungarian Society of Cardiology, Endre Zima; Italy: Italian Federation of Cardiology, Carmine Dario Vizza; Kazakhstan: Association of Cardiologists of Kazakhstan, Akhmetzhan Sugraliyev; Kosovo (Republic of): Kosovo Society of Cardiology, Ibadete Bytyçi; Latvia: Latvian Society of Cardiology, Aija Maca; Lithuania: Lithuanian Society of Cardiology, Egle Ereminiene; Luxembourg: Luxembourg Society of Cardiology, Steve Huijnen; Malta: Maltese Cardiac Society, Robert Xuereb; Moldova (Republic of): Moldavian Society of Cardiology, Nadejda Diaconu; Montenegro: Montenegro Society of Cardiology, Nebojsa Bulatovic; Morocco: Moroccan Society of Cardiology, Ilyasse Asfalou; North Macedonia: North Macedonian Society of Cardiology, Marijan Bosevski; Norway: Norwegian Society of Cardiology, Sigrun Halvorsen; Poland: Polish Cardiac Society, Bożena Sobkowicz; Portugal: Portuguese Society of Cardiology, Daniel Ferreira; Romania: Romanian Society of Cardiology, Antoniu Octavian Petris; Russian Federation: Russian Society of Cardiology, Olga Moiseeva; San Marino: San Marino Society of Cardiology, Marco Zavatta; Serbia: Cardiology Society of Serbia, Slobodan Obradovic; Slovakia: Slovak Society of Cardiology, Iveta Šimkova; Slovenia: Slovenian Society of Cardiology, Peter Radsel; Spain: Spanish Society of Cardiology, Borja Ibanez; Sweden: Swedish Society of Cardiology, Gerhard Wikström; Switzerland: Swiss Society of Cardiology, Drahomir Aujesky; Turkey: Turkish Society of Cardiology, Cihangir Kaymaz; Ukraine: Ukrainian Association of Cardiology, Alexander Parkhomenko; United Kingdom of Great Britain and Northern Ireland: British Cardiovascular Society, Joanna Pepke-Zaba.

\section{References}

$1 \quad$ Mazzolai L, Aboyans V, Ageno W, et al. Diagnosis and management of acute deep vein thrombosis: a joint consensus document from the European Society of Cardiology working groups of aorta and peripheral vascular diseases and pulmonary circulation and right ventricular function. Eur Heart $J$ 2018; 39: 4208-4218.

2 Raskob GE, Angchaisuksiri P, Blanco AN, et al. Thrombosis: a major contributor to global disease burden. Arterioscler Thromb Vasc Biol 2014; 34: 2363-2371.

3 Wendelboe AM, Raskob GE. Global burden of thrombosis: epidemiologic aspects. Circ Res 2016; 118: $1340-1347$.

4 Keller K, Hobohm L, Ebner M, et al. Trends in thrombolytic treatment and outcomes of acute pulmonary embolism in Germany. Eur Heart J 2019; in press [https://doi.org/10.1093/eurheartj/ehz236].

5 de Miguel-Diez J, Jimenez-Garcia R, Jimenez D, et al. Trends in hospital admissions for pulmonary embolism in Spain from 2002 to 2011. Eur Respir J 2014; 44: 942-950.

6 Dentali F, Ageno W, Pomero F, et al. Time trends and case fatality rate of in-hospital treated pulmonary embolism during 11 years of observation in Northwestern Italy. Thromb Haemost 2016; 115: 399-405.

7 Lehnert $\mathrm{P}$, Lange $\mathrm{T}$, Moller $\mathrm{CH}$, et al. Acute pulmonary embolism in a national Danish cohort: increasing incidence and decreasing mortality. Thromb Haemost 2018; 118: 539-546.

8 Barco S, Woersching AL, Spyropoulos AC, et al. European Union-28: an annualised cost-of-illness model for venous thromboembolism. Thromb Haemost 2016; 115: 800-808.

9 Cohen AT, Agnelli G, Anderson FA, et al. Venous thromboembolism (VTE) in Europe. The number of VTE events and associated morbidity and mortality. Thromb Haemost 2007; 98: 756-764. 
Jimenez D, de Miguel-Diez J, Guijarro R, et al. Trends in the management and outcomes of acute pulmonary embolism: analysis from the RIETE registry. J Am Coll Cardiol 2016; 67: 162-170.

Agarwal S, Clark D III, Sud K, et al. Gender disparities in outcomes and resource utilization for acute pulmonary embolism hospitalizations in the United States. Am J Cardiol 2015; 116: 1270-1276.

Roy PM, Meyer G, Vielle B, et al. Appropriateness of diagnostic management and outcomes of suspected pulmonary embolism. Ann Intern Med 2006; 144: 157-164.

Jimenez D, Bikdeli B, Barrios D, et al. Management appropriateness and outcomes of patients with acute pulmonary embolism. Eur Respir J 2018; 51: 1800445.

Wiener RS, Schwartz LM, Woloshin S. Time trends in pulmonary embolism in the United States: evidence of overdiagnosis. Arch Intern Med 2011; 171: 831-837.

Shiraev TP, Omari A, Rushworth RL. Trends in pulmonary embolism morbidity and mortality in Australia. Thromb Res 2013; 132: 19-25.

Tsai J, Grosse SD, Grant AM, et al. Trends in in-hospital deaths among hospitalizations with pulmonary embolism. Arch Intern Med 2012; 172: 960-961.

Yang Y, Liang L, Zhai Z, et al. Pulmonary embolism incidence and fatality trends in chinese hospitals from 1997 to 2008: a multicenter registration study. PLoS One 2011; 6: e26861. Cardiol 2016; 67: 976-990.

TI, Brandao LR, Kahr WH, et al. Clinical features and outcome of pulmonary embolism in children. $\mathrm{Br}$ Haematol 2008; 142: 808-818.

Andrew M, David M, Adams M, et al. Venous thromboembolic complications (VTE) in children: first analyses of the Canadian Registry of VTE. Blood 1994; 83: 1251-1257.

Stein PD, Kayali F, Olson RE. Incidence of venous thromboembolism in infants and children: data from the National Hospital Discharge Survey. J Pediatr 2004; 145: 563-565.

van Ommen $\mathrm{CH}$, Heijboer $\mathrm{H}$, Buller HR, et al. Venous thromboembolism in childhood: a prospective two-year registry in The Netherlands. J Pediatr 2001; 139: 676-681.

Rogers MA, Levine DA, Blumberg N, et al. Triggers of hospitalization for venous thromboembolism. Circulation 2012; 125: 2092-2099.

Anderson FA Jr, Spencer FA. Risk factors for venous thromboembolism. Circulation 2003; 107: I9-I16.

$\mathrm{Ku} \mathrm{GH}$, White RH, Chew HK, et al. Venous thromboembolism in patients with acute leukemia: incidence, risk factors, and effect on survival. Blood 2009; 113: 3911-3917.

Chew HK, Wun T, Harvey D, et al. Incidence of venous thromboembolism and its effect on survival among patients with common cancers. Arch Intern Med 2006; 166: 458-464.

Timp JF, Braekkan SK, Versteeg HH, et al. Epidemiology of cancer-associated venous thrombosis. Blood 2013; 122: $1712-1723$.

Blom JW, Doggen CJ, Osanto S, et al. Malignancies, prothrombotic mutations, and the risk of venous thrombosis. JAMA 2005; 293: 715-722.

Gussoni G, Frasson S, La Regina M, et al. Three-month mortality rate and clinical predictors in patients with venous thromboembolism and cancer. Findings from the RIETE registry. Thromb Res 2013; 131: 24-30.

Blanco-Molina A, Rota L, Di Micco P, et al. Venous thromboembolism during pregnancy, postpartum or during contraceptive use. Thromb Haemost 2010; 103: 306-311.

Blanco-Molina A, Trujillo-Santos J, Tirado R, et al. Venous thromboembolism in women using hormona contraceptives. Findings from the RIETE Registry. Thromb Haemost 2009; 101: 478-482.

van Hylckama Vlieg A, Middeldorp S. Hormone therapies and venous thromboembolism: where are we now? J Thromb Haemost 2011; 9: 257-266.

Lidegaard $\varnothing$, Nielsen LH, Skovlund CW, et al. Risk of venous thromboembolism from use of oral contraceptives containing different progestogens and oestrogen doses: Danish cohort study, 2001-9. BMJ 2011; 343: d6423.

de Bastos M, Stegeman BH, Rosendaal FR, et al. Combined oral contraceptives: venous thrombosis. Cochrane Database Syst Rev 2014; 3: CD010813.

van Vlijmen EF, Wiewel-Verschueren S, Monster TB, et al. Combined oral contraceptives, thrombophilia and the risk of venous thromboembolism: a systematic review and meta-analysis. J Thromb Haemost 2016; 14: 1393-1403.

Tricotel A, Collin C, Zureik M. Impact of the sharp changes in the use of contraception in 2013 on the risk of pulmonary embolism in France. J Thromb Haemost 2015; 13: 1576-1580.

Kemmeren JM, Algra A, Grobbee DE. Third generation oral contraceptives and risk of venous thrombosis: meta-analysis. BMJ 2001; 323: 131-134.

van Hylckama Vlieg A, Helmerhorst FM, Rosendaal FR. The risk of deep venous thrombosis associated with injectable depot-medroxyprogesterone acetate contraceptives or a levonorgestrel intrauterine device. Arterioscler Thromb Vasc Biol 2010; 30: 2297-2300.

Sweetland S, Beral V, Balkwill A, et al. Venous thromboembolism risk in relation to use of different types of postmenopausal hormone therapy in a large prospective study. J Thromb Haemost 2012; 10: 2277-2286.

Clayton TC, Gaskin M, Meade TW. Recent respiratory infection and risk of venous thromboembolism: case-control study through a general practice database. Int J Epidemiol 2011; 40: 819-827.

Smeeth L, Cook C, Thomas S, et al. Risk of deep vein thrombosis and pulmonary embolism after acute infection in a community setting. Lancet 2006; 367: 1075-1079. chemotherapy-associated thrombosis. Blood 2008; 111: 4902-4907.

Dijk FN, Curtin J, Lord D, et al. Pulmonary embolism in children. Paediatr Respir Rev 2012; 13: 112-122.

Piazza G, Goldhaber SZ. Venous thromboembolism and atherothrombosis: an integrated approach. Circulation 2010; 121: 2146-2150.

Steffen LM, Cushman M, Peacock JM, et al. Metabolic syndrome and risk of venous thromboembolism longitudinal investigation of thromboembolism etiology. J Thromb Haemost 2009; 7: 746-751.

Severinsen MT, Kristensen SR, Johnsen SP, et al. Anthropometry, body fat, and venous thromboembolism: a Danish follow-up study. Circulation 2009; 120: 1850-1857. 
Ageno W, Becattini C, Brighton $\mathrm{T}$, et al. Cardiovascular risk factors and venous thromboembolism: a meta-analysis. Circulation 2008; 117: 93-102.

48 Piazza G, Goldhaber SZ, Lessard DM, et al. Venous thromboembolism in patients with symptomatic atherosclerosis. Thromb Haemost 2011; 106: 1095-1102.

49 Montecucco F, Mach F. Should we focus on "venous vulnerability" instead of "plaque vulnerability" in symptomatic atherosclerotic patients? Thromb Haemost 2011; 106: 995-996.

50 Gresele P, Momi S, Migliacci R. Endothelium, venous thromboembolism and ischaemic cardiovascular events. Thromb Haemost 2010; 103: 56-61.

51 Fox EA, Kahn SR. The relationship between inflammation and venous thrombosis - A systematic review of clinical studies. Thromb Haemost 2005; 94: 362-365.

52 Wattanakit K, Lutsey PL, Bell EJ, et al. Association between cardiovascular disease risk factors and occurrence of venous thromboembolism. A time-dependent analysis. Thromb Haemost 2012; 108: 508-515.

53 Enga KF, Braekkan SK, Hansen-Krone IJ, et al. Cigarette smoking and the risk of venous thromboembolism: the Tromsø Study. J Thromb Haemost 2012; 10: 2068-2074.

54 Sorensen HT, Horvath-Puho E, Lash TL, et al. Heart disease may be a risk factor for pulmonary embolism without peripheral deep venous thrombosis. Circulation 2011; 124: 1435-1441.

55 Prandoni P, Pesavento R, Sorensen HT, et al. Prevalence of heart diseases in patients with pulmonary embolism with and without peripheral venous thrombosis: findings from a cross-sectional survey. Eur J Intern Med 2009; 20: 470-473.

56 Sorensen HT, Horvath-Puho E, Pedersen L, et al. Venous thromboembolism and subsequent hospitalisation due to acute arterial cardiovascular events: a 20-year cohort study. Lancet 2007; 370: 1773-1779.

57 McIntyre KM, Sasahara AA. The hemodynamic response to pulmonary embolism in patients without prior cardiopulmonary disease. Am J Cardiol 1971; 28: 288-294.

58 Smulders YM. Pathophysiology and treatment of haemodynamic instability in acute pulmonary embolism: the pivotal role of pulmonary vasoconstriction. Cardiovasc Res 2000; 48: 23-33.

59 Lankhaar JW, Westerhof N, Faes TJC, et al. Quantification of right ventricular afterload in patients with and without pulmonary hypertension. Am J Physiol Heart Circ Physiol 2006; 291: H1731-H1737.

60 Marcus JT, Gan CT, Zwanenburg JJ, et al. Interventricular mechanical asynchrony in pulmonary arterial hypertension: left-to-right delay in peak shortening is related to right ventricular overload and left ventricular underfilling. J Am Coll Cardiol 2008; 51: 750-757.

61 Mauritz GJ, Marcus JT, Westerhof N, et al. Prolonged right ventricular post-systolic isovolumic period in pulmonary arterial hypertension is not a reflection of diastolic dysfunction. Heart 2011; 97: 473-478.

62 Begieneman MP, van de Goot FR, van der Bilt IA, et al. Pulmonary embolism causes endomyocarditis in the human heart. Heart 2008; 94: 450-456.

63 Lankeit M, Jiménez D, Kostrubiec M, et al. Predictive value of the high-sensitivity troponin $\mathrm{T}$ assay and the simplified Pulmonary Embolism Severity Index in hemodynamically stable patients with acute pulmonary embolism: a prospective validation study. Circulation 2011; 124: 2716-2724.

64 Lankeit M, Kempf T, Dellas C, et al. Growth differentiation factor-15 for prognostic assessment of patients with acute pulmonary embolism. Am J Respir Crit Care Med 2008; 177: 1018-1025.

65 Konstantinides SV, Torbicki A, Agnelli G, et al. 2014 ESC Guidelines on the diagnosis and management of acute pulmonary embolism. Eur Heart J 2014; 35: 3033-3073.

66 Burrowes KS, Clark AR, Tawhai MH. Blood flow redistribution and ventilation-perfusion mismatch during embolic pulmonary arterial occlusion. Pulm Circ 2011; 1: 365-376.

67 Konstantinides S, Geibel A, Kasper W, et al. Patent foramen ovale is an important predictor of adverse outcome in patients with major pulmonary embolism. Circulation 1998; 97: 1946-1951.

68 Harjola VP, Mebazaa A, Celutkiene J, et al. Contemporary management of acute right ventricular failure: a statement from the Heart Failure Association and the Working Group on Pulmonary Circulation and Right Ventricular Function of the European Society of Cardiology. Eur J Heart Fail 2016; 18: 226-241.

69 Mebazaa A, Tolppanen H, Mueller C, et al. Acute heart failure and cardiogenic shock: a multidisciplinary practical guidance. Intensive Care Med 2016; 42: 147-163.

70 Thiele H, Ohman EM, Desch S, et al. Management of cardiogenic shock. Eur Heart J 2015; 36: 1223-1230.

71 Righini M, Robert-Ebadi H, Le Gal G. Diagnosis of acute pulmonary embolism. J Thromb Haemost 2017; 15: 1251-1261.

72 Dronkers CEA, van der Hulle T, Le Gal G, et al. Towards a tailored diagnostic standard for future diagnostic studies in pulmonary embolism: communication from the SSC of the ISTH. J Thromb Haemost 2017; 15: 1040-1043.

73 Pollack CV, Schreiber D, Goldhaber SZ, et al. Clinical characteristics, management, and outcomes of patients diagnosed with acute pulmonary embolism in the emergency department: initial report of EMPEROR (Multicenter Emergency Medicine Pulmonary Embolism in the Real World Registry). J Am Coll Cardiol 2011; 57: 700-706.

74 Miniati M, Prediletto R, Formichi B, et al. Accuracy of clinical assessment in the diagnosis of pulmonary embolism. Am J Respir Crit Care Med 1999; 159: 864-871.

75 Wells PS, Ginsberg JS, Anderson DR, et al. Use of a clinical model for safe management of patients with suspected pulmonary embolism. Ann Intern Med 1998; 129: 997-1005.

76 Barco S, Ende-Verhaar YM, Becattini C, et al. Differential impact of syncope on the prognosis of patients with acute pulmonary embolism: a systematic review and meta-analysis. Eur Heart J 2018; 39: 4186-4195.

77 Prandoni P, Lensing AW, Prins $\mathrm{MH}$, et al. Prevalence of pulmonary embolism among patients hospitalized for syncope. N Engl J Med 2016; 375: 1524-1531.

78 Stein PD, Henry JW. Clinical characteristics of patients with acute pulmonary embolism stratified according to their presenting syndromes. Chest 1997; 112: 974-979.

79 White RH. The epidemiology of venous thromboembolism. Circulation 2003; 107: I4-I8.

80 Rodger MA, Carrier M, Jones GN, et al. Diagnostic value of arterial blood gas measurement in suspected pulmonary embolism. Am J Respir Crit Care Med 2000; 162: 2105-2108.

81 Stein PD, Goldhaber SZ, Henry JW, et al. Arterial blood gas analysis in the assessment of suspected acute pulmonary embolism. Chest 1996; 109: 78-81. 
Elliott CG, Goldhaber SZ, Visani L, et al. Chest radiographs in acute pulmonary embolism. Results from the International Cooperative Pulmonary Embolism Registry. Chest 2000; 118: 33-38.

83 Shopp JD, Stewart LK, Emmett TW, et al. Findings from 12-lead electrocardiography that predict circulatory shock from pulmonary embolism: systematic review and meta-analysis. Acad Emerg Med 2015; 22 : 1127-1137.

84 Sanders S, Doust J, Glasziou P. A systematic review of studies comparing diagnostic clinical prediction rules with clinical judgment. PLoS One 2015; 10: e0128233.

85 Penaloza A, Verschuren F, Meyer G, et al. Comparison of the unstructured clinician gestalt, the wells score, and the revised Geneva score to estimate pretest probability for suspected pulmonary embolism. Ann Emerg Med 2013; 62: 117-124 e2

86 Wells PS, Anderson DR, Rodger M, et al. Derivation of a simple clinical model to categorize patients probability of pulmonary embolism: increasing the models utility with the SimpliRED D-dimer. Thromb Haemost 2000; 83: 416-420.

87 Klok FA, Mos IC, Nijkeuter M, et al. Simplification of the revised Geneva score for assessing clinical probability of pulmonary embolism. Arch Intern Med 2008; 168: 2131-2136.

88 Gibson NS, Sohne M, Kruip MJHA, et al. Further validation and simplification of the Wells clinical decision rule in pulmonary embolism. Thromb Haemost 2008; 99: 229-234.

89 Douma RA, Mos IC, Erkens PM, et al. Performance of 4 clinical decision rules in the diagnostic management of acute pulmonary embolism: a prospective cohort study. Ann Intern Med 2011; 154: 709-718.

90 Douma RA, Gibson NS, Gerdes VE, et al. Validity and clinical utility of the simplified Wells rule for assessing clinical probability for the exclusion of pulmonary embolism. Thromb Haemost 2009; 101: 197-200.

91 Le Gal G, Righini M, Roy PM, et al. Prediction of pulmonary embolism in the emergency department: the revised Geneva score. Ann Intern Med 2006; 144: 165-171.

92 Ceriani E, Combescure C, Le Gal G, et al. Clinical prediction rules for pulmonary embolism: a systematic review and meta-analysis. J Thromb Haemost 2010; 8: 957-970.

93 Kline JA, Mitchell AM, Kabrhel C, et al. Clinical criteria to prevent unnecessary diagnostic testing in emergency department patients with suspected pulmonary embolism. J Thromb Haemost 2004; 2: 1247-1255.

94 Penaloza A, Soulie C, Moumneh T, et al. Pulmonary embolism rule-out criteria (PERC) rule in European patients with low implicit clinical probability (PERCEPIC): a multicentre, prospective, observational study. Lancet Haematol 2017; 4: e615-e621.

95 Freund Y, Cachanado M, Aubry A, et al. Effect of the pulmonary embolism rule-out criteria on subsequent thromboembolic events among low-risk emergency department patients: the PROPER randomized clinical trial. JAMA 2018; 319: 559-566.

96 Righini M, Le Gal G, De Lucia S, et al. Clinical usefulness of D-dimer testing in cancer patients with suspected pulmonary embolism. Thromb Haemost 2006; 95: 715-719.

97 Di Nisio M, Sohne M, Kamphuisen PW, et al. D-dimer test in cancer patients with suspected acute pulmonary embolism. J Thromb Haemost 2005; 3: 1239-1242.

98 Miron MJ, Perrier A, Bounameaux H, et al. Contribution of noninvasive evaluation to the diagnosis of pulmonary embolism in hospitalized patients. Eur Respir J 1999; 13: 1365-1370.

99 Chabloz P, Reber G, Boehlen F, et al. TAFI antigen and D-dimer levels during normal pregnancy and at delivery. Br J Haematol 2001; 115: 150-152.

100 Francalanci I, Comeglio P, Liotta AA, et al. D-dimer concentrations during normal pregnancy, as measured by ELISA. Thromb Res 1995; 78: 399-405.

101 Perrier A, Roy PM, Sanchez O, et al. Multidetector-row computed tomography in suspected pulmonary embolism. N Engl J Med 2005; 352: 1760-1768.

102 Perrier A, Roy PM, Aujesky D, et al. Diagnosing pulmonary embolism in outpatients with clinical assessment, D-dimer measurement, venous ultrasound, and helical computed tomography: a multicenter management study. Am J Med 2004; 116: 291-299.

103 Wells PS, Anderson DR, Rodger M, et al. Excluding pulmonary embolism at the bedside without diagnostic imaging: management of patients with suspected pulmonary embolism presenting to the emergency department by using a simple clinical model and D-dimer. Ann Intern Med 2001; 135: 98-107.

104 Carrier M, Righini M, Djurabi RK, et al. VIDAS D-dimer in combination with clinical pre-test probability to rule out pulmonary embolism. A systematic review of management outcome studies. Thromb Haemost 2009; 101: 886-892.

105 Righini M, Goehring C, Bounameaux H, et al. Effects of age on the performance of common diagnostic tests for pulmonary embolism. Am J Med 2000; 109: 357-361.

106 Righini M, Van Es J, Den Exter PL, et al. Age-adjusted D-dimer cutoff levels to rule out pulmonary embolism: the ADJUST-PE study. JAMA 2014; 311: 1117-1124.

107 van der Hulle T, Cheung WY, Kooij S, et al. Simplified diagnostic management of suspected pulmonary embolism (the YEARS study): a prospective, multicentre, cohort study. Lancet 2017; 390: 289-297.

108 Howick J, Cals JW, Jones C, et al. Current and future use of point-of-care tests in primary care: an international survey in Australia, Belgium, The Netherlands, the UK and the USA. BMJ Open 2014; 4: e005611.

109 Kingma AEC, van Stel HF, Oudega R, et al. Multi-faceted implementation strategy to increase use of a clinical guideline for the diagnosis of deep venous thrombosis in primary care. Fam Pract 2017; 34: 446-451.

110 Geersing GJ, Janssen KJ, Oudega R, et al. Excluding venous thromboembolism using point of care D-dimer tests in outpatients: a diagnostic meta-analysis. BMJ 2009; 339: b2990.

111 Geersing GJ, Erkens PM, Lucassen WA, et al. Safe exclusion of pulmonary embolism using the Wells rule and qualitative D-dimer testing in primary care: prospective cohort study. BMJ 2012; 345: e6564.

112 Patel S, Kazerooni EA, Cascade PN. Pulmonary embolism: optimization of small pulmonary artery visualization at multi-detector row CT. Radiology 2003; 227: 455-460.

113 Ghaye B, Szapiro D, Mastora I, et al. Peripheral pulmonary arteries: how far in the lung does multi-detector row spiral CT allow analysis? Radiology 2001; 219: 629-636.

114 Carrier M, Righini M, Wells PS, et al. Subsegmental pulmonary embolism diagnosed by computed tomography: incidence and clinical implications. A systematic review and meta-analysis of the management outcome studies. J Thromb Haemost 2010; 8: 1716-1722. 
115 Stein PD, Fowler SE, Goodman LR, et al. Multidetector computed tomography for acute pulmonary embolism. N Engl J Med 2006; 354: 2317-2327.

116 Reid JH, Coche EE, Inoue T, et al. Is the lung scan alive and well? Facts and controversies in defining the role of lung scintigraphy for the diagnosis of pulmonary embolism in the era of MDCT. Eur J Nucl Med Mol Imaging 2009; 36: 505-521.

117 Waxman $\mathrm{AD}$, Bajc M, Brown $\mathrm{M}$, et al. Appropriate use criteria for ventilation-perfusion imaging in pulmonary embolism: summary and excerpts. J Nucl Med 2017; 58: 13N-15N.

118 Sostman HD, Coleman RE, DeLong DM, et al. Evaluation of revised criteria for ventilation-perfusion scintigraphy in patients with suspected pulmonary embolism. Radiology 1994; 193: 103-107.

119 Gottschalk A, Sostman HD, Coleman RE, et al. Ventilation-perfusion scintigraphy in the PIOPED study. Part II. Evaluation of the scintigraphic criteria and interpretations. J Nucl Med 1993; 34: 1119-1126.

120 Glaser JE, Chamarthy M, Haramati LB, et al. Successful and safe implementation of a trinary interpretation and reporting strategy for V/Q lung scintigraphy. J Nucl Med 2011; 52: 1508-1512.

121 Bajc M, Olsson B, Palmer J, et al. Ventilation/perfusion SPECT for diagnostics of pulmonary embolism in clinical practice. J Intern Med 2008; 264: 379-387.

122 Anderson DR, Kahn SR, Rodger MA, et al. Computed tomographic pulmonary angiography vs ventilation-perfusion lung scanning in patients with suspected pulmonary embolism: a randomized controlled trial. JAMA 2007; 298: 2743-2753.

123 Sostman HD, Stein PD, Gottschalk A, et al. Acute pulmonary embolism: sensitivity and specificity of ventilation-perfusion scintigraphy in PIOPED II study. Radiology 2008; 246: 941-946.

124 Roy PM, Colombet I, Durieux P, et al. Systematic review and meta-analysis of strategies for the diagnosis of suspected pulmonary embolism. BMJ 2005; 331: 259.

125 van Es J, Douma RA, Hezemans RE, et al. Accuracy of X-ray with perfusion scan in young patients with suspected pulmonary embolism. Thromb Res 2015; 136: 221-224.

126 Gutte H, Mortensen J, Jensen CV, et al. Detection of pulmonary embolism with combined ventilation-perfusion SPECT and low-dose CT: head-to-head comparison with multidetector CT angiography. J Nucl Med 2009; 50: 1987-1992.

127 Reinartz P, Wildberger JE, Schaefer W, et al. Tomographic imaging in the diagnosis of pulmonary embolism: a comparison between V/Q lung scintigraphy in SPECT technique and multislice spiral CT. J Nucl Med 2004; 45: 1501-1508.

128 Collart JP, Roelants V, Vanpee D, et al. Is a lung perfusion scan obtained by using single photon emission computed tomography able to improve the radionuclide diagnosis of pulmonary embolism? Nucl Med Commun 2002; 23: 1107-1113.

129 Kumar N, Xie K, Mar W, et al. Software-based hybrid perfusion SPECT/CT provides diagnostic accuracy when other pulmonary embolism imaging is indeterminate. Nucl Med Mol Imaging 2015; 49: 303-311.

130 Ling IT, Naqvi HA, Siew TK, et al. SPECT ventilation perfusion scanning with the addition of low-dose CT for the investigation of suspected pulmonary embolism. Intern Med J 2012; 42: 1257-1261.

131 Le Duc-Pennec A, Le Roux PY, Cornily JC, et al. Diagnostic accuracy of single-photon emission tomography ventilation/perfusion lung scan in the diagnosis of pulmonary embolism. Chest 2012; 141: 381-387.

132 Simanek M, Koranda P. The benefit of personalized hybrid SPECT/CT pulmonary imaging. Am J Nucl Med Mol Imaging 2016; 6: 215-222.

133 Qanadli SD, Hajjam ME, Mesurolle B, et al. Pulmonary embolism detection: prospective evaluation of dual-section helical CT versus selective pulmonary arteriography in 157 patients. Radiology 2000; 217: 447-455.

134 PIOPED Investigators. Value of the ventilation/perfusion scan in acute pulmonary embolism. Results of the prospective investigation of pulmonary embolism diagnosis (PIOPED). JAMA 1990; 263: 2753-2759.

135 Stein PD, Henry JW, Gottschalk A. Reassessment of pulmonary angiography for the diagnosis of pulmonary embolism: relation of interpreter agreement to the order of the involved pulmonary arterial branch. Radiology 1999; 210: 689-691.

136 Diffin DC, Leyendecker JR, Johnson SP, et al. Effect of anatomic distribution of pulmonary emboli on interobserver agreement in the interpretation of pulmonary angiography. AJR Am J Roentgenol 1998; 171: $1085-1089$.

137 Stein PD, Athanasoulis C, Alavi A, et al. Complications and validity of pulmonary angiography in acute pulmonary embolism. Circulation 1992; 85: 462-468.

138 Engelberger RP, Kucher N. Catheter-based reperfusion treatment of pulmonary embolism. Circulation 2011; 124: 2139-2144.

139 Revel MP, Sanchez O, Couchon S, et al. Diagnostic accuracy of magnetic resonance imaging for an acute pulmonary embolism: results of the "IRM-EP" study. J Thromb Haemost 2012; 10: 743-750.

140 Stein PD, Chenevert TL, Fowler SE, et al. Gadolinium-enhanced magnetic resonance angiography for pulmonary embolism: a multicenter prospective study (PIOPED III). Ann Intern Med 2010; 152: 434-443; W142-W143.

141 Leung AN, Bull TM, Jaeschke R, et al. An official American Thoracic Society/Society of Thoracic Radiology clinical practice guideline: evaluation of suspected pulmonary embolism in pregnancy. Am J Respir Crit Care Med 2011; 184: 1200-1208

142 Grifoni S, Olivotto I, Cecchini P, et al. Short-term clinical outcome of patients with acute pulmonary embolism, normal blood pressure, and echocardiographic right ventricular dysfunction. Circulation 2000; 101: 2817-2822.

143 Torbicki A, Kurzyna M, Ciurzynski M, et al. Proximal pulmonary emboli modify right ventricular ejection pattern. Eur Respir J 1999; 13: 616-621.

144 Bova C, Greco F, Misuraca G, et al. Diagnostic utility of echocardiography in patients with suspected pulmonary embolism. Am J Emerg Med 2003; 21: 180-183.

145 Kurnicka K, Lichodziejewska B, Goliszek S, et al. Echocardiographic pattern of acute pulmonary embolism: analysis of 511 consecutive patients. J Am Soc Echocardiogr 2016; 29: 907-913.

146 Kurzyna M, Torbicki A, Pruszczyk P, et al. Disturbed right ventricular ejection pattern as a new Doppler echocardiographic sign of acute pulmonary embolism. Am J Cardiol 2002; 90: 507-511.

147 Casazza F, Bongarzoni A, Capozi A, et al. Regional right ventricular dysfunction in acute pulmonary embolism and right ventricular infarction. Eur J Echocardiogr 2005; 6: 11-14. 
Pruszczyk P, Goliszek S, Lichodziejewska B, et al. Prognostic value of echocardiography in normotensive patients with acute pulmonary embolism. JACC Cardiovasc Imaging 2014; 7: 553-560.

149 Lobo JL, Holley A, Tapson V, et al. Prognostic significance of tricuspid annular displacement in normotensive patients with acute symptomatic pulmonary embolism. J Thromb Haemost 2014; 12: 1020-1027.

150 Platz E, Hassanein AH, Shah A, et al. Regional right ventricular strain pattern in patients with acute pulmonary embolism. Echocardiography 2012; 29: 464-470.

151 Sugiura E, Dohi K, Onishi K, et al. Reversible right ventricular regional non-uniformity quantified by speckle-tracking strain imaging in patients with acute pulmonary thromboembolism. J Am Soc Echocardiogr 2009; 22: 1353-1359.

152 Dresden S, Mitchell P, Rahimi L, et al. Right ventricular dilatation on bedside echocardiography performed by emergency physicians aids in the diagnosis of pulmonary embolism. Ann Emerg Med 2014; 63: 16-24.

153 Casazza F, Becattini C, Guglielmelli E, et al. Prognostic significance of free-floating right heart thromboemboli in acute pulmonary embolism: results from the Italian Pulmonary Embolism Registry. Thromb Haemost 2014; 111: 53-57.

154 Mansencal N, Attias D, Caille V, et al. Computed tomography for the detection of free-floating thrombi in the right heart in acute pulmonary embolism. Eur Radiol 2011; 21: 240-245.

155 Torbicki A, Galié N, Covezzoli A, et al. Right heart thrombi in pulmonary embolism: results from the International Cooperative Pulmonary Embolism Registry. J Am Coll Cardiol 2003; 41: 2245-2251.

156 Casazza F, Bongarzoni A, Centonze F, et al. Prevalence and prognostic significance of right-sided cardiac mobile thrombi in acute massive pulmonary embolism. Am J Cardiol 1997; 79: 1433-1435.

157 Koć M, Kostrubiec M, Elikowski W, et al. Outcome of patients with right heart thrombi: the Right Heart Thrombi European Registry. Eur Respir J 2016; 47: 869-875.

158 Barrios D, Rosa-Salazar V, Morillo R, et al. Prognostic significance of right heart thrombi in patients with acute symptomatic pulmonary embolism: systematic review and meta-analysis. Chest 2017; 151: 409-416.

159 Barrios D, Rosa-Salazar V, Jiménez D, et al. Right heart thrombi in pulmonary embolism. Eur Respir J 2016; 48: $1377-1385$.

160 Guerin L, Couturaud F, Parent F, et al. Prevalence of chronic thromboembolic pulmonary hypertension after acute pulmonary embolism. Prevalence of CTEPH after pulmonary embolism. Thromb Haemost 2014; 112: 598-605.

161 Hull RD, Hirsh J, Carter CJ, et al. Pulmonary angiography, ventilation lung scanning, and venography for clinically suspected pulmonary embolism with abnormal perfusion lung scan. Ann Intern Med 1983; 98: 891-899.

162 Perrier A, Bounameaux H. Ultrasonography of leg veins in patients suspected of having pulmonary embolism. Ann Intern Med 1998; 128: 243-243.

163 Kearon C, Ginsberg JS, Hirsh J. The role of venous ultrasonography in the diagnosis of suspected deep venous thrombosis and pulmonary embolism. Ann Intern Med 1998; 129: 1044-1049.

164 Righini M, Le Gal G, Aujesky D, et al. Diagnosis of pulmonary embolism by multidetector CT alone or combined with venous ultrasonography of the leg: a randomised non-inferiority trial. Lancet 2008; 371: $1343-13452$.

165 Le Gal G, Righini M, Sanchez O, et al. A positive compression ultrasonography of the lower limb veins is highly predictive of pulmonary embolism on computed tomography in suspected patients. Thromb Haemost 2006; 95: 963-966.

166 Da Costa Rodrigues J, Alzuphar S, Combescure C, et al. Diagnostic characteristics of lower limb venous compression ultrasonography in suspected pulmonary embolism: a meta-analysis. J Thromb Haemost 2016; 14: 1765-1772.

167 Nazerian P, Volpicelli G, Gigli C, et al. Diagnostic accuracy of focused cardiac and venous ultrasound examinations in patients with shock and suspected pulmonary embolism. Intern Emerg Med 2018; 13: 567-574.

168 Rademaker J, Griesshaber V, Hidajat N, et al. Combined CT pulmonary angiography and venography for diagnosis of pulmonary embolism and deep vein thrombosis: radiation dose. J Thorac Imaging 2001; 16: 297-299.

169 Kucher N, Luder CM, Dornhofer T, et al. Novel management strategy for patients with suspected pulmonary embolism. Eur Heart J 2003; 24: 366-376.

170 Lucassen W, Geersing GJ, Erkens PM, et al. Clinical decision rules for excluding pulmonary embolism: a meta-analysis. Ann Intern Med 2011; 155: 448-460.

171 van Belle A, Buller HR, Huisman MV, et al. Effectiveness of managing suspected pulmonary embolism using an algorithm combining clinical probability, D-dimer testing, and computed tomography. JAMA 2006; 295: 172-179.

172 Musset D, Parent F, Meyer G, et al. Diagnostic strategy for patients with suspected pulmonary embolism: a prospective multicentre outcome study. Lancet 2002; 360: 1914-1920.

173 Kruip MJHA, Slob MJ, Schijen JHEM, et al. Use of a clinical decision rule in combination with D-dimer concentration in diagnostic workup of patients with suspected pulmonary embolism - A prospective management study. Arch Intern Med 2002; 162: 1631-1635.

174 Perrier A, Desmarais S, Miron MJ, et al. Non-invasive diagnosis of venous thromboembolism in outpatients. Lancet 1999; 353: 190-195.

175 Di Nisio M, Squizzato A, Rutjes AWS, et al. Diagnostic accuracy of D-dimer test for exclusion of venous thromboembolism: a systematic review. J Thromb Haemost 2007; 5: 296-304.

176 Stein PD, Hull RD, Patel KC, et al. D-dimer for the exclusion of acute venous thrombosis and pulmonary embolism: a systematic review. Ann Intern Med 2004; 140: 589-602.

177 Righini M, Le Gal G, Aujesky D, et al. Complete venous ultrasound in outpatients with suspected pulmonary embolism. J Thromb Haemost 2009; 7: 406-412.

178 Aujesky D, Roy PM, Verschuren F, et al. Outpatient versus inpatient treatment for patients with acute pulmonary embolism: an international, open-label, randomised, non-inferiority trial. Lancet 2011; 378: 41-48.

179 Meyer G, Vicaut E, Danays T, et al. Fibrinolysis for patients with intermediate-risk pulmonary embolism. $N$ Engl J Med 2014; 370: 1402-1411.

180 Coutance G, Cauderlier E, Ehtisham J, et al. The prognostic value of markers of right ventricular dysfunction in pulmonary embolism: a meta-analysis. Crit Care 2011; 15: R103. 
181 Sanchez O, Trinquart L, Colombet I, et al. Prognostic value of right ventricular dysfunction in patients with haemodynamically stable pulmonary embolism: a systematic review. Eur Heart J 2008; 29: 1569-1577.

182 Doyen D, Castellani M, Moceri P, et al. Patent foramen ovale and stroke in intermediate-risk pulmonary embolism. Chest 2014; 146: 967-973.

183 Goliszek S, Wisniewska M, Kurnicka K, et al. Patent foramen ovale increases the risk of acute ischemic stroke in patients with acute pulmonary embolism leading to right ventricular dysfunction. Thromb Res 2014; 134: 1052-1056.

184 Becattini C, Agnelli G, Vedovati MC, et al. Multidetector computed tomography for acute pulmonary embolism: diagnosis and risk stratification in a single test. Eur Heart J 2011; 32: 1657-1663.

185 Meinel FG, Nance JW Jr, Schoepf UJ, et al. Predictive value of computed tomography in acute pulmonary embolism: systematic review and meta-analysis. Am J Med 2015; 128: 747-759.e2.

186 Coté B, Jimenez D, Planquette B, et al. Prognostic value of right ventricular dilatation in patients with low-risk pulmonary embolism. Eur Respir J 2017; 50: 1701611.

187 George E, Kumamaru KK, Ghosh N, et al. Computed tomography and echocardiography in patients with acute pulmonary embolism: part 2: prognostic value. J Thorac Imaging 2014; 29: W7-W12.

188 Etesamifard N, Shirani S, Jenab Y, et al. Role of clinical and pulmonary computed tomography angiographic parameters in the prediction of short- and long-term mortality in patients with pulmonary embolism. Intern Emerg Med 2016; 11: 405-413.

189 Aviram G, Soikher E, Bendet A, et al. Prediction of mortality in pulmonary embolism based on left atrial volume measured on CT pulmonary angiography. Chest 2016; 149: 667-675.

190 Aviram G, Sirota-Cohen C, Steinvil A, et al. Automated volumetric analysis of four cardiac chambers in pulmonary embolism: a novel technology for fast risk stratification. Thromb Haemost 2012; 108: 384-393.

191 Kang DK, Thilo C, Schoepf UJ, et al. CT signs of right ventricular dysfunction: prognostic role in acute pulmonary embolism. JACC Cardiovasc Imaging 2011; 4: 841-849.

192 Bach AG, Nansalmaa B, Kranz J, et al. CT pulmonary angiography findings that predict 30-day mortality in patients with acute pulmonary embolism. Eur J Radiol 2015; 84: 332-337.

193 Aviram G, Cohen D, Steinvil A, et al. Significance of reflux of contrast medium into the inferior vena cava on computerized tomographic pulmonary angiogram. Am J Cardiol 2012; 109: 432-437.

194 Bajaj A, Saleeb M, Rathor P, et al. Prognostic value of troponins in acute nonmassive pulmonary embolism: a meta-analysis. Heart Lung 2015; 44: 327-334.

195 Becattini C, Vedovati MC, Agnelli G. Prognostic value of troponins in acute pulmonary embolism: a meta-analysis. Circulation 2007; 116: 427-433.

196 Kaeberich A, Seeber V, Jimenez D, et al. Age-adjusted high-sensitivity troponin T cut-off value for risk stratification of pulmonary embolism. Eur Respir J 2015; 45: 1323-1331.

197 Lankeit M, Friesen D, Aschoff J, et al. Highly sensitive troponin T assay in normotensive patients with acute pulmonary embolism. Eur Heart J 2010; 31: 1836-1844.

198 Boscheri A, Wunderlich C, Langer M, et al. Correlation of heart-type fatty acid-binding protein with mortality and echocardiographic data in patients with pulmonary embolism at intermediate risk. Am Heart J 2010; 160: 294-300.

199 Puls M, Dellas C, Lankeit M, et al. Heart-type fatty acid-binding protein permits early risk stratification of pulmonary embolism. Eur Heart J 2007; 28: 224-229.

200 Dellas C, Puls M, Lankeit M, et al. Elevated heart-type fatty acid-binding protein levels on admission predict an adverse outcome in normotensive patients with acute pulmonary embolism. J Am Coll Cardiol 2010; 55: 2150-2157.

201 Dellas C, Lobo JL, Rivas A, et al. Risk stratification of acute pulmonary embolism based on clinical parameters, H-FABP and multidetector CT. Int J Cardiol 2018; 265: 223-228.

202 Bajaj A, Rathor P, Sehgal V, et al. Risk stratification in acute pulmonary embolism with heart-type fatty acid-binding protein: a meta-analysis. J Crit Care 2015; 30: 1151.e1-7.

203 Henzler T, Roeger S, Meyer M, et al. Pulmonary embolism: CT signs and cardiac biomarkers for predicting right ventricular dysfunction. Eur Respir J 2012; 39: 919-926.

204 Klok FA, Mos IC, Huisman MV. Brain-type natriuretic peptide levels in the prediction of adverse outcome in patients with pulmonary embolism: a systematic review and meta-analysis. Am J Respir Crit Care Med 2008; 178: 425-430.

205 Kucher N, Goldhaber SZ. Cardiac biomarkers for risk stratification of patients with acute pulmonary embolism. Circulation 2003; 108: 2191-2194.

206 Agterof MJ, Schutgens RE, Snijder RJ, et al. Out of hospital treatment of acute pulmonary embolism in patients with a low NT-proBNP level. J Thromb Haemost 2010; 8: 1235-1241.

207 Lankeit M, Jimenez D, Kostrubiec M, et al. Validation of N-terminal pro-brain natriuretic peptide cut-off values for risk stratification of pulmonary embolism. Eur Respir J 2014; 43: 1669-1677.

208 Vanni S, Viviani G, Baioni M, et al. Prognostic value of plasma lactate levels among patients with acute pulmonary embolism: the thrombo-embolism lactate outcome study. Ann Emerg Med 2013; 61: 330-338.

209 Vanni S, Jimenez D, Nazerian P, et al. Short-term clinical outcome of normotensive patients with acute PE and high plasma lactate. Thorax 2015; 70: 333-338.

210 Vanni S, Nazerian P, Bova C, et al. Comparison of clinical scores for identification of patients with pulmonary embolism at intermediate-high risk of adverse clinical outcome: the prognostic role of plasma lactate. Intern Emerg Med 2017; 12: 657-665.

211 Kostrubiec M, Plywaczewska M, Jiménez $\mathrm{D}$, et al. The prognostic value of renal function in acute pulmonary embolism-a multi-centre cohort study. Thromb Haemost 2019; 119: 140-148.

212 Kostrubiec M, Labyk A, Pedowska-Wloszek J, et al. Neutrophil gelatinase-associated lipocalin, cystatin C and eGFR indicate acute kidney injury and predict prognosis of patients with acute pulmonary embolism. Heart 2012; 98: 1221-1228.

213 Zhou XY, Chen HL, Ni SS. Hyponatremia and short-term prognosis of patients with acute pulmonary embolism: a meta-analysis. Int J Cardiol 2017; 227: 251-256.

214 Vuilleumier N, Simona A, Mean M, et al. Comparison of cardiac and non-cardiac biomarkers for risk stratification in elderly patients with non-massive pulmonary embolism. PLoS One 2016; 11: e0155973. 
215 Wyzgal A, Koc M, Pacho S, et al. Plasma copeptin for short term risk stratification in acute pulmonary embolism. J Thromb Thrombolysis 2016; 41: 563-568.

216 Hellenkamp K, Schwung J, Rossmann H, et al. Risk stratification of normotensive pulmonary embolism: prognostic impact of copeptin. Eur Respir J 2015; 46: 1701-1710.

217 Hellenkamp K, Pruszczyk P, Jimenez D, et al. Prognostic impact of copeptin in pulmonary embolism: a multicentre validation study. Eur Respir J 2018; 51: 1702037.

218 Jimenez D, Lobo JL, Fernandez-Golfin C, et al. Effectiveness of prognosticating pulmonary embolism using the ESC algorithm and the Bova score. Thromb Haemost 2016; 115: 827-834.

219 Hobohm L, Hellenkamp K, Hasenfuss G, et al. Comparison of risk assessment strategies for not-high-risk pulmonary embolism. Eur Respir J 2016; 47: 1170-1178.

220 Fernandez C, Bova C, Sanchez O, et al. Validation of a model for identification of patients at intermediate to high risk for complications associated with acute symptomatic pulmonary embolism. Chest 2015; 148: 211-218.

221 Bova C, Sanchez O, Prandoni P, et al. Identification of intermediate-risk patients with acute symptomatic pulmonary embolism. Eur Respir J 2014; 44: 694-703.

222 Dellas C, Tschepe M, Seeber V, et al. A novel H-FABP assay and a fast prognostic score for risk assessment of normotensive pulmonary embolism. Thromb Haemost 2014; 111: 996-1003.

223 Lankeit M, Friesen D, Schafer K, et al. A simple score for rapid risk assessment of non-high-risk pulmonary embolism. Clin Res Cardiol 2013; 102: 73-80.

224 Single-bolus tenecteplase plus heparin compared with heparin alone for normotensive patients with acute pulmonary embolism who have evidence of right ventricular dysfunction and myocardial injury: rationale and design of the Pulmonary Embolism Thrombolysis (PEITHO) trial. Am Heart J 2012; 163: 33-38.

225 Donze J, Le Gal G, Fine MJ, et al. Prospective validation of the Pulmonary Embolism Severity Index. A clinical prognostic model for pulmonary embolism. Thromb Haemost 2008; 100: 943-948.

226 Aujesky D, Obrosky DS, Stone RA, et al. Derivation and validation of a prognostic model for pulmonary embolism. Am J Respir Crit Care Med 2005; 172: 1041-1046.

227 Elias A, Mallett S, Daoud-Elias M, et al. Prognostic models in acute pulmonary embolism: a systematic review and meta-analysis. BMJ Open 2016; 6: e010324.

228 Kohn CG, Mearns ES, Parker MW, et al. Prognostic accuracy of clinical prediction rules for early post-pulmonary embolism all-cause mortality: a bivariate meta-analysis. Chest 2015; 147: 1043-1062.

229 Jiménez D, Aujesky D, Moores L, et al. Simplification of the pulmonary embolism severity index for prognostication in patients with acute symptomatic pulmonary embolism. Arch Intern Med 2010; 170: 1383-1389.

230 Righini M, Roy PM, Meyer G, et al. The Simplified Pulmonary Embolism Severity Index (PESI): validation of a clinical prognostic model for pulmonary embolism. J Thromb Haemost 2011; 9: 2115-2117.

231 Sam A, Sanchez D, Gomez V, et al. The shock index and the simplified PESI for identification of low-risk patients with acute pulmonary embolism. Eur Respir J 2011; 37: 762-766.

232 Jiménez D, Aujesky D, Díaz G, et al. Prognostic significance of deep vein thrombosis in patients presenting with acute symptomatic pulmonary embolism. Am J Respir Crit Care Med 2010; 181: 983-991.

233 Becattini C, Cohen AT, Agnelli G, et al. Risk stratification of patients with acute symptomatic pulmonary embolism based on presence or absence of lower extremity DVT: systematic review and meta-analysis. Chest 2016; 149: 192-200.

234 Barco S, Mahmoudpour SH, Planquette B, et al. Prognostic value of right ventricular dysfunction or elevated cardiac biomarkers in patients with low-risk pulmonary embolism: a systematic review and meta-analysis. Eur Heart J 2019; 40: 902-910.

235 Becattini C, Agnelli G, Lankeit M, et al. Acute pulmonary embolism: mortality prediction by the 2014 European Society of Cardiology risk stratification model. Eur Respir J 2016; 48: 780-786.

236 Messika J, Goutorbe P, Hajage D, et al. Severe pulmonary embolism managed with high-flow nasal cannula oxygen therapy. Eur J Emerg Med 2017; 24: 230-232.

237 Lacroix G, Pons F, D'Aranda E, et al. High-flow oxygen, a therapeutic bridge while awaiting thrombolysis in pulmonary embolism? Am J Emerg Med 2013; 31: 463.e1-463.e2.

238 Mercat A, Diehl JL, Meyer G, et al. Hemodynamic effects of fluid loading in acute massive pulmonary embolism. Crit Care Med 1999; 27: 540-544.

239 Green EM, Givertz MM. Management of acute right ventricular failure in the intensive care unit. Curr Heart Fail Rep 2012; 9: 228-235.

240 Ghignone M, Girling L, Prewitt RM. Volume expansion versus norepinephrine in treatment of a low cardiac output complicating an acute increase in right ventricular afterload in dogs. Anesthesiology 1984; 60: 132-135.

241 Manier G, Castaing Y. Influence of cardiac output on oxygen exchange in acute pulmonary embolism. Am Rev Respir Dis 1992; 145: 130-136.

242 Kerbaul F, Gariboldi V, Giorgi R, et al. Effects of levosimendan on acute pulmonary embolism-induced right ventricular failure. Crit Care Med 2007; 35: 1948-1954.

243 Capellier G, Jacques T, Balvay P, et al. Inhaled nitric oxide in patients with pulmonary embolism. Intensive Care Med 1997; 23: 1089-1092.

244 Szold O, Khoury W, Biderman P, et al. Inhaled nitric oxide improves pulmonary functions following massive pulmonary embolism: a report of four patients and review of the literature. Lung 2006; 184: 1-5.

245 Summerfield DT, Desai H, Levitov A, et al. Inhaled nitric oxide as salvage therapy in massive pulmonary embolism: a case series. Respir Care 2012; 57: 444-448.

246 Bhat T, Neuman A, Tantary M, et al. Inhaled nitric oxide in acute pulmonary embolism: a systematic review. Rev Cardiovasc Med 2015; 16: 1-8.

247 Corsi F, Lebreton G, Brechot $\mathrm{N}$, et al. Life-threatening massive pulmonary embolism rescued by venoarterial-extracorporeal membrane oxygenation. Crit Care 2017; 21: 76.

248 Weinberg A, Tapson VF, Ramzy D. Massive pulmonary embolism: extracorporeal membrane oxygenation and surgical pulmonary embolectomy. Semin Respir Crit Care Med 2017; 38: 66-72.

249 Dolmatova EV, Moazzami K, Cocke TP, et al. Extracorporeal membrane oxygenation in massive pulmonary embolism. Heart Lung 2017; 46: 106-109. 
Swol J, Buchwald D, Strauch J, et al. Extracorporeal life support (ECLS) for cardiopulmonary resuscitation (CPR) with pulmonary embolism in surgical patients - a case series. Perfusion 2016; 31: 54-59.

251 Yusuff HO, Zochios V, Vuylsteke A. Extracorporeal membrane oxygenation in acute massive pulmonary embolism: a systematic review. Perfusion 2015; 30: 611-616.

252 Meneveau N, Guillon B, Planquette B, et al. Outcomes after extracorporeal membrane oxygenation for the treatment of high-risk pulmonary embolism: a multicentre series of 52 cases. Eur Heart J 2018; 39: 4196-4204.

253 Shokr M, Rashed A, Mostafa A, et al. Impella RP support and catheter-directed thrombolysis to treat right ventricular failure caused by pulmonary embolism in 2 patients. Tex Heart Inst J 2018; 45: 182-185.

254 Kumar Bhatia N, Dickert NW, Samady H, et al. The use of hemodynamic support in massive pulmonary embolism. Catheter Cardiovasc Interv 2017; 90: 516-520.

255 Perkins GD, Olasveengen TM, Maconochie I, et al. European Resuscitation Council Guidelines for Resuscitation: 2017 update. Resuscitation 2018; 123: 43-50.

256 Soar J, Nolan JP, Böttiger BW, et al. European Resuscitation Council Guidelines for Resuscitation 2015: Section 3. Adult advanced life support. Resuscitation 2015; 95: 100-147.

257 Truhlář A, Deakin CD, Soar J, et al. European Resuscitation Council Guidelines for Resuscitation 2015: Section 4. Cardiac arrest in special circumstances. Resuscitation 2015; 95: 148-201.

258 Aso S, Matsui H, Fushimi K, et al. In-hospital mortality and successful weaning from venoarterial extracorporeal membrane oxygenation: analysis of 5,263 patients using a national inpatient database in Japan. Crit Care 2016; 20: 80 .

259 Steffel J, Verhamme P, Potpara TS, et al. The 2018 European Heart Rhythm Association Practical Guide on the use of non-vitamin $\mathrm{K}$ antagonist oral anticoagulants in patients with atrial fibrillation. Eur Heart J 2018; 39: 1330-1393.

260 Agnelli G, Buller HR, Cohen A, et al. Oral apixaban for the treatment of acute venous thromboembolism. $N$ Engl J Med 2013; 369: 799-808.

261 Buller HR, Prins MH, Lensin AW, et al. Oral rivaroxaban for the treatment of symptomatic pulmonary embolism. N Engl J Med 2012; 366: 1287-1297.

262 Cossette B, Pelletier ME, Carrier N, et al. Evaluation of bleeding risk in patients exposed to therapeutic unfractionated or low-molecular-weight heparin: a cohort study in the context of a quality improvement initiative. Ann Pharmacother 2010; 44: 994-1002.

263 Erkens PM, Prins MH. Fixed dose subcutaneous low molecular weight heparins versus adjusted dose unfractionated heparin for venous thromboembolism. Cochrane Database Syst Rev 2010; 9: CD001100.

264 Stein PD, Hull RD, Matta F, et al. Incidence of thrombocytopenia in hospitalized patients with venous thromboembolism. Am J Med 2009; 122: 919-930.

265 Prandoni P, Siragusa S, Girolami B, et al. The incidence of heparin-induced thrombocytopenia in medical patients treated with low-molecular-weight heparin: a prospective cohort study. Blood 2005; 106: 3049-3054.

266 Raschke RA, Reilly BM, Guidry JR, et al. The weight-based heparin dosing nomogram compared with a 'standard care" nomogram. A randomized controlled trial. Ann Intern Med 1993; 119: 874-881.

267 van Es N, Coppens M, Schulman S, et al. Direct oral anticoagulants compared with vitamin K antagonists for acute venous thromboembolism: evidence from phase 3 trials. Blood 2014; 124: 1968-1975.

268 van der Hulle T, Kooiman J, den Exter PL, et al. Effectiveness and safety of novel oral anticoagulants as compared with vitamin $\mathrm{K}$ antagonists in the treatment of acute symptomatic venous thromboembolism: a systematic review and meta-analysis. J Thromb Haemost 2014; 12: 320-328.

269 Witt DM, Clark NP, Kaatz S, et al. Guidance for the practical management of warfarin therapy in the treatment of venous thromboembolism. J Thromb Thrombolysis 2016; 41: 187-205.

270 Carlquist JF, Anderson JL. Using pharmacogenetics in real time to guide warfarin initiation: a clinician update. Circulation 2011; 124: 2554-2559.

271 Epstein RS, Moyer TP, Aubert RE, et al. Warfarin genotyping reduces hospitalization rates results from the MM-WES (Medco-Mayo Warfarin Effectiveness study). J Am Coll Cardiol 2010; 55: 2804-2812.

272 Kheiri B, Abdalla A, Haykal T, et al. Meta-analysis of genotype-guided versus standard dosing of vitamin K antagonists. Am J Cardiol 2018; 121: 879-887.

273 Mearns ES, White CM, Kohn CG, et al. Quality of vitamin K antagonist control and outcomes in atrial fibrillation patients: a meta-analysis and meta-regression. Thromb J 2014; 12: 14.

274 Garcia DA, Witt DM, Hylek E, et al. Delivery of optimized anticoagulant therapy: consensus statement from the Anticoagulation Forum. Ann Pharmacother 2008; 42: 979-988.

275 Sharma P, Scotland G, Cruickshank M, et al. Is self-monitoring an effective option for people receiving long-term vitamin $\mathrm{K}$ antagonist therapy? A systematic review and economic evaluation. BMJ Open 2015; 5: e007758.

276 Goldhaber SZ, Come PC, Lee RT, et al. Alteplase versus heparin in acute pulmonary embolism: randomised trial assessing right-ventricular function and pulmonary perfusion. Lancet 1993; 341: 507-511.

277 Dalla-Volta S, Palla A, Santolicandro A, et al. PAIMS 2: alteplase combined with heparin versus heparin in the treatment of acute pulmonary embolism. Plasminogen activator Italian multicenter study 2. J Am Coll Cardiol 1992; 20: 520-526.

278 Kline JA, Nordenholz KE, Courtney DM, et al. Treatment of submassive pulmonary embolism with tenecteplase or placebo: cardiopulmonary outcomes at 3 months: multicenter double-blind, placebo-controlled randomized trial. I Thromb Haemost 2014; 12: 459-468.

279 Becattini C, Agnelli G, Salvi A, et al. Bolus tenecteplase for right ventricle dysfunction in hemodynamically stable patients with pulmonary embolism. Thromb Res 2010; 125: e82-e86.

280 Daniels LB, Parker JA, Patel SR, et al. Relation of duration of symptoms with response to thrombolytic therapy in pulmonary embolism. Am J Cardiol 1997; 80: 184-188.

281 Meneveau N, Seronde MF, Blonde MC, et al. Management of unsuccessful thrombolysis in acute massive pulmonary embolism. Chest 2006; 129: 1043-1050.

282 Marti C, John G, Konstantinides S, et al. Systemic thrombolytic therapy for acute pulmonary embolism: a systematic review and meta-analysis. Eur Heart J 2015; 36: 605-614.

283 Chatterjee S, Chakraborty A, Weinberg I, et al. Thrombolysis for pulmonary embolism and risk of all-cause mortality, major bleeding, and intracranial hemorrhage: a meta-analysis. JAMA 2014; 311: 2414-2421. 
284 Sharifi M, Bay C, Skrocki L, et al. Moderate pulmonary embolism treated with thrombolysis (from the "MOPETT" Trial). Am J Cardiol 2013; 111: 273-277.

285 Wang C, Zhai Z, Yang Y, et al. Efficacy and safety of low dose recombinant tissue-type plasminogen activator for the treatment of acute pulmonary thromboembolism: a randomized, multicenter, controlled trial. Chest 2010; 137: $254-262$

286 Tebbe U, Graf A, Kamke W, et al. Hemodynamic effects of double bolus reteplase versus alteplase infusion in massive pulmonary embolism. Am Heart J 1999; 138: 39-44.

287 Tebbe U, Bramlage P, Graf A, et al. Desmoteplase in acute massive pulmonary thromboembolism. Thromb Haemost 2009; 101: 557-562.

288 Konstantinides SV, Vicaut E, Danays T, et al. Impact of thrombolytic therapy on the long-term outcome of intermediate-risk pulmonary embolism. J Am Coll Cardiol 2017; 69: 1536-1544.

289 Galiè N, Humbert M, Vachiery JL, et al. 2015 ESC/ERS Guidelines for the diagnosis and treatment of pulmonary hypertension: The Joint Task Force for the Diagnosis and Treatment of Pulmonary Hypertension of the European Society of Cardiology (ESC) and the European Respiratory Society (ERS). Eur Heart J 2016; 37: 67-119.

290 Tafur AJ, Shamoun FE, Patel SI, et al. Catheter-directed treatment of pulmonary embolism: a systematic review and meta-analysis of modern literature. Clin Appl Thromb Hemost 2017; 23: 821-829.

291 Kaymaz C, Akbal OY, Tanboga IH, et al. Ultrasound-assisted catheter-directed thrombolysis in high-risk and intermediate-high-risk pulmonary embolism: a meta-analysis. Curr Vasc Pharmacol 2018; 16: 179-189.

292 Bajaj NS, Kalra R, Arora P, et al. Catheter-directed treatment for acute pulmonary embolism: systematic review and single-arm meta-analyses. Int J Cardiol 2016; 225: 128-139.

293 Kucher N, Boekstegers P, Muller OJ, et al. Randomized, controlled trial of ultrasound-assisted catheter-directed thrombolysis for acute intermediate-risk pulmonary embolism. Circulation 2014; 129: 479-486.

294 Piazza G, Hohlfelder B, Jaff MR, et al. A prospective, single-arm, multicenter trial of ultrasound-facilitated, catheter-directed, low-dose fibrinolysis for acute massive and submassive pulmonary embolism: the SEATTLE II study. JACC Cardiovasc Interv 2015; 8: 1382-1392.

295 Tapson VF, Sterling K, Jones N, et al. A randomized trial of the optimum duration of acoustic pulse thrombolysis procedure in acute intermediate-risk pulmonary embolism: the OPTALYSE PE trial. JACC Cardiovasc Interv 2018; 11: 1401-1410.

296 Kuo WT, Banerjee A, Kim PS, et al. Pulmonary Embolism Response to Fragmentation, Embolectomy, and Catheter Thrombolysis (PERFECT): initial results from a prospective multicenter Registry. Chest 2015; 148: 667-673.

297 Lee T, Itagaki S, Chiang YP, et al. Survival and recurrence after acute pulmonary embolism treated with pulmonary embolectomy or thrombolysis in New York State, 1999 to 2013. J Thorac Cardiovasc Surg 2018; 155: 1084-1090.e12.

298 Wu MY, Liu YC, Tseng YH, et al. Pulmonary embolectomy in high-risk acute pulmonary embolism: the effectiveness of a comprehensive therapeutic algorithm including extracorporeal life support. Resuscitation 2013; 84: $1365-1370$

299 Keeling WB, Sundt T, Leacche M, et al. Outcomes after surgical pulmonary embolectomy for acute pulmonary embolus: a multi-institutional study. Ann Thorac Surg 2016; 102: 1498-1502.

300 Pasrija C, Kronfli A, Rouse M, et al. Outcomes after surgical pulmonary embolectomy for acute submassive and massive pulmonary embolism: a single-center experience. J Thorac Cardiovasc Surg 2018; 155: 1095-1106.e2.

301 Dudzinski DM, Piazza G. Multidisciplinary pulmonary embolism response teams. Circulation 2016; 133: 98-103.

302 Mismetti P, Laporte S, Pellerin O, et al. Effect of a retrievable inferior vena cava filter plus anticoagulation vs anticoagulation alone on risk of recurrent pulmonary embolism: a randomized clinical trial. JAMA 2015; 313: $1627-1635$.

303 PREPIC Study Group. Eight-year follow-up of patients with permanent vena cava filters in the prevention of pulmonary embolism: the PREPIC (Prevention du Risque d'Embolie Pulmonaire par Interruption Cave) randomized study. Circulation 2005; 112: 416-422.

304 Decousus H, Leizorovicz A, Parent F, et al. A clinical trial of vena caval filters in the prevention of pulmonary embolism in patients with proximal deep-vein thrombosis. Prevention du Risque d'Embolie Pulmonaire par Interruption Cave Study Group. N Engl J Med 1998; 338: 409-415.

305 Bikdeli B, Chatterjee S, Desai NR, et al. Inferior vena cava filters to prevent pulmonary embolism: systematic review and meta-analysis. J Am Coll Cardiol 2017; 70: 1587-1597.

306 Jia Z, Wu A, Tam M, et al. Caval penetration by inferior vena cava filters: a systematic literature review of clinical significance and management. Circulation 2015; 132: 944-952.

307 Durack JC, Westphalen AC, Kekulawela S, et al. Perforation of the IVC: rule rather than exception after longer indwelling times for the Gunther Tulip and Celect retrievable filters. Cardiovasc Intervent Radiol 2012; 35: 299-308.

308 Angel LF, Tapson V, Galgon RE, et al. Systematic review of the use of retrievable inferior vena cava filters. $J$ Vasc Interv Radiol 2011; 22: 1522-1530.e3.

309 Buller HR, Davidson BL, Decousus H, et al. Fondaparinux or enoxaparin for the initial treatment of symptomatic deep venous thrombosis: a randomized trial. Ann Intern Med 2004; 140: 867-873.

310 Buller HR, Davidson BL, Decousus H, et al. Subcutaneous fondaparinux versus intravenous unfractionated heparin in the initial treatment of pulmonary embolism. N Engl J Med 2003; 349: 1695-1702.

311 Robertson L, Jones LE. Fixed dose subcutaneous low molecular weight heparins versus adjusted dose unfractionated heparin for the initial treatment of venous thromboembolism. Cochrane Database Syst Rev 2017; 2: CD001100.

312 Schulman S, Kakkar AK, Goldhaber SZ, et al. Treatment of acute venous thromboembolism with dabigatran or warfarin and pooled analysis. Circulation 2014; 129: 764-772.

313 Buller HR, Decousus H, Grosso MA, et al. Edoxaban versus warfarin for the treatment of symptomatic venous thromboembolism. N Engl J Med 2013; 369: 1406-1415.

314 Schulman S, Kearon C, Kakkar AK, et al. Dabigatran versus warfarin in the treatment of acute venous thromboembolism. N Engl J Med 2009; 361: 2342-2352. 
315 Brandjes DP, Heijboer H, Buller HR, et al. Acenocoumarol and heparin compared with acenocoumarol alone in the initial treatment of proximal-vein thrombosis. N Engl J Med 1992; 327: 1485-1489.

316 Hull RD, Raskob GE, Rosenbloom D, et al. Heparin for 5 days as compared with 10 days in the initial treatment of proximal venous thrombosis. N Engl J Med 1990; 322: 1260-1264.

317 Zondag W, Mos IC, Creemers-Schild D, et al. Outpatient treatment in patients with acute pulmonary embolism: the Hestia Study. J Thromb Haemost 2011; 9: 1500-1507.

318 den Exter PL, Zondag W, Klok FA, et al. Efficacy and safety of outpatient treatment based on the Hestia clinical decision rule with or without NT-proBNP testing in patients with acute pulmonary embolism: a randomized clinical trial. Am J Respir Crit Care Med 2016; 194: 998-1006.

319 Barco S, Schmidtmann I, Ageno W, et al. Early discharge and home treatment of patients with low-risk pulmonary embolism with the oral factor Xa inhibitor rivaroxaban: an international multicentre single-arm clinical trial. Eur Heart J 2019; in press [https://doi.org/10.1093/eurheartj/ehz367].

320 Kline JA, Webb WB, Jones AE, et al. Impact of a rapid rule-out protocol for pulmonary embolism on the rate of screening, missed cases, and pulmonary vascular imaging in an urban US emergency department. Ann Emerg Med 2004; 44: 490-502.

321 Pierre-Justin G, Pierard LA. Management of mobile right heart thrombi: a prospective series. Int J Cardiol 2005; 99: 381-388.

322 Ferrari E, Benhamou M, Berthier F, et al. Mobile thrombi of the right heart in pulmonary embolism: delayed disappearance after thrombolytic treatment. Chest 2005; 127: 1051-1053.

323 Righini M, Aujesky D, Roy PM, et al. Clinical usefulness of D-dimer depending on clinical probability and cutoff value in outpatients with suspected pulmonary embolism. Arch Intern Med 2004; 164: 2483-2487.

324 Brenner DJ, Hall EJ. Computed tomography--an increasing source of radiation exposure. N Engl J Med 2007; 357: 2277-2284.

325 Perrier A, Miron MJ, Desmarais S, et al. Using clinical evaluation and lung scan to rule out suspected pulmonary embolism: is it a valid option in patients with normal results of lower-limb venous compression ultrasonography? Arch Intern Med 2000; 160: 512-516.

326 Stein PD, Sostman HD, Dalen JE, et al. Controversies in diagnosis of pulmonary embolism. Clin Appl Thromb Hemost 2011; 17: 140-149.

327 Roy PM, Moumneh T, Penaloza A, et al. Outpatient management of pulmonary embolism. Thromb Res 2017; 155: $92-100$.

328 Otero R, Uresandi F, Jimenez D, et al. Home treatment in pulmonary embolism. Thromb Res 2010; 126: e1-e5.

329 Torbicki A. Assessing the severity of acute pulmonary embolism: back to the future? Eur Heart J 2019; 40: 911-913.

330 Couturaud F, Sanchez O, Pernod G, et al. Six months vs extended oral anticoagulation after a first episode of pulmonary embolism: the PADIS-PE randomized clinical trial. JAMA 2015; 314: 31-40.

331 Agnelli G, Prandoni P, Becattini C, et al. Extended oral anticoagulant therapy after a first episode of pulmonary embolism. Ann Intern Med 2003; 139: 19-25.

332 Murin S, Romano PS, White RH. Comparison of outcomes after hospitalization for deep venous thrombosis or pulmonary embolism. Thromb Haemost 2002; 88: 407-414.

333 Carrier M, Le GG, Wells PS, et al. Systematic review: case-fatality rates of recurrent venous thromboembolism and major bleeding events among patients treated for venous thromboembolism. Ann Intern Med 2010; 152: $578-589$.

334 Douketis JD, Gu CS, Schulman S, et al. The risk for fatal pulmonary embolism after discontinuing anticoagulant therapy for venous thromboembolism. Ann Intern Med 2007; 147: 766-774.

335 Campbell IA, Bentley DP, Prescott RJ, et al. Anticoagulation for three versus six months in patients with deep vein thrombosis or pulmonary embolism, or both: randomised trial. BMJ 2007; 334: 674.

336 Kearon C, Gent M, Hirsh J, et al. A comparison of three months of anticoagulation with extended anticoagulation for a first episode of idiopathic venous thromboembolism. N Engl J Med 1999; 340: 901-907.

337 Schulman S, Rhedin AS, Lindmarker P, et al. A comparison of six weeks with six months of oral anticoagulant therapy after a first episode of venous thromboembolism. Duration of Anticoagulation Trial Study Group. N Engl J Med 1995; 332: 1661-1665.

338 Kearon C, Ageno W, Cannegieter SC, et al. Categorization of patients as having provoked or unprovoked venous thromboembolism: guidance from the SSC of ISTH. J Thromb Haemost 2016; 14: 1480-1483.

339 Hutten BA, Prins MH, Gent M, et al. Incidence of recurrent thromboembolic and bleeding complications among patients with venous thromboembolism in relation to both malignancy and achieved international normalized ratio: a retrospective analysis. J Clin Oncol 2000; 18: 3078-3083.

340 Baglin $\mathrm{T}$, Luddington R, Brown $\mathrm{K}$, et al. Incidence of recurrent venous thromboembolism in relation to clinical and thrombophilic risk factors: prospective cohort study. Lancet 2003; 362: 523-526.

341 Iorio A, Kearon C, Filippucci E, et al. Risk of recurrence after a first episode of symptomatic venous thromboembolism provoked by a transient risk factor: a systematic review. Arch Intern Med 2010; 170: $1710-1716$.

342 Devreese KMJ, Ortel TL, Pengo V, et al. Laboratory criteria for antiphospholipid syndrome: communication from the SSC of the ISTH. J Thromb Haemost 2018; 16: 809-813.

343 Rodger MA, Le Gal G, Anderson DR, et al. Validating the HERDOO2 rule to guide treatment duration for women with unprovoked venous thrombosis: multinational prospective cohort management study. $B M J$ 2017; 356: j1065.

344 Ensor J, Riley RD, Moore D, et al. Systematic review of prognostic models for recurrent venous thromboembolism (VTE) post-treatment of first unprovoked VTE. BMJ Open 2016; 6: e011190.

345 Linkins LA, Choi PT, Douketis JD. Clinical impact of bleeding in patients taking oral anticoagulant therapy for venous thromboembolism: a meta-analysis. Ann Intern Med 2003; 139: 893-900.

346 Kakkos SK, Kirkilesis GI, Tsolakis IA. Editor's Choice - efficacy and safety of the new oral anticoagulants dabigatran, rivaroxaban, apixaban, and edoxaban in the treatment and secondary prevention of venous thromboembolism: a systematic review and meta-analysis of phase III trials. Eur J Vasc Endovasc Surg 2014; 48: $565-575$. 
Boutitie F, Pinede L, Schulman S, et al. Influence of preceding length of anticoagulant treatment and initia presentation of venous thromboembolism on risk of recurrence after stopping treatment: analysis of individual participants' data from seven trials. BMJ 2011; 342: d3036.

348 Helmert S, Marten S, Mizera H, et al. Effectiveness and safety of apixaban therapy in daily-care patients with atrial fibrillation: results from the Dresden NOAC Registry. J Thromb Thrombolysis 2017; 44: 169-178.

349 Beyer-Westendorf J, Forster K, Pannach S, et al. Rates, management, and outcome of rivaroxaban bleeding in daily care: results from the Dresden NOAC registry. Blood 2014; 124: 955-962.

350 Schulman S, Kearon C, Kakkar AK, et al. Extended use of dabigatran, warfarin, or placebo in venous thromboembolism. N Engl J Med 2013; 368: 709-718.

351 Bauersachs R, Berkowitz SD, Brenner B, et al. Oral rivaroxaban for symptomatic venous thromboembolism. N Engl J Med 2010; 363: 2499-2510.

352 Weitz JI, Lensing AWA, Prins $\mathrm{MH}$, et al. Rivaroxaban or aspirin for extended treatment of venous thromboembolism. N Engl J Med 2017; 376: 1211-1222.

353 Agnelli G, Buller HR, Cohen A, et al. Apixaban for extended treatment of venous thromboembolism. $N$ Engl $J$ Med 2013; 368: 699-708.

354 Pengo V, Denas G, Zoppellaro G, et al. Rivaroxaban vs warfarin in high-risk patients with antiphospholipid syndrome. Blood 2018; 132: 1365-1371.

355 Brighton TA, Eikelboom JW, Mann K, et al. Low-dose aspirin for preventing recurrent venous thromboembolism. N Engl J Med 2012; 367: 1979-1987.

356 Becattini C, Agnelli G, Schenone A, et al. Aspirin for preventing the recurrence of venous thromboembolism. N Engl J Med 2012; 366: 1959-1967.

357 Andreozzi GM, Bignamini AA, Davì G, et al. Sulodexide for the prevention of recurrent venous thromboembolism: the Sulodexide in Secondary Prevention of Recurrent Deep Vein Thrombosis (SURVET) study: a multicenter, randomized, double-blind, placebo-controlled trial. Circulation 2015; 132: 1891-1897.

358 Schulman S, Granqvist S, Holmström M, et al. The duration of oral anticoagulant therapy after a second episode of venous thromboembolism. The Duration of Anticoagulation Trial Study Group. N Engl J Med 1997; 336: 393-398.

359 Schulman S, Svenungsson E, Granqvist S. Anticardiolipin antibodies predict early recurrence of thromboembolism and death among patients with venous thromboembolism following anticoagulant therapy. Duration of Anticoagulation Study Group. Am J Med 1998; 104: 332-338.

360 Lee AY, Kamphuisen PW, Meyer G, et al. Tinzaparin vs warfarin for treatment of acute venous thromboembolism in patients with active cancer: a randomized clinical trial. JAMA 2015; 314: 677-686.

361 Deitcher SR, Kessler CM, Merli G, et al. Secondary prevention of venous thromboembolic events in patients with active cancer: enoxaparin alone versus initial enoxaparin followed by warfarin for a 180-day period. Clin Appl Thromb Hemost 2006; 12: 389-396.

362 Lee AY, Levine MN, Baker RI, et al. Low-molecular-weight heparin versus a coumarin for the prevention of recurrent venous thromboembolism in patients with cancer. $N$ Engl J Med 2003; 349: 146-153.

363 Meyer G, Marjanovic Z, Valcke J, et al. Comparison of low-molecular-weight heparin and warfarin for the secondary prevention of venous thromboembolism in patients with cancer: a randomized controlled study. Arch Intern Med 2002; 162: 1729-1735.

364 Hull RD, Pineo GF, Brant RF, et al. Long-term low-molecular-weight heparin versus usual care in proximal-vein thrombosis patients with cancer. Am J Med 2006; 119: 1062-1072.

365 Posch F, Konigsbrugge O, Zielinski C, et al. Treatment of venous thromboembolism in patients with cancer: a network meta-analysis comparing efficacy and safety of anticoagulants. Thromb Res 2015; 136: 582-589.

366 Raskob GE, van Es N, Verhamme P, et al. Edoxaban for the treatment of cancer-associated venous thromboembolism. N Engl J Med 2018; 378: 615-624.

367 Young AM, Marshall A, Thirlwall J, et al. Comparison of an oral factor Xa inhibitor with low molecular weight heparin in patients with cancer with venous thromboembolism: results of a randomized trial (SELECT-D). J Clin Oncol 2018; 36: 2017-2023.

368 Louzada ML, Carrier M, Lazo-Langner A, et al. Development of a clinical prediction rule for risk stratification of recurrent venous thromboembolism in patients with cancer-associated venous thromboembolism. Circulation 2012; 126: 448-454.

369 Jara-Palomares L, Solier-Lopez A, Elias-Hernandez T, et al. Tinzaparin in cancer associated thrombosis beyond 6months: TiCAT study. Thromb Res 2017; 157: 90-96.

370 Francis CW, Kessler CM, Goldhaber SZ, et al. Treatment of venous thromboembolism in cancer patients with dalteparin for up to 12 months: the DALTECAN study. J Thromb Haemost 2015; 13: 1028-1035.

371 van Es N, Le Gal G, Otten HM, et al. Screening for occult cancer in patients with unprovoked venous thromboembolism: a systematic review and meta-analysis of individual patient data. Ann Intern Med 2017; 167: $410-417$.

372 Carrier M, Lazo-Langner A, Shivakumar S, et al. Screening for occult cancer in unprovoked venous thromboembolism. N Engl J Med 2015; 373: 697-704.

373 Robin P, Le Roux PY, Planquette B, et al. Limited screening with versus without (18)F-fluorodeoxyglucose PET/ CT for occult malignancy in unprovoked venous thromboembolism: an open-label randomised controlled trial. Lancet Oncol 2016; 17: 193-199.

374 Farge D, Bounameaux H, Brenner B, et al. International clinical practice guidelines including guidance for direct oral anticoagulants in the treatment and prophylaxis of venous thromboembolism in patients with cancer. Lancet Oncol 2016; 17: e452-e466.

375 Mandalà M, Falanga A, Roila F. ESMO Guidelines Working Group. Management of venous thromboembolism (VTE) in cancer patients: ESMO Clinical Practice Guidelines. Ann Oncol 2011; 22: vi85-vi92.

376 den Exter PL, Hooijer J, Dekkers OM, et al. Risk of recurrent venous thromboembolism and mortality in patients with cancer incidentally diagnosed with pulmonary embolism: a comparison with symptomatic patients. $J$ Clin Oncol 2011; 29: 2405-2409.

377 Dentali F, Ageno W, Becattini C, et al. Prevalence and clinical history of incidental, asymptomatic pulmonary embolism: a meta-analysis. Thromb Res 2010; 125: 518-522. 
41

413

Napolitano M, Saccullo G, Malato A, et al. Optimal duration of low molecular weight heparin for the treatment of cancer-related deep vein thrombosis: the Cancer-DACUS Study. J Clin Oncol 2014; 32: 3607-3612.

Berg CJ, Callaghan WM, Syverson C, et al. Pregnancy-related mortality in the United States, 1998 to 2005. Obstet Gynecol 2010; 116: 1302-1309.

Heit JA, Kobbervig CE, James AH, et al. Trends in the incidence of venous thromboembolism during pregnancy or postpartum: a 30-year population-based study. Ann Intern Med 2005; 143: 697-706.

Sultan AA, West J, Tata LJ, et al. Risk of first venous thromboembolism in and around pregnancy: a population-based cohort study. Br J Haematol 2012; 156: 366-373.

Henriksson $\mathrm{P}$, Westerlund $\mathrm{E}$, Wallen $\mathrm{H}$, et al. Incidence of pulmonary and venous thromboembolism in pregnancies after in vitro fertilisation: cross sectional study. BMJ 2013; 346: e8632.

Sultan AA, West J, Grainge MJ, et al. Development and validation of risk prediction model for venous thromboembolism in postpartum women: multinational cohort study. BMJ 2016; 355: i6253.

Regitz-Zagrosek V, Roos-Hesselink JW, Bauersachs J, et al. 2018 ESC Guidelines for the management of cardiovascular diseases during pregnancy. Eur Heart J 2018; 39: 3165-3241.

Sheen JJ, Haramati LB, Natenzon A, et al. Performance of low-dose perfusion scintigraphy and CT pulmonary angiography for pulmonary embolism in pregnancy. Chest 2018; 153: 152-160.

van Mens TE, Scheres LJ, de Jong PG, et al. Imaging for the exclusion of pulmonary embolism in pregnancy. Cochrane Database Syst Rev 2017; 1: CD011053.

Hamilton EJ, Green AQ, Cook JA, et al. Investigating for pulmonary embolism in pregnancy: Five year retrospective review of referrals to the acute medical unit of a large teaching hospital. Acute Med 2016; 15: 58-62. Righini M, Robert-Ebadi H, Elias A, et al. Diagnosis of pulmonary embolism during pregnancy: a multicenter prospective management outcome study. Ann Intern Med 2018; 169: 766-773.

Murphy N, Broadhurst DI, Khashan AS, et al. Gestation-specific D-dimer reference ranges: a cross-sectional study. BJOG 2015; 122: 395-400.

Ercan S, Ozkan S, Yucel N, et al. Establishing reference intervals for D-dimer to trimesters. J Matern Fetal Neonatal Med 2015; 28: 983-987.

van der Pol LM, Tromeur C, Bistervels IM, et al. Pregnancy-adapted YEARS algorithm for diagnosis of suspected pulmonary embolism. N Engl J Med 2019; 380: 1139-1149.

Mitchell DP, Rowan M, Loughman E, et al. Contrast monitoring techniques in CT pulmonary angiography: an important and underappreciated contributor to breast dose. Eur J Radiol 2017; 86: 184-189.

Shahir K, McCrea JM, Lozano LA, et al. Reduced z-axis technique for CT pulmonary angiography in pregnancy--validation for practical use and dose reduction. Emerg Radiol 2015; 22: 651-656.

Perisinakis K, Seimenis I, Tzedakis A, et al. Perfusion scintigraphy versus 256-slice CT angiography in pregnant patients suspected of pulmonary embolism: comparison of radiation risks. J Nucl Med 2014; 55: 1273-1280.

Astani SA, Davis LC, Harkness BA, et al. Detection of pulmonary embolism during pregnancy: comparing radiation doses of CTPA and pulmonary scintigraphy. Nucl Med Commun 2014; 35: 704-711.

Chunilal SD, Bates SM. Venous thromboembolism in pregnancy: diagnosis, management and prevention. Thromb Haemost 2009; 101: 428-438.

Bajc M, Neilly JB, Miniati M, et al. EANM guidelines for ventilation/perfusion scintigraphy: Part 1. Pulmonary imaging with ventilation/perfusion single photon emission tomography. Eur J Nucl Med Mol Imaging 2009; 36: 1356-1370.

Nijkeuter M, Geleijns J, De Roos A, et al. Diagnosing pulmonary embolism in pregnancy: rationalizing fetal radiation exposure in radiological procedures. J Thromb Haemost 2004; 2: 1857-1858.

Tromeur C, van der Pol LM, Klok FA, et al. Pitfalls in the diagnostic management of pulmonary embolism in pregnancy. Thromb Res 2017; 151: S86-S91.

Ginsberg JS, Hirsh J, Rainbow AJ, et al. Risks to the fetus of radiologic procedures used in the diagnosis of maternal venous thromboembolic disease. Thromb Haemost 1989; 61: 189-196.

Schembri GP, Miller AE, Smart R. Radiation dosimetry and safety issues in the investigation of pulmonary embolism. Semin Nucl Med 2010; 40: 442-454.

Ramsay R, Byrd L, Tower C, et al. The problem of pulmonary embolism diagnosis in pregnancy. Br J Haematol 2015; 170: 727-728.

Bourjeily G, Khalil H, Raker C, et al. Outcomes of negative multidetector computed tomography with pulmonary angiography in pregnant women suspected of pulmonary embolism. Lung 2012; 190: 105-111.

Chan WS, Ray JG, Murray S, et al. Suspected pulmonary embolism in pregnancy: clinical presentation, results of lung scanning, and subsequent maternal and pediatric outcomes. Arch Intern Med 2002; 162: 1170-1175.

Siegel Y, Kuker R, Banks J, et al. CT pulmonary angiogram quality comparison between early and later pregnancy. Emerg Radiol 2017; 24: 635-640.

Armstrong L, Gleeson F, Mackillop L, et al. Survey of UK imaging practice for the investigation of pulmonary embolism in pregnancy. Clin Radiol 2017; 72: 696-701.

Bajc M, Olsson B, Gottsater A, et al. V/P SPECT as a diagnostic tool for pregnant women with suspected pulmonary embolism. Eur J Nucl Med Mol Imaging 2015; 42: 1325-1330.

Romualdi E, Dentali F, Rancan E, et al. Anticoagulant therapy for venous thromboembolism during pregnancy: a systematic review and a meta-analysis of the literature. J Thromb Haemost 2013; 11: 270-281.

409 Bates SM, Greer IA, Middeldorp S, et al. VTE, thrombophilia, antithrombotic therapy, and pregnancy: Antithrombotic Therapy and Prevention of Thrombosis, 9th ed: American College of Chest Physicians Evidence-Based Clinical Practice Guidelines. Chest 2012; 141: e691S-e736S.

410 Greer IA, Nelson-Piercy C. Low-molecular-weight heparins for thromboprophylaxis and treatment of venous thromboembolism in pregnancy: a systematic review of safety and efficacy. Blood 2005; 106: 401-407.

11 Ainle F N, Wong A, Appleby N, et al. Efficacy and safety of once daily low molecular weight heparin (tinzaparin sodium) in high risk pregnancy. Blood Coagul Fibrinolysis 2008; 19: 689-692.

2 Baglin T, Barrowcliffe TW, Cohen A, et al. Guidelines on the use and monitoring of heparin. Br J Haematol 2006; 133: 19-34.

413 Harenberg J. Is laboratory monitoring of low-molecular-weight heparin therapy necessary? Yes. J Thromb Haemost 2004; 2: 547-550. 
Greer I, Hunt BJ. Low molecular weight heparin in pregnancy: current issues. Br J Haematol 2005; 128: 593-601. Galambosi P, Hiilesmaa V, Ulander VM, et al. Prolonged low-molecular-weight heparin use during pregnancy and subsequent bone mineral density. Thromb Res 2016; 143: 122-126.

Dempfle CE. Minor transplacental passage of fondaparinux in vivo. N Engl J Med 2004; 350: 1914-1915.

Cohen H, Arachchillage DR, Middeldorp S, et al. Management of direct oral anticoagulants in women of childbearing potential: guidance from the SSC of the ISTH. J Thromb Haemost 2016; 14: 1673-1676.

Leffert L, Butwick A, Carvalho B, et al. The Society for Obstetric Anesthesia and Perinatology Consensus Statement on the anesthetic management of pregnant and postpartum women receiving thromboprophylaxis or higher dose anticoagulants. Anesth Analg 2018; 126: 928-944.

Leffert LR, Dubois HM, Butwick AJ, et al. Neuraxial anesthesia in obstetric patients receiving thromboprophylaxis with unfractionated or low-molecular-weight heparin: a systematic review of spinal epidural hematoma. Anesth Analg 2017; 125: 223-231.

Gogarten W, Vandermeulen E, Van Aken $\mathrm{H}$, et al. Regional anaesthesia and antithrombotic agents: recommendations of the European Society of Anaesthesiology. Eur J Anaesthesiol 2010; 27: 999-1015.

Martillotti G, Boehlen F, Robert-Ebadi H, et al. Treatment options for severe pulmonary embolism during pregnancy and the postpartum period: a systematic review. J Thromb Haemost 2017; 15: 1942-1950.

Fitzpatrick KE, Tuffnell D, Kurinczuk JJ, et al. Incidence, risk factors, management and outcomes of amniotic-fluid embolism: a population-based cohort and nested case-control study. BJOG 2016; 123: 100-109.

Society for Maternal-Fetal Medicine, Pacheco LD, Saade G, et al. Amniotic fluid embolism: diagnosis and management. Am J Obstet Gynecol 2016; 215: B16-B24.

Clark SL, Romero R, Dildy GA, et al. Proposed diagnostic criteria for the case definition of amniotic fluid embolism in research studies. Am J Obstet Gynecol 2016; 215: 408-412.

Fong A, Chau CT, Pan D, et al. Amniotic fluid embolism: antepartum, intrapartum and demographic factors. J Matern Fetal Neonatal Med 2015; 28: 793-798.

McDonnell N, Knight M, Peek MJ, et al. Amniotic fluid embolism: an Australian-New Zealand population-based study. BMC Pregnancy Childbirth 2015; 15: 352.

den Exter PL, Van EJ, Kroft LJ, et al. Thromboembolic resolution assessed by CT pulmonary angiography after treatment for acute pulmonary embolism. Thromb Haemost 2015; 114: 26-34.

Klok FA, van der Hulle T, den Exter PL, et al. The post-PE syndrome: a new concept for chronic complications of pulmonary embolism. Blood Rev 2014; 28: 221-226.

Klok FA, van Kralingen KW, van Dijk AP, et al. Prevalence and potential determinants of exertional dyspnea after acute pulmonary embolism. Respir Med 2010; 104: 1744-1749.

Kline JA, Steuerwald MT, Marchick MR, et al. Prospective evaluation of right ventricular function and functiona status 6 months after acute submassive pulmonary embolism: frequency of persistent or subsequent elevation in estimated pulmonary artery pressure. Chest 2009; 136: 1202-1210.

Becattini C, Agnelli G, Pesavento R, et al. Incidence of chronic thromboembolic pulmonary hypertension after a first episode of pulmonary embolism. Chest 2006; 130: 172-175.

Sanchez O, Helley D, Couchon S, et al. Perfusion defects after pulmonary embolism: risk factors and clinical significance. J Thromb Haemost 2010; 8: 1248-1255.

Stevinson BG, Hernandez-Nino J, Rose G, et al. Echocardiographic and functional cardiopulmonary problems 6 months after first-time pulmonary embolism in previously healthy patients. Eur Heart $J$ 2007; 28: $2517-2524$.

4 Meneveau N, Ider O, Seronde MF, et al. Long-term prognostic value of residual pulmonary vascular obstruction at discharge in patients with intermediate- to high-risk pulmonary embolism. Eur Heart J 2013; 34: 693-701.

Kahn SR, Hirsch AM, Akaberi A, et al. Functional and exercise limitations after a first episode of pulmonary embolism: results of the ELOPE prospective cohort study. Chest 2017; 151: 1058-1068.

Kahn SR, Akaberi A, Granton JT, et al. Quality of life, dyspnea, and functional exercise capacity following a first episode of pulmonary embolism: results of the ELOPE cohort study. Am J Med 2017; 130: 990.e9-990.e21.

Albaghdadi MS, Dudzinski DM, Giordano N, et al. Cardiopulmonary exercise testing in patients following massive and submassive pulmonary embolism. J Am Heart Assoc 2018; 7: e006841.

Konstantinides SV, Barco S, Rosenkranz S, et al. Late outcomes after acute pulmonary embolism: rationale and design of FOCUS, a prospective observational multicenter cohort study. J Thromb Thrombolysis 2016; 42: 600-609.

Simonneau G, Hoeper MM. Evaluation of the incidence of rare diseases: difficulties and uncertainties, the example of chronic thromboembolic pulmonary hypertension. Eur Respir J 2017; 49: 1602522.

Ende-Verhaar YM, Cannegieter SC, Vonk Noordegraaf A, et al. Incidence of chronic thromboembolic pulmonary hypertension after acute pulmonary embolism: a contemporary view of the published literature. Eur Respir J 2017; 49: 1601792.

Coquoz N, Weilenmann D, Stolz D, et al. Multicentre observational screening survey for the detection of CTEPH following pulmonary embolism. Eur Respir J 2018; 51: 1702505.

Kramm T, Wilkens H, Fuge J, et al. Incidence and characteristics of chronic thromboembolic pulmonary hypertension in Germany. Clin Res Cardiol 2018; 107: 548-553.

Dorfmuller P, Gunther S, Ghigna MR, et al. Microvascular disease in chronic thromboembolic pulmonary hypertension: a role for pulmonary veins and systemic vasculature. Eur Respir J 2014; 44: 1275-1288.

Azarian R, Wartski M, Collignon MA, et al. Lung perfusion scans and hemodynamics in acute and chronic pulmonary embolism. J Nucl Med 1997; 38: 980-983.

Lewczuk J, Piszko P, Jagas J, et al. Prognostic factors in medically treated patients with chronic pulmonary embolism. Chest 2001; 119: 818-823.

Riedel M, Stanek V, Widimsky J, et al. Longterm follow-up of patients with pulmonary thromboembolism. Late prognosis and evolution of hemodynamic and respiratory data. Chest 1982; 81: 151-158.

Pepke-Zaba J, Delcroix M, Lang I, et al. Chronic thromboembolic pulmonary hypertension (CTEPH): results from an international prospective registry. Circulation 2011; 124: 1973-1981.

ang IM, Simonneau G, Pepke-Zaba JW, et al. Factors associated with diagnosis and operability of chronic thromboembolic pulmonary hypertension. A case-control study. Thromb Haemost 2013; 110: 83-91. 
449 Bonderman D, Wilkens H, Wakounig S, et al. Risk factors for chronic thromboembolic pulmonary hypertension. Eur Respir J 2009; 33: 325-331.

450 Klok FA, Dzikowska-Diduch O, Kostrubiec M, et al. Derivation of a clinical prediction score for chronic thromboembolic pulmonary hypertension after acute pulmonary embolism. J Thromb Haemost 2016; 14: 121-128.

451 Klok FA, Surie S, Kempf T, et al. A simple non-invasive diagnostic algorithm for ruling out chronic thromboembolic pulmonary hypertension in patients after acute pulmonary embolism. Thromb Res 2011; 128: 21-26.

452 Klok FA, Tesche C, Rappold L, et al. External validation of a simple non-invasive algorithm to rule out chronic thromboembolic pulmonary hypertension after acute pulmonary embolism. Thromb Res 2015; 135: 796-801.

453 Pepke-Zaba J, Jansa P, Kim NH, et al. Chronic thromboembolic pulmonary hypertension: role of medical therapy. Eur Respir J 2013; 41: 985-990.

454 Taboada D, Pepke-Zaba J, Jenkins DP, et al. Outcome of pulmonary endarterectomy in symptomatic chronic thromboembolic disease. Eur Respir J 2014; 44: 1635-1645.

455 Tunariu N, Gibbs SJ, Win Z, et al. Ventilation-perfusion scintigraphy is more sensitive than multidetector CTPA in detecting chronic thromboembolic pulmonary disease as a treatable cause of pulmonary hypertension. $J$ Nucl Med 2007; 48: 680-684.

456 Fang W, Ni XH, He JG, et al. Value of radionuclide lung scintigraphy in the diagnosis and quantitative analysis of chronic thromboembolic pulmonary hypertension [article in Chinese]. Zhonghua Xin Xue Guan Bing Za Zhi 2008; 36: 7-10.

457 Ley S, Ley-Zaporozhan J, Pitton MB, et al. Diagnostic performance of state-of-the-art imaging techniques for morphological assessment of vascular abnormalities in patients with chronic thromboembolic pulmonary hypertension (CTEPH). Eur Radiol 2012; 22: 607-616.

458 Fukuda T, Ogo T, Nakanishi N, et al. Evaluation of organized thrombus in distal pulmonary arteries in patients with chronic thromboembolic pulmonary hypertension using cone-beam computed tomography. Jpn J Radiol 2016; 34: 423-431.

459 Shure D, Gregoratos G, Moser KM. Fiberoptic angioscopy: role in the diagnosis of chronic pulmonary arterial obstruction. Ann Intern Med 1985; 103: 844-850.

460 Vuylsteke A, Sharples L, Charman G, et al. Circulatory arrest versus cerebral perfusion during pulmonary endarterectomy surgery (PEACOG): a randomised controlled trial. Lancet 2011; 378: 1379-1387.

461 Jenkins D, Madani M, Fadel E, et al. Pulmonary endarterectomy in the management of chronic thromboembolic pulmonary hypertension. Eur Respir Rev 2017; 26: 160111.

462 Mayer E, Jenkins D, Lindner J, et al. Surgical management and outcome of patients with chronic thromboembolic pulmonary hypertension: results from an international prospective registry. $J$ Thorac Cardiovasc Surg 2011; 141: 702-710.

463 Madani MM, Auger WR, Pretorius V, et al. Pulmonary endarterectomy: recent changes in a single institution's experience of more than 2,700 patients. Ann Thorac Surg 2012; 94: 97-103.

464 Jenkins D, Mayer E, Screaton N, et al. State-of-the-art chronic thromboembolic pulmonary hypertension diagnosis and management. Eur Respir Rev 2012; 21: 32-39.

465 Delcroix M, Lang I, Pepke-Zaba J, et al. Long-term outcome of patients with chronic thromboembolic pulmonary hypertension: results from an international prospective registry. Circulation 2016; 133: 859-871.

466 Cannon JE, Su L, Kiely DG, et al. Dynamic risk stratification of patient long-term outcome after pulmonary endarterectomy: results from the United Kingdom National Cohort. Circulation 2016; 133: 1761-1771.

467 Ogawa A, Satoh T, Fukuda T, et al. Balloon pulmonary angioplasty for chronic thromboembolic pulmonary hypertension: results of a multicenter registry. Circ Cardiovasc Qual Outcomes 2017; 10: e004029.

468 Collaud S, Brenot P, Mercier O, et al. Rescue balloon pulmonary angioplasty for early failure of pulmonary endarterectomy: the earlier the better? Int J Cardiol 2016; 222: 39-40.

469 Ghofrani HA, D'Armini AM, Grimminger F, et al. Riociguat for the treatment of chronic thromboembolic pulmonary hypertension. N Engl J Med 2013; 369: 319-329.

470 Ghofrani HA, Simonneau G, D'Armini AM, et al. Macitentan for the treatment of inoperable chronic thromboembolic pulmonary hypertension (MERIT-1): results from the multicentre, phase 2, randomised, double-blind, placebo-controlled study. Lancet Respir Med 2017; 5: 785-794.

471 Jais X, D'Armini AM, Jansa P, et al. Bosentan for treatment of inoperable chronic thromboembolic pulmonary hypertension: BENEFiT (Bosentan Effects in iNopErable Forms of chronIc Thromboembolic pulmonary hypertension), a randomized, placebo-controlled trial. J Am Coll Cardiol 2008; 52: 2127-2134.

472 van Kan C, van der Plas MN, Reesink HJ, et al. Hemodynamic and ventilatory responses during exercise in chronic thromboembolic disease. J Thorac Cardiovasc Surg 2016; 152: 763-771.

473 Al-Mallah MH, Farah I, Al-Madani W, et al. The impact of nurse-led clinics on the mortality and morbidity of patients with cardiovascular diseases: a systematic review and meta-analysis. J Cardiovasc Nurs 2016; 31: 89-95.

474 Martínez-González NA, Tandjung R, Djalali S, et al. Effects of physician-nurse substitution on clinical parameters: a systematic review and meta-analysis. PLoS One 2014; 9: e89181.

475 Massimi A, De Vito C, Brufola I, et al. Are community-based nurse-led self-management support interventions effective in chronic patients? Results of a systematic review and meta-analysis. PLoS One 2017; 12: e0173617.

476 Ivarsson B, Radegran G, Hesselstrand R, et al. Coping, social support and information in patients with pulmonary arterial hypertension or chronic thromboembolic pulmonary hypertension: a 2-year retrospective cohort study. SAGE Open Med 2018; 6: 2050312117749159.

477 Kim NH, Delcroix M, Jenkins DP, et al. Chronic thromboembolic pulmonary hypertension. J Am Coll Cardiol 2013; 62: D92-D99.

478 Barco S, Russo M, Vicaut E, et al. Incomplete echocardiographic recovery at 6 months predicts long-term sequelae after intermediate-risk pulmonary embolism. A post-hoc analysis of the Pulmonary Embolism Thrombolysis (PEITHO) trial. Clin Res Cardiol 2019; 108: 772-778. 